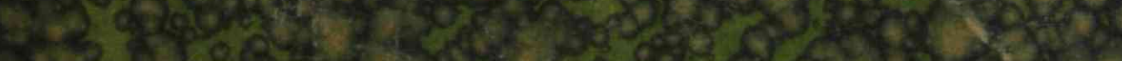

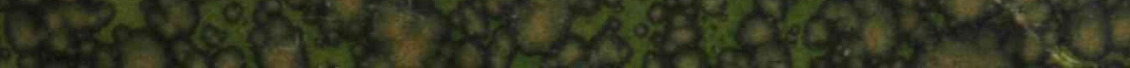

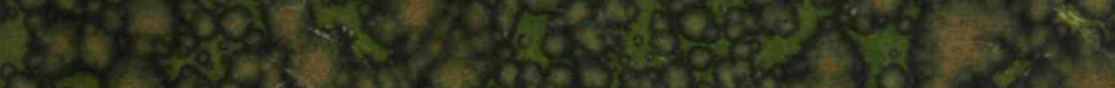

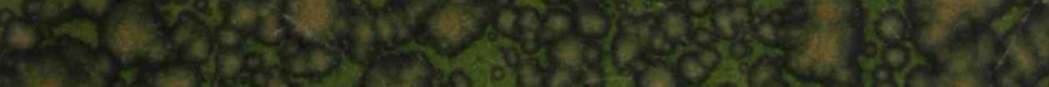

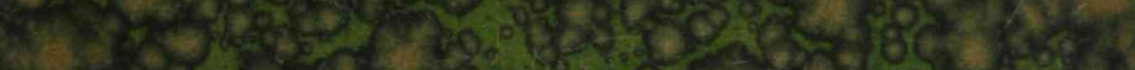

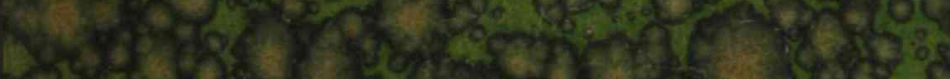

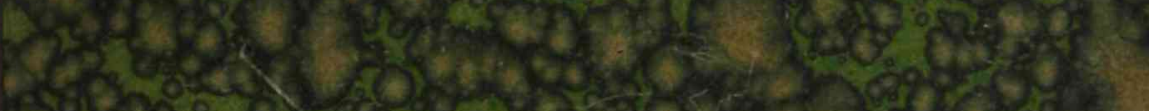

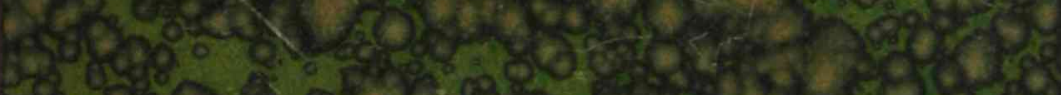

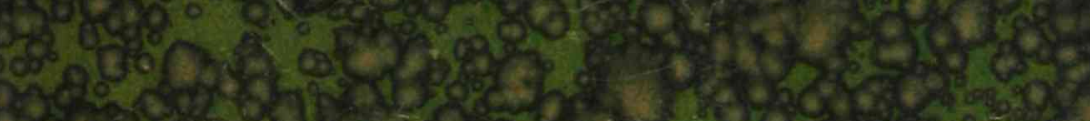

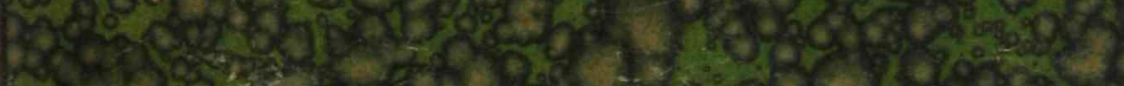

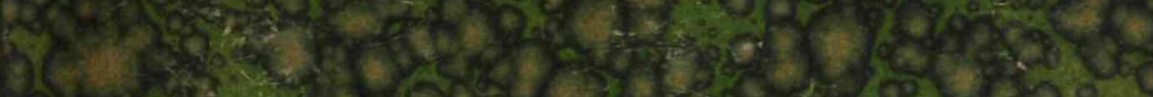

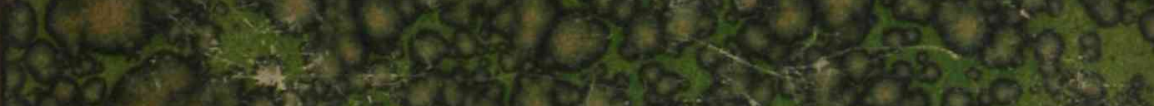

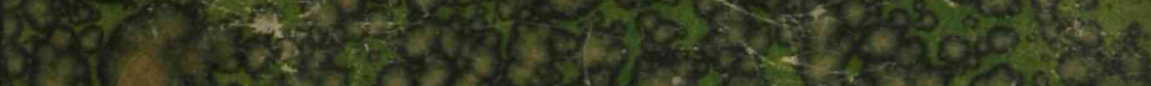

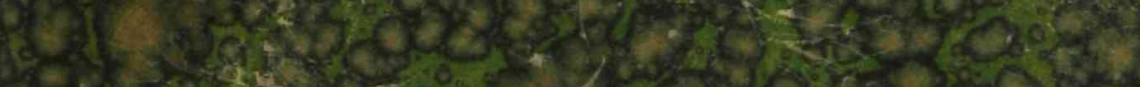

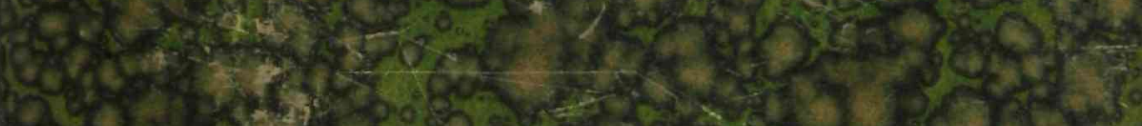
M.9.

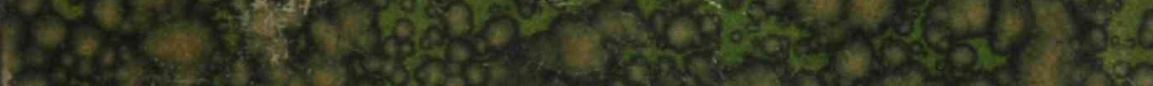

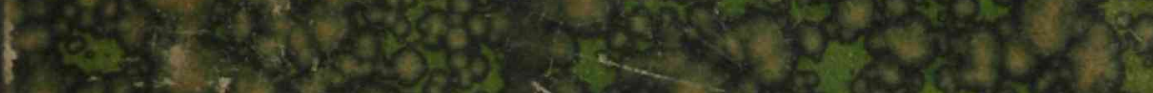

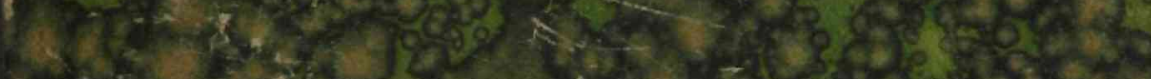
SW

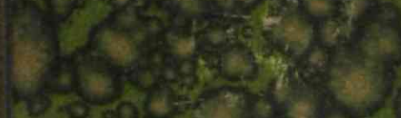

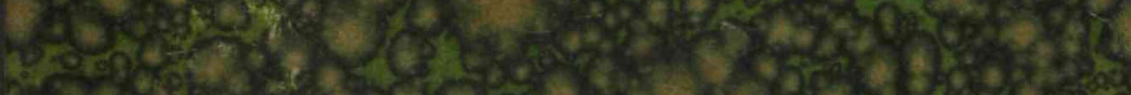

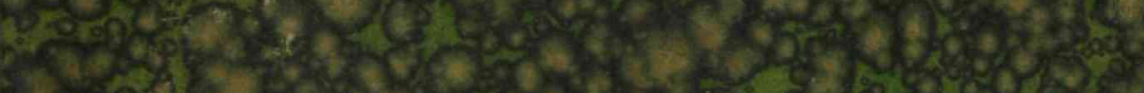
4050 .

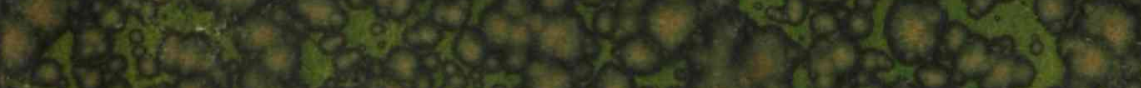

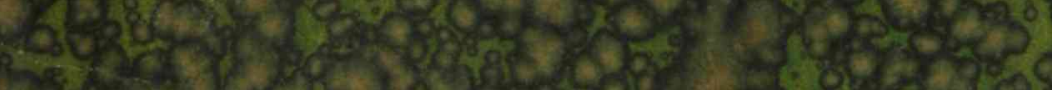

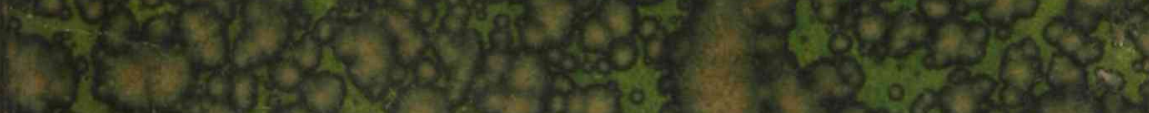

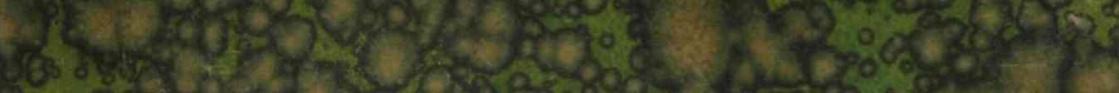

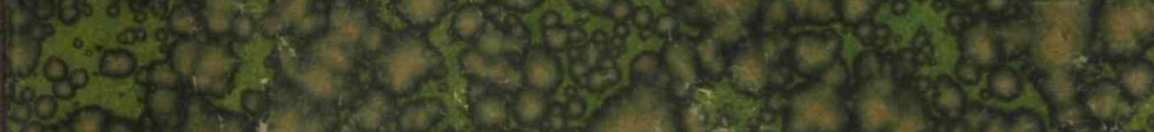

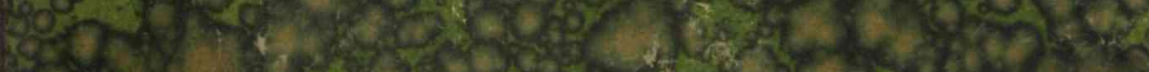

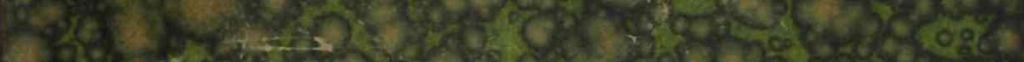

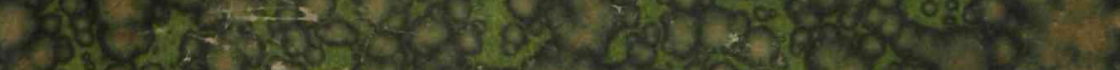

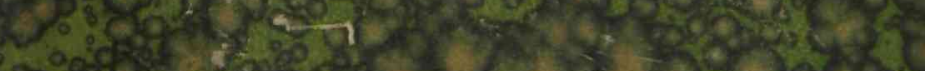

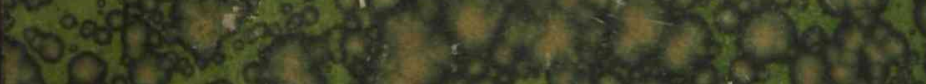

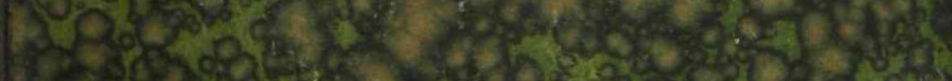
2.

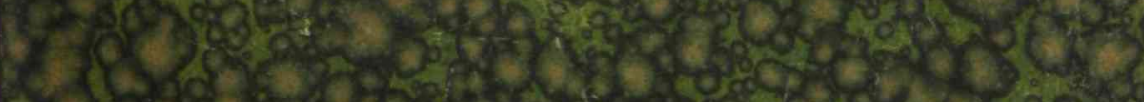

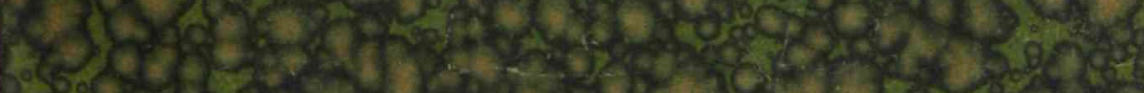

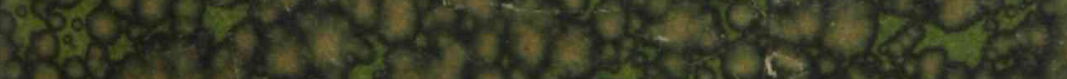

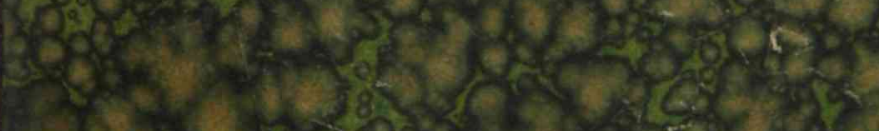

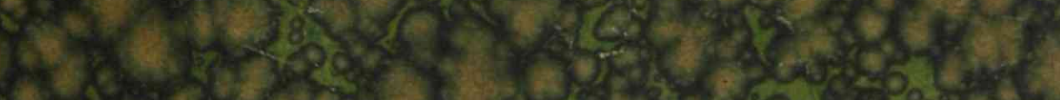

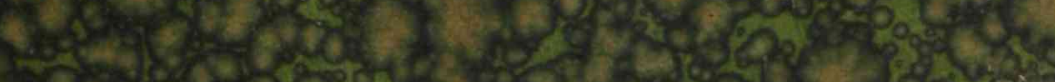

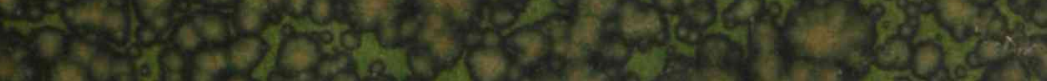
Cida

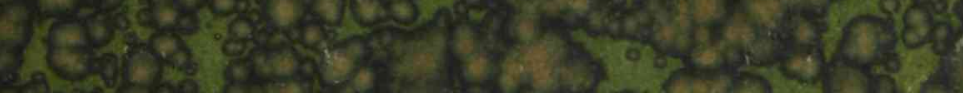





\section{LA SOCIOLOGIE}

SA NATURE, SON CONTENU, SES ATTACHES 


\section{OUVRAGES DE M. RENE WORMS}

De la volonté unilatérale considérée comme source d'obligations. - Paris, Giard et Brière, un vol. in-80 de 204 pages, 1891. Epuisd.

Précis do philosophie, d'aprés les Legons de philosophic de M. E. Rabier. Paris, Hachette, un vol. in-16 de 4 io pages, 1891 . Quatrième édition, 1911. Epaisé.

Flóments de philosophie scientifique et de philosophio morale. - Paris, Hachette, un vol. in-16 de 114 pages, 1891.

La moralo de 8 pinoza. - Mémoire couronné par l'Institut (Academie des Sciences morales el politiques). - Paris, Hachetle, un vol. in-16 de 384 pages, 1892. Epaise. Traduit en russe.

- De natura et methodo sociologiø ..- Paris. Giard et Briére, un vol. in-80 de rob pages, 1896 . Epuise.

Organisme et société. - Paris, Giard el Brière, un vol. in-80 de 410 pages, 1896 . Epuisé. Traduit en russe

La science ot l'art en économie politique. - Paris, Giard el Brière, un vol. in-18 de 131 pages, 1896 .

Philosophie des sciences sociales. - Paris, Giard el Briere, 3 rol. in-8e :

Tome I : Objel des sciences sociales; 230 pages, 1903. Seconde èdition, 1913,

Tome II : Méthode des sciences sociales : 254 pages, 1904. Seconde édition, 1018.

Tome III : Conclusions des sciences sociales: 310 pages, 1907. Seconde edition, 1421.

trudes d'óconomie et de légielation rurales. - Paris, Giard et Briire, un vol. in.18 de 304 pages, 1906.

Les principes biologiques de l'érolution sociale. - Paris, Giard et Brière, un rol. in.18 de 122 pages, 1910. Traduit en russe.

La sexualite dans los naissances françaisos. - luvrage couronné par l'Institul (Académie des Siciences). - Poris, Giard el Brière, un vol. in-80 de 240 pages, 1 !1 2.

Les associations agricoles. - Paris, Giard el Brierc, un vol. in-18 de 230 pages, 1914 .

Natalité ot régime successoral. - Mémoire recompensé por la Faculte de droil de l'lenirervile de Paris fcoecours Kussil. - Paris, Payol, un vol. in-16i de 2soplaters. 1917.

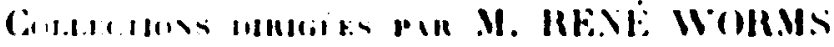

Revue internationale de soclologie - Marn, liarid it Brière. at volumes krand int-80

Annales de l'Institut international de soclologio. - Paria, Giard et liriere, ih volumes in-to.

Btbllothdque sociologique internationale. - Paris, tiiard el IBriere, sb volumes ill-80 et 10 rolume, in-18. 
BIBLIOTHĖQUE SOCIOLOGIQUE INTERNATIONALE publlée sous la direction de M. René WORMS

Secrétaire général de l'Institut international de sociologie

$$
\text { SÉRIE in-I } 8-J .
$$

\section{LA

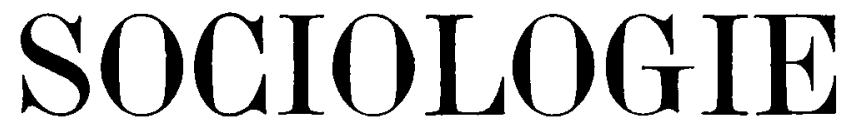

\section{SA NATURE, SON CONTENU, SES ATTACHES}

PAR

\section{RENÉ WORMS}

DOCTEUR EN DROIT, ÈS LËTTRES ET ĖS SCIENCES, AGRÉGÉ DE PHILOSOPHIE, AGRÉGÉ DES SCIENCES ÉCONOMIQUES, DIRECTEUR DE LA REVUE INTERNATIONALE DE SOCIOLOGIE

\section{PARIS $\left(5^{\mathrm{e}}\right)$}

Anciennement M. Giard \& E. Brière Marcel GIARD \& $\mathrm{C}^{\mathrm{ie}}$, successeurs LIBRAIRES-ÉDITEURS 16, rue Soufflot, et 12 , rue Toullier 



\section{A LA MÉMOIRE DE MON PĖRE}

\section{ÉMILE WORMS}

PROFESSEUR hoNoraIRe D'Économie politique

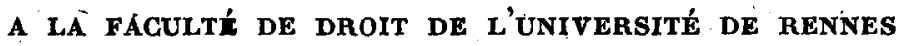
CORRESPONDANT DE L'INSTITUT DE FRANCE 



\title{
PREMIÈRE PARTIE
}

\author{
NATURE DE LA SOCIOLOGIE
}

\section{CHAPITRE PREMIER}

POSITION DE LA QUESTION.

Les sciences ont, en général, un objet nettement défini. Sans doute quelque hésitation peut régner, dans certains cas particuliers, sur leuis frontières respectives. Mais du moins le point sur lequel d'ordinaire portent les efforts de chacune d'elles est fixé avec précision. Le nom de chacune équivaut à une définition, acceptée de tous.

Il n'en est pas ainsi de la sociologie. Pour celle-ci, c'est son concept qui est en cause. Sous le nom de sociologie, en effet, on entend les choses les plus diverses. Certains esprits hésitent même 
à croire que ce nom puisse désigner un ensemble de recherches véritablement scientifiques.

D'où viennent ces incertitudes et ces doutes ? La faute en est-elle à la nouveauté du vocable adopté? Inventé par Auguste Comte, introduit par lui en I 842 dans le tome IV de son Cours de philosophie positive, pour désigner les travaux que jusque-là il rangeait sous le terme de physique sociale (I), le mot a déjà un assez long passé pour que son sens ait pu devenir précis. En estelle à la singularité de ce vocable? Il y a un tiers de siècle, on lui reprochait d'être mal formé, hybride, composé d'une racine latine et d'une désinence grecque ; on l'écrivait volontiers entre guillemets, comme les mots peu usités ou étrangers. Aujourd'hui on a renoncé à cette attitude dédaigneuse. Le terme de sociologie s'est fait recevoir dans le langage courant. Les adversaires de l'idée acceptent du moins l'expression.

Les vraies causes des hésitations dont nous parlons se trouvent ailleurs, et elles sont multiples. D'abord, les sociologues doivent s'en imputer une à eux-mêmes. Il n'ont pas assez songé à se mettre d'accord entre eux. Trop souvent, chacun d'eux a visé avant tout à affirmer son originalité, en délaissant les voies tracées par ses prédécesseurs, en s'éloignant de celles que prenaient ses

(I) Terme qu'il a abandonné lorsque le savant belge Adolphe Quetelet l'eut employé pour désigner des travaux d'anthropologie et de démographie. 
contemporains. Beaucoup ont voulu apporter une conception nouvelle de la sociologie; en proposer un definition originale. Il est tout naturel que les non-initiés, nous voulons dire les hommes d'étude non spécialistes en la matière, n'aient vu que confúsion dans cette multiplicité de systemes divers. Les inventeurs de ces derniers en sont, en grande partie, responsables:

Un autine facteur a agi dans le mềme sens, et ce dernier était du fait, non plus de la sociologie, mais des disciplines voisines. Quand le terme de sociologie a été inventé, le domaine social était déjà exploré par de nombreux chercheurs. Il exigtait une économie politique, une morale, un droit, une politique. Ou plutot, il existait de multiples écoles économiques, morales, politiques. On comprend que leurs membres aient considéré là nouvelle venue comme une intruse, d'autant qu'elle les traitait trop souvent, de son côté, avec un dédain peu déguisé et peu.justifié. Les polémiques qui se sont engagées entre eux et les sociologues ont envenimé le débat théorique sans l'éclaircir. $\mathrm{Au}$ lieu de chercher pour la sociologie un terrain distinct, où elle pût se développer sans heurter les études antérieures, on a préféré lui contester tout droit à l'existence. On l'a déclarée săns objet possible, ce qui était à coup sûr exágérer.

La conciliation n'eat pourtant point été, ce nous semble, fort malaisée. L'économie politique, la morale, le droit, la politique, surtout tels qu'ils étaient au temps d'Auguste Comte, aspiraient d diriger le monde social plus qu'à le connaftre. 
Ils étaient (suivant une distinction que nous éluciderons bientôt) moins des sciences que des arts. S'ils avaient bien voulu en convenir, un malentendu fondamental aurait pu être évité, qui subsiste encore aujourd'hui. Ce n'est que plus tard, et surtout de nos jours, que se sont constituées, en face de ces arts sociaux, des sciences sociales proprement dites, qui toutes sont des fragments de l'histoire sociale :' à savoir l'histoire économique, l'histoire des mœurs (que 1'on intitule déjà, en anticipant quelque peu, la science des mours), l'histoire du droit, l'histoire des institutions politiques. Celles-ci ont avec la sociologie une relation plus directe, elles en sont plus proches, et de ce contact même peuvent naître de nouvelles rivalités fondées sur un désir mutuel d'absorption. Mais du moins elles ş'y sentent apparentées, et la réciproque est également vraie, de sorte que le débat ici a quelque chose de plus familial. Ce débat aussi, du reste, a contribué à faire naître certains doutes sur la tâche propre de la sociologie. Pourtant, s'il est bien conduit, aucun ne jettera sur elle une lumière plus vive et plus pénétrante.

En somme donc, par la faute des sociologues, par celle des représentants des anciens arts sociaux, par celle des tenants des jeunes sciences sociales, l'hésitation règne en ce qui concerne l'essence de la sociologie. Ce n'est pas seulement le détail de son contenu qui est contesté, ce sont ses principes fondamentaux eux-mêmes, c'est sa définition et jusqu'à la possibilité de son existence. 
Il y a là uné situation dònt, à coup sû́r, il importe grandement à cette discipline de sortir sans aucun retard.

Cela n'importe pas qu'à elle seule. De divers côtés, beaucoup d'espérances ont été fondées sur la sociologie ; pour qu'elles puissent, fût-ce partiellement, se réaliser, il faut d'abord que les questions dont nous venons de parler aient été résolues. On a cru parfois que la sociologie serait amenée à rénover les sociétés humaines. On a estimé, d'un autre côté, que l'introduction du point de vue sociologique serait de nature à transformer toutes les scienoes et la philosophie. Qu'il $y$ ait là beaucoup d'exagération, nous l'admettons très volontiers, et nous comptons nous-même le montrer. Il reste toutefois exact, à nos yeux, que la sociologie est susceptible d'apporter son contingent à l'action sociale et à l'ensemble du savoir, qu'elle contient des idées fécondes à de multiples égards: Si on veut qu'elle joue son rôle utile, il faut d'abord que son concept propre soit nettement dégagé, qu'on sache bien oe qu'elle est, ou du moins ce qu'elle pourra être. C'est à fixer les idées sur ce point que nous allons nous attacher. 


\section{CHAPITRE II}

\section{LA SOCIOLOGIE N'EST PAS UN ART.}

Afin de déterminer l'objet de la socialogie, il importe d'écarter immédiatement une idée fausșe qu'on s'en fait trop souvent,

Le public, même le publịc lettré, croit en génér ral que la șociologie a pour but la réforme des institutions sociales, qu elle vise directement à une réorganisation de la société, Pour lui, le sociologue est celui qui fait " du bon socialisme ". En ce cas, sociologie serait à peu près synonyme de "l économie sociąle ".

Peut-être ne devons-nous pas trop regretter que le langage courant ait commis cette confusion. Car elle a contribué à faire accepter, à faire même aimer le nom de la sociologie. Si ce terme est devenu assez populaire actuellement, c'est justement parce qu'on a cru voir dans la sociologie une bonne fée s'apprêtant à guérir les maux de notre pauvre humanité.'A coup sûr, son succès aurait été bien moindre si la sociologie n'était 
apparue que comme une étude d'ardre scientifir que ou phílosophique,

Et pourtant, telle est incontestablemẹt sa nature, du moins d'après tous ses adeptes les plus aưtorisés. Le penseur illustre qui l'a fondée, Aưguste Comte, insište, dès le début de la seconde leçon de gan Cours de philosophie p.ositive, sur la distinction qu'il y a lieu de faire entre les sciences et leurs applications, et il classe la sociolọgie au sommẹt de la hiórarchie des sciences fondamentales. Les longs développements qu'il lui consacre ensuite, et qui, constituent les tomes IV, $V$ et VI de ce Cours, sont tous de nature philosophique ou historique. Pour eux, il ajourne momentanément tout projet de réorganisation sociale (I).

Tel est aussi le cas de l'homme éminent qui après Comte a le plus contribué à la création de la sociologia, Herbert Spencer. Pour lui, la sociologie est une f́tude de la façon dont se sont spontanément organisées les sociétés humaines et dont elles évoluent. Recueillir des faits à cet égard,

(I) Il est vrai que plus tard Comte, arrivant à cette tentative de réorganisation, écrira le Système de politique positive ou Traité de sociologie instituant la religion de l'humanité. Il semblera ainsi confondre la sociologie avec la politique et la religion. Mais justement catte attitude de Comte a été blâmée par ses disciples les plus qualifiés, tels que Emile Littré et John Stuart Mill, qui l'ant abandonné lorsqu'il a ainsi renoncé à la méthode objective pour adopter la méthode subjective. 
les classer, dégager les lois générailes de leur processus naturel, est sa seule préoccupation dans sa Sociologie descriptive et dans ses Principes de sociologie. Les applications pratiques n'y apparaissent pas. .

Il en est de même chez les sociologues ultérieurs. En France, Alfred Fouillée, Gabriel Tarde, M. Alfred Espinas, Emile Durkheim voient dans la sociologie une recherche soit philosophique, soit scientifique, mais non pas pratique. C'est également ce que firent, en Belgique, M. Guillaume De Greef ; en Russie, Eugène de Roberty ; en Autriche, Ludwig Gumplowicz. En Allemagne, où la sociologie n'a pénétré que plus récemment, elle a dès le début affirmé ce caractère dans les écrits des professeurs Ferdinand Toennies et Georg Simmel. 'Un fait significatif à cet égard est le suivant. En IgIo, à Francfortsur-le-Mein, la Société allemande de sociologie tenait son premier congrès national $:$ le règlement ne permettait aux orateurs que de constater des faits, non de les apprécier, et les comptesrendus du congrès nous montrent les présidents de ses séances successives faisant une véritable chasse aux jugements de valeur ("Werturteile ") auxquels les auteurs des communications voulaient parfois, à la dérobée, se livrer (I).

(1) Voir Robert Michels, La Société allemande de sociologie et son premier congrès, article publié dans la Revue internationale de sociologie, I910. Cf. Verhandlungen des ersten deutschen Soziologentages, Tübingen, Igrr.' 
Aux Etats-Unis, il est vrai, le terme de sociologie a longtemṕs revêtu côte à côte l'acception scientifique et l'acception pratique, et elles sont restées quelque peu intriquées l'une dans l'autre' (I). Toutefois, les principaux sociologues tendent à dégager la première. Lester $\mathrm{F}$. Ward a écrit sa Pure Sociology bien avant de composer son Applied Sociology et il attachait surtout de l'importance à celle-là. Les Principles of Sociology du professeur Fr. H. Giddings sont essentiellement une ouvre de science.' Dans les programmes récents des Universités américaines, la sociologie 's'est détachée de ses liens avec ce qu'on appelle là-bas "Charities and correction ". Partout en un mot, dans la langue des techniciens, le mot de sociologie se dépouille de sa signification d'ordre pratique.

Essayons de réduire à une formule ce qui résulte de là. Une distinction, qui se fait de jour en jour accepter davantage, va nous en fournir le moyen. C'est la distinction entre la science et l'art. Il faut bien se garder de la confondre avec la distinction, plus anciennement connue et non. moins vraie, entre la théorie et la pratique. La théorie, c'est ce qu'on devrait réaliser ; la pratique, c'est ce qu'on peut réaliser. La théorie est d'ordre général et abstrait ; la pratique est

(1) Voir Albion W. Small, Fifty years of sociology in the United States, travail paru dans The American Journal of Sociology, may rgi6. 
d'ordre particulier et concret. Mais, ai elles diffèrent ainsi en un point très important, elles se ressemblent sur un point plus capital encore peutêtre. C'est que toutess les deux visent à l'action. L'une dans des sphères éleyées, l'autre dans des sphères modestes, ont le même but: influer sur le monde existant, pour l'organiser selon nos intérêts ou selon noọ désirs, - La science fait autre chose. Elle n'aspire pas à agir, mais seulement à connaître, Elle ne vout pas donner au monde une orientation nouvelle, elle cherche seulement comment il s'oriente spontanément. Elle ne songe pas à transformer, mais bien à informer. - On voit par là quelle est sa différence d'avec l'art. Celuici tend à l'idéal; la science tend au réel. Il formule des préceptes, elle dégage des lois. Il veut influer sur l'avenir; elle porte ses investigations sur le passé et sur le présent. Il a forcément un caractère subjectif; elle vise à être purement objective. - L'art, en somme, c'est ce qu'on appelait la théorie quand on distinguait celle-ci de la pratigue. Mais la science ne fait pas de thérorie, elle fait des constatations, ou du moins le mot de théorie ne désigne chez elle qu'un ensemble de notions positives, - En matière sociale, l'humanité s'est longtemps bornée à des pratiques. La théorie, ou l'art, n'a paru que plus tard. La science est la dernière venue. L'óvolution s'est faite plus vite en ce qui concerne les relations de l'homme avec le monde physique et le monde arganique; ici, la matière étant mains complexe, la science a pu se constituêr plus tôt. Au contraire, 
pour la vie sociale, il existait sans doute, depuis longtemps, sous le nom d'histoire, des recueils 'de faits, mais ce n'est qu'au dix-neuvième siècle qu'on a compris l'intérêt que présente l'organisation proprement scientifique de ces matériaux. Cet intérêt est grand, à deux points de vue au moins. D'abord, il faut une science pour guider l'art. On ne peut, en effet, agir profondément et largement sur le monde social qu'en sachant parfaitement ce qu'il est. Puis, la science a par elle-même, et en dehors de cette application, une haute valeur. Elle satisfait les plus nobles asplrations de l'esprit, elle est éducative au premier chef, elle donne à l'homme la pleine conscience de son lien avec ce qui l'entoure, de la dépendance réciproque de tous les êtres et de leur solidarité. C'est ce qui fait que, de nos jours, elle s'est si vịte et si amplement développée.

La sociologie est précisément née de ce grand mouvement qui a porté nos contemporains vers la constitution de la science. Elle n'est pas une pratique, elle n'est pas un art, elle est une recherche d'ordre scientifique. De quel ordre au juste? c'est ce que nous allons nous demander.

(I) Pour l'analyse des idées ci-dessus exposées, nous nous permettrons de renvoyer à des travaux personnels antérieurs : tels notre mémoire sur La science et l'art en matière sociale, insóré dans le tome Ior des Annales de l'Institut international de sociologie; notre livre intitulé La science et l'art en économie politique ; et deux ehapitres de notre ouvrage plus étendu sur la Philosophie des sciences sociales (tome I, chapitre IX, et tome III, chapitre $\mathbf{X I}$ ). 


\section{CHAPITRE III}

LA SOCIOlogié N'EST PAS UNE SCIENCE SPÉCIALE:

Le mouvement dont nous venons de parler a amené, au $\mathrm{XIX}^{\mathrm{e}}$ siècle, la constitution d'une série de sciences sociales particulières. Chacune d'elles s'efforce de suivre le déroulement d'un ordre de faits sociaux à travers le temps et l'espace. Ce sont, entre autres, l'histoire économique, ou science économique, distincte de l'art économique (I); l'histoire des mours, ou science des mœurs, distincte de la morale (2); 1'histoire du droit, ou science juridique, distincte de l'art du législateur et de l'art du, juge ou de l'avocat; l'hiștoire des institutions politiques, ou science

(1) Voir Fernand Faure, article Science et art, dans le Dictionnaire d'économie politique publié sous la direction de Léon Say.

(2) Voir L. Lévy-Bruhl, La morale et la science des mours. 
politique, distincte de 1'art politique. Quelle place reste-t-il, à côté de ces sciences, pour la sociologie?

L'idée la plus simplè à ce sujet, c'est que la sociologie pourrait porter sur un domaine que ces diverses sciences délaissent. Chacune de celles-ci s'attache à un ớdre de phénomènes sociaux. Mais, avant de considérer les phénomènes, ne faut-il pas connaître les éléments qui les manifestent, et le groupement de ceux-ci ? C'est ainsi que, dans le domaine biologique, avant d'examiner les fonctions, on décrit les organes. Dans le domaine social, il y a, de même, place pour une ou plusieurs sciences des organes. Celles-ci décriraient les structures sociales, c'est-à-dire les divers assemblages formés par les éléments de la société. Ne pourrait-ce être là l'objet de la sociologie?

Cette conception a revêtu elle-même deux aspects, l'un plus populaire, 1'autre plus scientifique. Chacun d'eux mérite d'être caractérisé brièvement.

Dans le langage courant, on entend sans cesse parler de " la question sociale ", pour désigner la question de la lutte des classes. Il semble que le problème social par excellence soit celui de l'opposition existant entre patrons et ouvriers. Ces deux masses, devenues cohérentes, menacent fréquemment de s'entre-choquer dans un grand combat, sans compter leurs quotidiennes escarmouches. Karl Marx, qui a appelé l'attention des savants sur ce fait, qui l'a précisé et qui en a 
fait la thécrie générale; passe aux yeux de nombre de gens pour avoir par là rénové ou même inventé la sociologie.

Il est à peino besoin de dire ce que cetto vue a d'erroné. Sans doute la lutte des classes est un fait considárable, qu'il y a d'ailleurs lieu de déplorer et qu'il faudrait s'efforcer d'atténuer et même de supprimer. Mais il en est de plus importants encore. La lutte des nationalités est de ce nombré, et à gertaines heures celle-là est momentanóment oubliée pour celle-ci. C'est dire que le débat et la conciliation des classes ne peuvent occuper souls tout l'art social. Pareillement, la descriptian des classses est une question importante pour les sciences sociales proprement dites, mais elle n'esst pás leur objet unique. Mềme, elle ne forme qu'une partie de la science des groupements sociaux. Car, parmi ces groupements, il y a à ognsidérer - en dehors des classes - les races, les familles, les tribụs, les cités, les Etats, les professions, les aśsociations volontaires, tous modes d'organisation sur lesquels nous comptons pevenir. On no voit pas dès lors pourquai la sociologie négligerait ces derniers au profit des clas oss soules. L'examen do celless-ci est à coup sûr instructif et important; il ne saurait toutefois, à lui soul, constituer la sociologie tout entière.

Mais n'y a-t-il pas, dans cette idée fausse, le germe d'une idée uraie? Si la description des classes n'est pas la sociologie, celle-ci ne seraitelle pas la description do tous les groupements sociaux? Quelques hommes d'étude l'ont pensé 
et, if y a une vingtaine d'année, certains l'ont écrit, C'est là la forme scientifique de la conception dont nous parlons. Laissant à la science économique, à la science des mours, à la science des religions, à la science juridique et à la science politique l'examen des fonctions sociales, la socior logio prendrait pour elle l'examen des formes sociales. Elle étudierait les éléments humains de la société et leurs relations avec les éléments nonhumains: sol, milieu cosmique, espèces minérales, végétalęs et animales. E1le étudierait aussi leurs divers genres d'agrégation, dont les principaux viennent d'être indiqués. Et cette double desscription pourrait servir de base, de substrat, à celle que les sciences de fonctions feraient de la viè sociale dans sa riche complexité.

Ceette façon de voir, ou plutôt de s'exprimer, n'a point prévalu. On s'est aperçu que certaines recherches sur les éléments sociaux et leurs groupements avaient déjà été instituées par des sciences auxquelles on ne pouvait prétendre les enlever. Deux de ces sciences, relativement assez anciennes, étaient l'ethnographie, étude des races, et la démographie, étude de la population et de ses subdivisions. Une autre, beaucoup plus jeune, mais fort active, s'intitule la géographie humaine ou géographie sociale et porte son enquête sur les rapports de l'homme et de son milieu" "tellurique ". Quant à l'ensemble de ces recherches, on a compris qu'il ne pouvait être toute la sociologie. Les sciences de fonctions étaient, en effet, aussi fondées que lui à revendi- 
quer ce nom. On a alors inventé pour cet ensemble un vocable nouveau, celui de " morphologie sociale " (I). Par là, on a reconnu qu'il constitue simplement une science sociale particulière, et on s'est conformé au désir qu'avaient d'instinct tous les sociologues qualifiés, de donner la plus large acception possible au terme de sociologie. Et l'on a eu raison. De même que la cosmologie et la biologie rayonnent sur toute la nature physique et sur toute la nature vivante, de même la sociologie doit rayonner sur toute la nature sociale. C'est ainsi qu'Auguste Comte, son créateur, l'avait conçue et dans une certaine mesure constituée. I1 n'y a pas de motif suffisant pour renoncer à sa définition. Le terme par lui trouvé ne doit pas être monopolisé au profit d'une seule branche d'études, si utile qu'elle puisse être. La sociologie ne saurait se ranger parmi les savoirs spéciaux (2).

(I) C'est celui sous lequel il est désigné dans L'Année sociologique.

(2) Nous avons essayé de dresser une liste des sciences sociales particulières, suivant un ordre logique, dans notre Philosophie des sciences sociales (tome Ier, Objet des sciences sociales, chap. $\mathrm{X}$ et $\mathrm{XI}$ ). 


\section{CHAPITRE IV}

EN QUEL SENS LA SOCIOLOGIE EST LA SCIENCE GÉNÉRALE DES SOCIÉTÉS.

Nous venons d'indiquer que la sociologie n'est, à notre sens, ni un art social, ni une science sociale particulière. Il en résulte qu'elle ne peut être qu'une seule chose: la science générale des sociétés: Nous demeurons fidèle sur ce point à la pensée d'Auguste Comte. Mais la science qu'il avait créée s'est fort développée depuis lors. De sa croissance sont nés de nouveaux problèmes. Il ne nous suffit plus, comme à lui, de poser une définition d'ensemble. Il importe de donner des précisions.

En effet, la définition qui précède peut être prise en deux sens différents. En disant que la sociologie est la science générale des sociétés, on peut entendre: ou bien, qu'elle en est la science intégrale, ou bien, qu'elle est la science de leurs aspects généraux. L'une et l'autre de ces conceptions a sa raison d'être et ses partisans. 
E. Durkheim admet la première d'entre elles. Pour lui, "la sociologie n'est et ne peut être que le système, le corpus des sciences sociales (I)". Celles-ci sont, à vrai dire, les membres de la sociologie. Dans les volumes successifs de L'Année sociologique, on les voit traitées comme telles. Ainsi, cette publication résume les travaux récents portant sur ce qu'elle appelle la sociologie religieuse, la sociologie morale, la sociologie juridique, la sociologie économique. La science des religions, la science des mours, la science du droit, la science éçonomique sont considérées comme des fragments de la sociologie, fragments qui doivent, naturellement, s'inspirer de communs principes. La sociologie consiste dans leur juxtaposition, dans leur intégration; elle est la somme de coș élóments additionnés.

Voici maintenant la seconde conception. Suivant elle, les sciences que nous venons de nammer ont chacune son indépendance et ses caractêres distincts. La science des religions est trop différente de la science du droit, la science des moeurs l'est trop de la science économique, pour qu'on puisse leur appliquer un même nom. Elles doivent donc être d'ordinaire cultivées par des cherrcheurs différentá : les nécessités de la division du travail scientifique l'exigent. Seulement, il

(z) E. Durkheim et P. Fauconnet, Saciologie ét sciences sociales, artiele inséré dans la Rezue philosophique, mai 1993, page 465 . 
y a place pour des rapprochements entre ces sciences. Chacune étudie un des aspects de la réalité sociale. Quand elles ont, séparément, pousgé leurs tâches propres aussi loin que possible, il y a liẹu de comparef leurs résultats, de less associer, pour se faire une id́s d'ensemble du tout dont chacune d'elles a examiné un fragment. C'egt justement le rôle de la sociologie. Celle-ci n'absofbe donc pas en elle les sciences sociales particulièzes. Elle se présente plutôt comme leur couronnement général. Elle n'est point tout leur édifice, elle en forme seulement le faîte commun.

Des deux conceptions qui viennent d'être indiquées, laquelle est préférable? Avant de choisir entre elles, il est équitable de limiter le débat. En fait, la divergence est assez mince. Durkheim ne nie pas qu'il puisse y avoir place, à côté des " sociologies particulières ", pour une étude des résultats généraux. Voici ses propres expressions à cet égard. "Certes, si l'on entendait dire simplement que, une fois les sciences sociales suffisammant avancées, il y aurait lieu de rapprocher les uns des autres les résultats obtenus par chacune d'elles, afin d'en dégager les rapports les plus généraux qui y sont inclus, le problème ainsi posé n'aurait rien en soi d'insoluble (I) ". Et réciproquement, dans la seconde conception, on admet naturellement qu'il y a intérêt, pour tout adepte de chacune des sciences sociales parti-

(I) Ibidem, page 477, 
culières, à recevoir une large instruction portant sur les sciences sociales voisines, à se tenir au courant des progrès de celles-ci, à s'inspirer de leurs données dans ses recherches personnelles, à sentir la solidarité qui 1'unit à ses confrères voués à ces sciences, à agir en un mot comme si toutes celles-ci et la sienne propre formaient une unité. - Cela bien compris, la distinction des deux conceptions se réduit à peu près à une différence de terminologie. Pour Durkheim, la sociologie est le total des sciences sociales, ét, si l'on veut donner un nom à l'étude 'de leurs résultats d'ensemblè, il faut l'appeler sociologie générale ( $\mathrm{I}$ ). Dans l'autre conception, les sciences sociales sont en principe indépendantes, et l'examen de leurs résultats d'ensemble s'appelle sociologie, sans qu'il soit besoin d'accoler à celle-ci l'épithète de générale, que sa nature implique nécessairement (2).

Le débat étant ainsi réduit à une question de mots, on pourrait peut-être s'abstenir de le trancher. Les définitions de mots ne sont-elles pas libres? Toutefois, 1'importance de la terminologie est telle, que nous croyons devoir indiquer

(I) Une rubrique portant ce dernier titre figure dans L'Année sociologique.

(2) Voici donc un tableau de concordance des expressions employées des deux côtés, celles de Durkheim étant à gauche :

sociologies spéciales $=$ sciences sociales particulières ; sociologie généràle $=$ sociologie 
nos préférences. Nous les tirerons de ce qui paraît être l'intérêt de la sociologie. L'un des grands obstacles que rencontre actuellement sa diffusion vient de la résistance que lui opposent de nombreux spécialistes. Ils 'craignent en effet de voir absorber par elle leurs études propres, ils redoutent ses tendances envahissantes. Cette crainte est en réalité peu fóndée quand il s'agit des tenants des arts sociaux, puisque la sociologie s'interdit, au moins directement, les applications. Elle l'est beaucoup plus chez les adeptes des sciences sociales particulières. Un économiste éminent, Emile Levasseur, qui était accueillant aux idées nouvelles sans sé croire tenu de les accepter toutes, -s'est fait l'écho de ces sentiments dans une allocution remarquable qu'il adressait aux sociologues. Elu par ceux-ci président pour I906 de l'Institut international de sociologie, il prononçait au $6^{\mathbf{e}}$ congrès de cette association, à Londres, un discours d'ouverture où il les mettait en garde contre d'excessives prétentions. "Conseillons, disait-il en terminant, la modestie aux sociologues (I) ". C'est de ce sentiment de modestie que nous croyons devoir nous inspirer dans le cas présent. La , sociologie se fera mieux accepter, si elle limite ses aspirations. Qu'elle renonce à être l'intégralité des sciences sociales, et on admettra sans doute qu'elle peut en être le

(I) Annales de l'Institut international de sociologie, tome XI, 1907, page 64 . 
rapprochement: Si elle ne menace pas l'indépendance des sciences sociales particulières, celles. ci en retour ne nieront pás son droit à l'existence. Par des concessions réciproques, les domainess se délimiteront au mieux des intérêts de tous, et de céux de la science elle-mêmé. Il y a quelque mérite, mais il y a aussi quelque avantage, a savoir se borner. La sociologie doit le falre, si elle veut vivie en paix avec ses vóisines et trouver en elles des alliées. Engãgeons-la donc à ne pas débof́der leurs frontières et à se contenter, s'il est pérmis d'ainsi dire, de les "survoler ". Coñcluons en disant que nous préférons la définir, non pas l'intégralité des sciences sociáles, mais leur synthèse. 


\section{CHAPITRE 'V.}

\section{LA SÓCIOLOGIE EST LA PHILOSOPHIE DES SCIENCES SOCIALES PARTICULIỂंES. ।}

Du caractère que nous venos de reconnaître a la sociologie, résulte une conséquence qui doit être mise en lumière, et qui servifa de conclusion aux précédentés recherches. La sociologie, dlsions-nous, domine les sciences sociales particulières. En d'áutres termes, celles-ci se divisent le monde social, et celle-là en reconstitue l'unité. Celles-ci donc ont chacune un objet propre, celle-là envisage lęurs objets à toutes à un point de vue supérieur. A dire vrai, celles-ci seules sont proprement des sciences ; celle-là est plutôt la philosophie de ces mêrnes études. Et c'est ainsí que nous arrivons, au terme de notre enquête, à définir la sociologie comme constituant la philosophie des sciences sociales particulières.

Il est inutile de donner lci l'énumération des caracteres qui distinguent la philosophie d'avec la science proprement dite. Ils ont été mainte 
fois relevés. Même quand il s'agit d'une philosophie positive ou philosophie des sciences, chacun sait qu'il y a là un ordre de travaux assez. différents des travaux de pure science. Auguste Comte notamment, y a insisté, dans la seconde leçon (déjà citée par nous) du Cours de philosophie positive. Ces deux ordres se distinguent comme l'analyse de la synthèse. La science constate des faits particuliers et les résume en des lois.' Mais les problèmes dominants sont du ressort de la philosophie. La philosophie d'une science est l'examen des questions les plus générales qui se posent au sujet de celle-ci. Les unes sont des questions initiales; les autres, des questions finales. Au début de la science, il s'agit d'en fixer l'objet et la méthode. Au terme de la science, il y a lieu d'en résumer les conclusions. Si l'on applique cette notion dans notre cas, on voit que la sociologie a deux fois l'occasion de procéder à sa tâche d'unification. A la base, elle trace à chacune des sciences sociales particulières son domaine et lui indique les procédés dont elle peut se servir. Naturellement, elle rapproche ainsi ces sciences les unes des autres. Car, en fixant les domaines, elle essaie d'éviter, d'une part toute lacune, d'autre part tout double emploi et tout empiètement. Et, en fixant les procédés, elle tấche de faire bénéficier chaque science sociale particulière de l'expérience de toutes, leur indiquant comment une méthode qui a réussi à 1'une pourrait être adaptée à l'usage des autres. Au sommet, elle réunit les résultats de ces disciplines 
particulières; elle montre comment les diverses faces de la réalité sociale, provisoirement dissociées pour les besoins de l'étude, se rejoignent et se relient dans la vie effective, comment tous les éléments sociaux collaborent, toutes les fonctions sociales' s'enchevêtrent, tous les stades de l'évolution se continuent et s'enchaînent. Elle opère donc bien, à ces deux moments successifs, l'œuvre synthétique que nous avions reconnue comme la sienne.

Mais y a-t-il vraiment lieu de distinguer ces deux moments? Théoriquement, oui sans doute. Effectivement, beaucoup moins. C'est en se plaçant à un point de vue idéal qu'on peut les différencier. Si on suppose le travail des sciences sociales achevé, il est logique de mettre à chacune d'elles une introduction et une conclusion, et de voir dans cette introduction et dans cette conclusion autant de chapitres de la sociologie générale. Seulement, il est à peine besoin de dire que ce monument est fort loin d'être bâti. Tant qu'il ne le sera point, il faudra travailler à la fois aux différentes parties de l'édifice. Le corps de la science, son introduction et sa conclusion s'élaboreront ainsi pari pass̀u. La sociologie se construira donc en même temps que les sciences sociales' particulières et dans la même mesure qu'elles. Et ses deux opérations, l'initiale et la finale, se réaliseront simultanément, si singulier que cela puisse paraître. Nous serons, par conséquent, amené à ne pas les trop séparer dans ce worus 
qui va suivre. Tout en les distinguant, nous ne les opposerons pas.

Ce que nous allons tenter de faire voir, c'est que les recherches précédentes ont une réelle portée. Nous avons déterminé, dans l'abstrait, le rôle qui incombe à la sociologie. Nous voudrions maintenant montrer comment, dans le concret, elle l'a rempli, ou du moins a commencé à le remplir. Il s'agit donc de marquer ce que la sociologie a fait jusqu'à présent, quels résultats elle a déjà enregistrés. Bien entendu, cela ne saurait s'opérer qu'à grands traits, puisqu'on n'attend pas de nous, ici, un traité complet de sociologie. Mais du moins faut-il dresser, en quelque sorte, le sommaire d'un semblable traité, et faire connaître quelles en seraient les lignes mâtrtresses. Après avoir indiqué l'objet logique de la sociologie, nous allons esayer de dire ce qu'en est le contenu effectif. 


\title{
DEÚXIÈME PARTIE
}

\section{CONTENU DE LA SOCIOLOGIE}

\author{
CHAPITRE VI \\ LA SOCIÉTÉ ET LES RELATIONS SOCIALES.
}

L'ensemble des êtres qui constituent l'univers sensible nous paraît pouvoir être divisé en trois grandes catégories: les corps bruts, les êtres vivants, les sociétés. Il forme donc trois domaines, que l'on peut appeler respectivement l'inorganique, l'organique, le supra-organique. Ces domaines, qui sont à certains égards juxtaposés, sont aussi, au point de vue le plus important, superposés. Car le supra-organique est fait d'éléments' organiques, comme l'organique est fait 
d'éléments inorganiques. Chacun d'eux est caractérisé par un mode d'agencement inconnu au précédent. Il prend à celui-ci sa matière et lui donne une forme nouvelle."

Quel est, notamment, le rapport du monde social et du monde vivant? Au sens le plus géné-: ral, le voici. Le social est un aspect du vivant. Les êtres organisés présentent à considérer deux sortes de relations: les relations internes entre les parties composantes d'un même organisme, les relations externes entre les divers organismes. Les premières forment l'objet de la biologie. Les secondes sont celui de la sociologie.

Nous aurons, bien éntendu, à revenir mainte fois sur cette distinction. Disons, dès à présent, ce qui nous paraît la constituer essentiellement. Les relations internes, organiques, sont en principe inconscientes. Au contraire, les relations externes, sociales, sont en principe conscientes. Les êtres vivants se font une certaine représentation les uns des autres et se guident dans leur activité extérieure par cette notion.

- Le principe ainsi posé va trouver immédiatement son application. Il nous permet en effet de comprendre que le domaine organique et le domaine social n'aient pas une égale extension. Le. domaine social n'est, sur aucun point, plus large que le domaine organique. I' n'existe de société. qu'entre les êtres vivants. Parler de sociétés d'astres ou de sociétés d'atomes, c'est user de métaphores. Mais, à l'inverse, le domaine social est, sur bien des points, moins large que le domaine 
organique. Ceei a besoin d'être quelque peu précisé.

D'abord, il y a tonte une catégorie d'êtres vivants qui ne forment pas de vraies sociétés : ce sont les végétaux. Sans doute, on a quelquefois soutenu le contraire. On a essayé de voir une société dans le groupement, soit de deux végétaux d'espèces différentes, soit de multiples végétaux de même espèce, soit encore de multiples végétaux d'espèces variées. Le premier cas est celui du champignon et de l'algue se juxtaposant pour constituer un lichén. Le second est celui des chênes, ou des pins; ou des sapins, dont l'agglomération forme une forêt. Le troisième se tire du second, si l'on réfléchit que, dans une forêt, il n'y a pas seulement des végétaux de l'espèce dominatrice qui lui donne son aspect extérieur et son nom, mais qu'il y entre aussi des végétaux de tout gente, de plus petite taille et subordonnés, qui. vivent à l'ombre des premiers. Un jardin pourrait être, encore mieux, donné comme le type d'une société végétale de ce troisième ordre.

Certes, il y a matière à des études botaniques intéressantes sur ces divers objets. Mais justement ce ne peuvent être que des études puremnt botaniques. Les relations des plantes voisines entre elles affectent évidemment chacune d'elles'et se répercutent sur leur vie organique. Seulement, le principe qui préside à ces relations est celui du voisinage dans l'espace, et ce voisinage s'explique par des causes ou physiques ou biologiques. De même qu'il existe une géographie worus 
botanique, étudiant l'action du sol et du climat sur les plantes, il peut y avoir une éthologie botanique, étudiant les actions des plantes les unes sur les autres. Mais parler d'une sociologie botanique serait, à tout le moins, prématuré.

Au-dessus des plantes, dans l'échelle des êtres organisés, viennent les animaux. Ici l'on ne peut contester qu'apparaissent des sociétés véritables. Le fait était reconnu de très longue date et il a provoqué de multiples et savantes recherches. Le livre justement réputé de M. Alfred Espinas sur Les sociétés animales en a donné une précieuse synthèse. Ce qui a été écrit depứs lors n'a guère ajouté que des détails. Pour notre part, nous ferons surtout remarquer que ces groupements ont le caractère proprement social chez les animaux intelligents et l'ont moins nettement chez les autres. Ainsi, bien que la division du travail soit poussée fort loin chez les siphonophores, on hésite à y voir des sociétés. On hésite également à le faire pour les ascidies composées, malgré leur remarquable coopération. Ces deux sortes d'êtres semblent moins des sociétés que des organismes complexes. Il n'en est plus de même pour les groupements d'hyménoptères (fourmilières ou ruches), pour les bandes de poissons, d'oiseaux ou de mammifères. Cette fois, tout le monde parle de sociétés. Sans doute, entre ce cas et le précédent, il y a une importante différence anatomique: c'est qu'ici on se trouve en présence d'organismes discrets, tandis que là on avait affaire à des organismes coalescents. Mais il y a en 
même temps, semble-t-il, une importante différence psychique. Ici, les individus ont, vraisemblablement, quelque représentation d'un but poursuiyi en commun. Là, vraisemblablement, ils n'en ont aucune. Concluons donc: la société apparaît là où l'intelligence s'affirme.

Arrivons à l'humanité. C'est évidemment chez elle que la sociabilité prend sa pleine extension. De toutes les sociétés existantes, les sociétés humaines sont, incomparablement, celles qui nous intéressent le plus. Et parmi elles, lesquelles doivent, avant toutes les autres, nous intéresser? Il existe à cet égard deux façons de penser opposées. La sociologie naissante, avec Auguste Comte, s'occupait surtout des groupes humains supérieurs. Le monde occidental avait presque seul du prix à ses yeux. La- sociologie contemporaine tend à faire exactement l'inverse: Pour 1'école d'Emile Durkheim, qui s'est inspirée en cela des grands anthropologistes et folkloristes anglais, ce sont les populations primitives qui doivent occuper le premier plan. Il ne manque pas d'arguments sérieux à l'appui de l'une et de l'autre conception. Durkheim estime qu'il faut voir les institutions à leur origine pour en comprendre le développement ultérieur. Cela est incontestable en effet. Mais Comte répondrait qu'il serait assez vain de se confiner dans la préhistoire, et que l'humanité demande à la sociologie de lui faire bien connaître son état présent, afin de préparer et d'organiser rationnellement son état à venir. Nous estimons que ce langage serait, lui 
aussi, bien fondé. La vérité nous paraît être que les deux thèses opposées sont, pour reprendre les termes de Leibniz, "vraies par ce qu'elles affirment, fausses par ce qu'elles nient "). I1 faut étudier à la fois les origines et l'état actuel des sociétés humaines. Mais, à notre sens, l'étude de l'état actuel est la plus fructueuse. La matière en est plus riche, plus complexe, plus haute. Elle est aussi d'un intérêt pratique plus direct. Elle prête en outre à une connaissance plus assurée. Car, d'abord, le présent peut être constaté, tandis que le passě ne peut être que restitué. Et surtout, le présent nous est directement intelligible, car ceux qui $y$ vivent ont des motifs d'agir et des façons de penser qui sont à peu près les nôtres; pour le passé au contraire, et surtout pour le passé très lointain, les mécanismes psychiques sont profondément différents de ceux que nous portons en nous. Au fond, c'est encore ici une considération psychique qui domine le débat. Les grandes sociétés contemporaines sont, pour la science, les plus instructives de toutes, surtout parce que ce sont celles dont nous pénétrons le mieux l'esprit. 


\section{CHAPITRE VII}

LE FAIT SOCIAL FONDAMENTAL.

Les relations entre les êtres vivants - spécialement entre les hommes - sont, comme nous venons de le voir, la matière de la sociologie. Mais cette formule n'a-t-elle point besoin d'être précisée? Ne pourrait-on, notamment, dégager un fait fondamental qui constituât l'essence ou la caractéristique de ces relations?

On l'a parfois tenté, et de là sont nés deux systèmes qui, depuis un quart de siècle environ, se disputent, principalement en France, la faveur des sociologues. L'un fut celui de Gabriel Tarde. A l'autre s'attache le nom d'Emile Durkheim. Essayons de caractériser brièvement l'attitude de ces deux penseurs sur la question qui vient d'être posée. Nous indiquerons ensuite notre conception personnelle.

- Le principe de la théorie de Tarde, c'est qu'il n'y a de société qu'entre êtres semblables. La similitude des assoaciés est à la fois la base, le 
moyen et la fin de la vie en commun. Car celle-ci suppose une certaine ressemblance initiale et elle s'en sert pour en réaliser une plus complète. Comment donc y arrive-t-elle? Par le mécanisme de l'imitation. Les êtres qui se reconnaissent analogues s'imitent entre eux. L'imitation est d'abord unilatérale : l'inférieur copie le supérieur. Elle devient ensuite réciproque: le supérieur emprunte quelque chose à son inférieur. Elle va du dedans au dehors, en ce sens que l'imitateur adopte l'idéal de son modèle avant d'en reproduire les actes. Telle sont les lois de l'imitation que Tarde a posées. Par le développement de ce processus, pense-t-il, les hommes arrivent au maximum possible de ressemblance. Les inventions de chacun d'eux se diffusent à travers tout le groupe. Les idées nouvelles, les façons de faire ingénieuses et utiles se propagent et se font admettre. Avec des inventions et des imitations on reconstruit intégralement 1'histoire humaine. "La société est un tissu dont l'invention est la trame et dont l'imitation est la chaîne ".

Telle est du moins la théorie de Tarde dans le premier et le plus important de ses ouvrages, Les lois de l'imitation. Dans un petit livre postérieur en date, où il condensa tout le développement de sa pensée, Les lois sociales, il a présenté les choses un peu autrement. Il distingue cette fois trois principaux modes d'action humaine: la répétition, l'opposition, l'adaptation. Mais, comme il le dit lui-même, la répétition, dans la société, c'est l'imitation; l'opposition, c'est une contre- 
imitation ; et l'adaptation, c'est encore, pour partie au moins, une sorte d'imitation. On retrouve donc le processus imitatif au fond de chacun de ces trois modes. La pensée de l'auteur, si sa forme n'est plus la même, n'a guère changé danș son fond. A toutes les dates, il a estimé que la relation fondamentale entre êtres humains est la relation imitative.

La theorie de Durkheim s'oppose à celle-là, mais non pas directement. Elle ne se place pas, en effet, exactement sur le même terrain. Tarde cherchait l'essence intime des relations sociales; Durkheim cherche seulement leur caractéristique extérieure. Le premier est un métaphysicien, le second entend être un pur homme de science. Ce que veut simplement Durkheim, c'est trouver un critère auquel on puisse, du dehors, reconnaître les phénomènes sociaux, en les distinguant des phénomènes purement individuels. Ce critère, selon lui, ce sera la contrainte. Est social le fait qui est accompli sous la pression de la société. Nos croyances, nos façons d'agir, ne sont pas notre œuvre propre. Nous les trouvons préconstituées dans notre milieu. Nous ne sommes pas libres 'd'en adopter d'autres, parce que ce serait nous mettre hors du groupe social. La religion, le droit, les mours, sont autant de formes de cette contrainte que le groupe fait peser sur nous. Son empreinte est mise sur presque tous nos actes, et c'est fort heureux, car par là nous nous relions à nos congénères et bénéficions de leur activité. Notre servitude, comme aurait dit Pas- 
cal, fait en même temps notre grandeur. La cité apparâ̂t ainsi, au sociologue contemporain, comme la divinité apparaissait au croyant d'autrefois. "In ea movemur, vivimus et sumus ". Sans" elle l'individu ne peut être ni expliqué ni même conçu.

Ces deux théories sont, à coup sûr, fort diver gentes. Dans le fait social, Tarde voyait la libre adhésion de l'être humain à un modèle voisin de lui. Durkheim y vit la coercition exercée par: le groupe sur les éléments qui le composent. Nous aurons à revenir, dans le chapitre suivant, sur la raison profonde de cette opposition. Mais ici nous devons signaler une similitude qui, malgré tout, rapproche ces deux auteurs, et que l'on. a généralement méconnue. C'est que l'un et l'au-: tre ont posé la question de la sorte: comment distinguer le social de l'individuel? Pour Tarde, le social, c'est l'imitation; 1'individuel, c'est l'in-' vention. Pour Durkheim, le social, c'est la règle d'action imposée par la collectivité ; l'individuel, c'est la modalité abandonnée à l'initiative de chacun. Pour ces deux auteurs, quoique selon des critères différents, il y a possibilité de distinguèr dans les actes humains des éléments sociaux et des éléments individuels.

En ce qui nous concerne, nous aurions des réserves à faire sur 1'une et l'autre de leurs théories. D'abord, leur opposition établit que chacune d'entre elles est incomplète. Quand les hommes agissent de même, c'est tantôt parce qu'ils s'imitent spontanément, tantôt parce que l'orga- 
nisation sociale les contraint à suivre une voie unique. Dans le premier cas, c'est Tarde qui a raison; dans le second, c'est Durkheim; mais aucun des deux ne rend compte de toutes les hypothèses. Et l'on constate qu'il en est certaines qui ne rentrent ni dans la théorie de l'un, ni dans celle de l'autre. Tel est le cas, lorsque des êtres éloignés dans l'espace ou dans le temps se trouvent agir de même façon, sous l'empire de facteurs physiques ou organiques semblables; sans qu'on puisse par conséquent invoquer, ni l'imitation, ni la contrainte sociale.

D'autre part, on peut se demander si la séparation que les deux auteurs tendent à établir entre l'individuel et le social est aussi radicale qu'elle le leur a paru. Sans doute, il est bon de distinguer, pour les besoins de l'étude, ces deux fractions. Mais, dans la réalité, l'écart est bien petit. Le social est fait d'éléments individuels et l'individuel est pénétré d'éléments sociaux. On passe par d'imperceptibles transitions de l'un à l'autre. Gabriel Tarde, qui avait à un haut degré le sentiment des nuances, s'en était bien aperçu. Et nous y insisterons encore plus que lui. Dans toute imitation, dirons-nous, il y a quelque chose d'ori-. ginal : car nul ne copie un modèle sans le déformer; à l'imitation se mêle donc sans cesse l'invention. Réciproquement, toute invention utilise un matériel préconstitué, suit une voie où des devanciers se sont engagés, renferme donc une certaine part d'imitation. Si l'on veut prendre maintenant l'autre langage, tout acte imposé par WORMS 
les règles sociales ne s'accomplit pourtant que suivant une modalité dépendant des moyens et souvent des caprices de l'individu. Et tout acte individuel doit compter, au moins comme limite, avec une règle sociale. Il y a, ainsi, une incessante pénétration réciproque des deux éléments. L'individuel ne se peut opposer au social.

Dès lors, la question de savoir comment le social doit se définir perd un peu de son importance. Nous ne croyons donc pas qu'il y ait lieu de chercher pour lui un critère différentiel. Nous voudrions plutôt en trouver une définition large, qui ne péchât point par défaut. C'est pourquoi nous proposerions la suivante. Est social, à nos yeux, tout ce qui suppose le concours d'une pluralité d'individus. Le concours n'est pas un simple contact, il implique une activité en commun, une coopération. Il y a concoúrs dès que deux individus, ou à plus forte raison plus de deux, pensent, parlent ou agissent ensemble. Le concours subsiste, lors même que de cette rencontre jailliraient certaines oppositions, car jusque dans ce cas on peut trouver une intention commune aux êtres en présence. Nous reconnaîtrons encore qu'il existe, lors même qu'on ne se trouverait directement en face que d'un seul être, dans le cas où celui-ci agirait nettement sous l'influence d'un ou de plusieurs hommes éloignés. En somme, la rencontre mentale des êtres, voilà pour nous le fait social originaire. Notre terminologie, nous le reconnaissons volontiers, n'est pas très explicative, mais elle ne vise pas à l'être. Elle 
constate simplement. Elle ne mutile paś le domaine social, et par suite elle ne le déforme pas. Au début d'une science concrète, on ne saurait poser une définition qui éclaire pleinement le défini. La logique est satisfaite si elle l'embrasse tout entier et ne peut s'appliquer qu'à lui, 


\section{CHAPITRE VIII}

RÉALITÉ DE LA SOCIÉTÉ.

Le débat entre Tarde et Durkheim, dont il vient d'être question, avait pris, il y a une vingtaine d'années environ, une ampleur singulière. Il passionnait les milieux intellectuels, en les divisant en deux camps. La raison en était sans doute qu'on apercevait, derrière les formules particulières à ces deux auteurs, deux principes généraux de philosophie sociale, qui se font antithèse depuis bien longtemps, et qu'on devinait, par delà ces principes, deux doctrines de pratique sociale, qui s'opposent depuis des siècles. Tarde était foncièrement individualiste, car il ne voyait partout que des hommes imitant les inventions de leurs semblables ou, plus rarement, inventant eux-mêmes. Durkheim, au contraire, voyait par-. tout la collectivité mettant sa griffe sur l'individu; il tenait pour une explication " collectiviste " de la vie sociale, s'il est permis de détour- 


\section{REALITÉ DE LA SOCIETÉ}

ner le mot "collectiviste " de son acception pratique pour l'introduire dans le domaine proprement scientifique. Or justement l'emploi nécessaire de ces deux mots - individualiste et collectiviste - montre le lien du problème scientifique que nous nous posońs avec les problèmes d'ordre pratique. En ce dèrnier ordre, les individualistes sont ceux qui veulent agrandir le champ d'action de la personne humaine; les collectivistes, ceux pour qui le groupe social doit passer avant.ses parties. Les premiers mettent en avant le principe de liberté; les seconds, le principe d'égalité. A coup sûr, le problème scientifique et le problème pratique sont profondément distincts. On peut avoir une opinion sur l'un et n'en avoir aucune sur l'autre. On peut même prendre sur l'un une attitude qui contraste avec celle que l'on adopte sur l'autre. Mais enfín, il y a quelque probabilité pour que la solution que l'on accepte dans l'un commande celle que l'on admettra sur l'autre. En logique, c'est le problème scientifique qui domine le problème pratique. En fait, c'est la préoccupation pratique qui inspire souvent la réponse à la question scientifique. On voit dès lors quels grands intérêts sont en jeu dans ce débat, et l'on comprend pourquoi il a tant divisé les esprits. Il est juste de dire, toutefois, que les deux protagonistes ne sont pas responsables de cette extension un peu abusive qu'il a prise pour beaucoup de gens. Aucun des deux ne mettait les questions d'application au premier plan,' et Durkheim les écarta toujours 
scrupuleuisement. Mais, en ces matières comme en d'autres, les chefs d'école ne sont pas maîtres d'arrêter les mouvements dont ils furent les initiateurs.

Nous voici donc arrivés en face du grand problème qui domine toutes les études sociales : est-ce l'idée de l'individu qui doit l'emporter, ou bien est-ce l'idée du groupe? Mais pour le philosophe, cette question de primauté suppose évidemment résolue une question préjudicielle: le groupe existe-t-il comme tel, et quel est son genre d'existence? Car, si les individus humains ont une réalité tangible, indiscutable, il n'en est pas de même des groupes par eux constitués. Il faut donc d'abord chercher si l'on a le droit de parler de ces groupes comme d'unités véritables, ayańt une valeur qui puisse être comparée et éventuellement opposée à celle des individus.

A notre avis, une distinction s'impose ici. La réalité du groupe social peut être admise. Mais on ne saurait reconnaître en lui une entité indépendante des êtres individuels. Nous nous séparons donc, à la fois, des partisans des deux thèses extrêmes. Aux purs individualistes, nous" disons : vous vous méprenez en niant toute existence autre que celle des hommes isolés. Et aux collectivistes, nous disons à leur tour: vous ne vous méprenez pas moins, en voyant dans la cité une sorte de divinité extérieure et antérieure aux simples mortels. Essayons de justifier brièvement notre attitude sur ces deux points.

Quand on dit que la collectivité est quelque 
chose de réel, il faut préciser de quelle collectivité il s'agit. Dans le monde végétal et animal, on n'hésite pas à reconnaître la réalité de l'espèce vivante. Il semble, à première vue, que l'espece humaine soit, à cet égard, comparable aux autres espèces animales. Cette idée a séduit de grands penseurs. Déjà Pascal avait envisagé l'humanité comme " un même homme qui subsiste toujours et apprend continuellement ". Auguste Comte, pareillement, a considéré l'humanité comme " le grand être " dans lequel les individus doivent voir " leur providence terrestre ". Cette conception si large semble aujourd'hui trop générale. L'unité humaine est loin d'être réalisée. Elle n'existe encore ni ethniquement, ni économiquement, ni moralement. Aux yeux du sociologue, l'espèce humaine se décompose en fractions très distinctes et souvent même ennemies. Cette décomposition peut se faire à divers points de -vue, sur lesquels nous reviendrons plus tard; les plus importants sont ceux de la race, de la langue, de la religion, de la nationalité. Pour ne parler ici que de ce dernier, les nations revendiquent jalousement leur indépendance réciproque. On peut dire que chacune d'entre elles $a$, en sociologie, la même importance au moins, que chacune des especes vivantes en biologie. Elle en a même une plus grande, car les nations sont moins nombreuses que les espèces. En termes plus précis, le concept social de nation est comparable au concept biologique d'espèce. C'est dire qu'il n'y a pas lieu de regarder, dans 
l'état présent des choses, l'espèce humaine commeun tout unique.

Mais, par là même, chaque nation tend à ressortir comme possédant ce caractère. C'est au groupe national qu'on est porté à attribuer l'unité véritable et la réalité. Par bien des côtés, en effet, les nations semblent être des individus. Chacune d'elles a un nom, une histoire, des traits mentaux et matériels distinctifs. Chacune forme un Etat, avec un. gouvernement qui en est la repré- sentation concrète. Chacune possède, en droit international, les attributs de la personnalité morale. Dans leurs rapports ávec les individus qui' les composent, deux grands faits apparaissent immédiatement. D'une part, la nation forme l'individu, lui donne sa langue, ses idées, ses mœurs; elle lui assigne sa place par le jeu de la division du travail, et assure ainsi sa subsistance, en même temps qu'elle détermine et limite son action. D'autre part, la nation survit à l'individu : quand il disparaît, elle demeure; il ne dépasse pas un siècle, elle peut être millénaire ou multi-millénaire; comme l'individu se rajeunit par l'élimination de ses cellules anciennes et la formation de cellules nouvelles, la nation se rajeunit par la disparition des êtres vieillis et l'apparition d'êtres nouveaux. Et, dans un ordre d'idées différent, ne voit-on pas les nations se comporter les unes envers les autres comme de véritables individus? La guerre ne les dresse-t-elles pas tout entières, frémissantes et armées, dans un grondement de colère furieux? Tous les membres d'une 
nation, à ce moment, ne font qu'un. Tous se portent d'un même élan contre l'ennemi commun. Tous ont les mêmes soucis dominants, tous n'ont vraiment 'qu'une seule âme. En ces instants tragiques et significatifs, l'individu sacrifie de bon cœur son temps, ses intérêts, sa vie, à l'Etat dont il relève, sans hésitation et sans réserves. Il ne combat pas pour ses biens, pour son foyer, pour telle personne qui lui est chère. Il combat pour sa patrie, dans laquelle il ne distingue plus. S'immolerait-il à elle, s'il ne croyait à sa réalité supérieure?

Tout cela est exact, à coup sûr, et il serait absurde de le méconnaître ou de l'oublier. Mais il y a aussi 1'autre face de la question. Car les choses humaines sont ainsi faites, qu'elles ont toujours au moins deux aspects différents et d'ordinaire opposés. De même que tout tissu a son endroit et son envers, que toute'médaille a - un avers et un revers, de même toute œuvre humaine, y compris la plus grande de ces ouvres, la société, a ses deux faces contraires et pourtant nécessairement liées. Elles sont complémentaires l'une de l'autre, et aucune ne se suffit à elle seule. Hégel passait pour admettre l'identité des contradictoires; sans aller jusque-là, nous admettons du moins, pour notre part, la coexistence et la liaison des contraires. Nous, venons de montrer, la réalité de l'être collectif. Hâtons-nous d'ajouter que cette réalité n'a pourtant rien qui le sépare des êtres individuels et qui l'en rende indépendant. L'Etat n'est point, WORMS 
comme l'ont voulu quelques nébuleux écrivains allemands, une entité mystérieuse douée d'une existence propre (" selbstständig ") qui subsiste par soi-même et se passe du concours des hommes. Cette conception mystique se dissipe très vite à la claire lumière de l'esprit français. Et pourtant il importe de mettre en garde contre elle, car certaines expressions contestables de Durkheim donneraient à croire que, mêtme en notre pays, cette terminologie s'est fait partiellement? recevoir. Non, une société n'est aucunement un être à part des individus, Quand on parle du corps : social, c'est l'ensemble des citoyens qu'on entend désigner. Et, quand on parle de l'esprit collectif, on songe aux esprits individuels, envisagés dans leur fond commun, dans les idées et les sentiments `qui les réunissent. Le groupe ne vit que par ses membres et en eux. Par suite, il ne peut vivre que pour eux. - Lorsqu'on dit que le citoyen doit vivre pour 1'Etat, cela a deux sens possibles. Ou bien l'on entend qu'il doit travailler pour l'ensemble de ses semblables, et alors c'est un précepte de morale, excellent à coup sûr, mais qui gagnerait à être formulé d'une manière un peu différente. Ou bien '1'on entend qu'il doit travailler pour les gouvernants; mais cette idée, admissible dans un régime monarchique, où le souverain incarne la patrie, ne l'est plus dans un pays républicain. Ici, au contraire, on doit dire que c'est aux gouvernants à travailler pour les gouvernés; que les premiers ne tirent leur raison d'être que des besoins des seconds ; que les avan- 
tages dont ils bénéficient ont leur principe et leur limite dans les intérêts de la masse de leurs concitoyens. Ainsi les droits de chaque individu apparaissent en face des droits de l'Etat et des représentants de ce dernier. Ainsi ils se montrent comme corrélatifs les uns des autres, comme se faisant réciproquement contre-partie et contrepoids, ou plus exactement, comme tirant leur origine de la même source et comme n'étant, eux aussi, que les deux faces d'un même droit.

Le droit, ici, sort directement du fait. S'il y a vis-à-vis l'un de l'autre deux droits antithétiques et inséparables, c'est parce qu'il y a dans la même attitude deux réalités corrélatives: les individus et le groupe. Le second, nous l'avons dit, n'est pas une pure abstraction. Mais il n'existe pour-, tant que par les premiers. Les deux propositions sont vraies à la fois. Comment peuvent-elles l'être? On ne voit pour cela qu'un moyen. C'est que le groupe consiste dans une organisation des irdividus, organisation qui lui donne une matière et leur donne une forme. Une semblable conception est peut-être de nature à concilier bien des thèses oposées. Mais on peut nous demander de la compléter, et c'est ce que nous voudrions essayer. 


\section{CHAPITRE IX}

CONTRAT SOCIAL OU ORGANISME SOCIAL.

En quel sens le groupe est-il une organisation des individus? Bien des réponses ont été faites déjà à cette question. Celles qui sont le plus en faveur aujourd'hui se rattachent à l'un ou à l'autre des systèmes qu'on se plaît d'ordinaire à opposer sous les noms quelque peu barbares de contractualisme et d'organicisme. Nous croyons, pour notre part, à la possibilité de les concilier.

Le contractualisme a été, à la date où il naquit, un incontestable progrès sur les théories antérieures. Celles-ci faisaient reposer le lien social sur le seul principe d'autorité, qu'elles attribuassent 1'autorité à la force physique, à l'hérédité, ou à la manifestation d'une volonté supra-sensible. La théorie du contrat social, elle, a vu dans ce lien la manifestation de la volonté des associés eux-mêmes. Elle a estimé que la vie sociale dérivait du concours libre et spontané des intéressés. C'est donc de l'autonomie de la personne humaine qu'elle est partie, et pour elle l'existence collec- 
tive a pour but d'assurer et de renforcer cette autonomie. Cette doctrine a d'abord été esquissée par Spinoza. Elle a été ensuite exprimée avec éloquence par Jean-Jacques Rousseau. On la retrouvera chez Kant. C'est elle qui a inspiré la Déclaration des Droits de l'homme et du citoyen. C'est elle qui domine encore la constitution de notre République et, consciemment ou non, le libéralisme contemporain.

Comme principe d'action, elle peut paraître des plus satisfaisantes. Il semble moins facile de la justifier comme explication de l'histoire. Au temps où elle fut formulée, on n'était pas très exigeant à cet égard. Déjà pourtant Rousseau ne la présentait qu'avec un caractère hypothétique. I1 " supposait ", dans les premiers chapitres de son Contrat Social, qu'il avait pu au début de l'humanité exister un " état de nature ", où les hommes auraient vécu isolés; il se gardait d'affirmer cette existence. De nos jours, elle semble une proposition insoutenable. Personne ne croit plus (si quelqu'un 1'a jamais cru) que les individus aient commencé par la solitude. La société nous apparaît comme un fait originaire, et non pas comme un fait dérivé.

Tel est justement le principe sur lequel s'appuie aujourd'hui la théorie connue sous la dénomination d'organicisme. A la vérité, celle-ci est fort ancienne: l'antiquité et le moyen-âge l'avaient au moins entrevue ( $\mathrm{r}$ ). Mais au $\mathbf{X I X}^{\circ}$ siè-

(1) Voir Ludwig Stein, Les conceptions mécaniques et 
cle elle a pris un aspect beaucoup plus scientifique. Auguste Comte avait parlé de " l'organisme social », tout en faisant des réserves sur cette expression (1). Herbert Spencer 1'a prise beaucoup plus à la lettre. La seconde partie de ses Principes de sociologie est consacré à en établir la valeur. La même conception s'est fait également admettre par des écrivains d'autres nationalités, spécialement par les sociologues russes Jacques Novicow (2) et Paul de Lilienfeld (3). Mais, contrairement à ce que 1'on dit d'ordinaire, un éminent auteur allemand, Albert Schæffle, ne 1'a reçue, si l'on peut ainsi dire, que " à corrections "': car il a écrit expressément, dès le début de son principal ouvrage (4), que " la société est une organisation, non un organisme ". En France, l'organicisme a surtout eu la bonne fortune de se mêler au solidarisme, et il en semble aujourd'hui - quoique peut-être sans raisons logiques suffisantes - difficilement séparable (5).

Pour les partisans de cette doctrine, 1'humanité

organiques de l'Etat; articles publiés dans la Revue internationale de sociologie, I9I I.

(1) Système de politique positive, tome II, chapitre V.

(2) Conscience et volonté sociales.

(3) Pensées sur la science sociale de l'avenir, parues en russe et en allemand. La Pathologie sociale, parue en français.

(4) Bau und Leben des sozialen Korpers.

(5) Voir notamment le livre de M, Léon Bourgeois : Solídarité. 
n'a pas eu à imaginer un jour la forme sociale. Elle 1'a connue de tout temps. L'homme est par essence, suivant l'expression célèbre d'Aristote, un animal sociable. La société est son milieu naturel et l'a toujours été. Il est simplement une des cellules de l'organisme social. Celles-ci ont entre elles des rapports analogues à ceux qu'ont les cellules vivantes au sein de l'organisme individuel. Elles s'agencent, comme celles-ci, en tissus et en systèmes, en organes et en appareils. Comme . les organismes individuels, les organismes sociaux ont une vie de nutrition, une vie de reproduction, une vie de relation. Ils obéissent, comme eux, aux grands principes biologiques d'adaptation, d'hérédité, de sélection. Ils sont, comme eux, soumis à des maladies et à des crises, et connaissent pareillement les phases successives de la naissance, de la croissance, de la maturité, du déclin et de la mort. Ainsi la comparaison peut se poursuivre, tant entre les éléments composants des deux sortes d'agrégats, qu'entre ces agrégats eux-mêmes.

Que penser d'une semblable comparaison? On doit reconnaître, croyons-nous, que dans les grandes lignes elle présente certains traits exacts. Par exemple, la division du travail existe certainement, tant entre les parties du corps vivant qu'entre celles de la société. D'elle découle 1'échange des services entre les éléments associés. Cet échange crée entre ces parties une solidarité obligatoire. Et c'est celle-ci qui plus tard, au sein de la société, devient réfléchie et voulue, et 
peut ainsi former une des bases de la morale. Après ces analogies d'ordre général, on en a relevé de plus particulières. Un certain nombre de faits de la vie sociale rappellent d'une façon: assez frappante divers phénomènes de la vie organique. Ainsi la circulation des richesses n'est pas sans ressemblances avec la circulation du sang. Spencer a esquissé un curieux parallèle entre les fils télégraphiques et les filets nerveux. Mais, quelque suggestifs que puissent être de sembla-: bles rapprochements; on voit déjà, par ce dernier exemple, qu'il faut savoir s'arrêter à temps dans cette voie, si l'on ne veut transformer une comparaison scientifique en une pure et simple métaphore.

Ce que l'on peut dire dans l'ensemble à cet égard, c'est que les processus sociaux ont leur base et leurs modèle lointain et incomplet dans les processus organiques. Les sociétés, comme les organismes, font partie de la nature vivante. Les unes comme les autres sont donc forcément soumises à de mêmes lois générales. Les modes d'agencement et d'action qu'étudie la biologie se retrouvent, par suite, au sein des objets sur lesquels porte 1'investigation de la sociologie. Mais il y sont singulièrement amplifiés et compliqués. D'innombrables éléments nouveaux s'y introduisent, sous l'action des idées et des volontés humaines. Et celles-ci sont, on le sait de reste, de toutes les forces de la nature, les plus complexes, les plus diverses dans l'espace, les plus changeantes dans le temps. Voilà pourquoi, par exemple, tan- 
dis que la circulation du sang dans le corps humain peut se traduire par un schéma simple et invariable, la circulation des richesses dans les sociétés humaines ne saurait être exprimée graphiquement de la sorte. Les figures qu'on en pourrait dresser devraient être surchargées de traits entrecroisés ; chacune d'elles ne vaudrait, tout au plus, que pour une nation; chacune devrait être retouchée au moins tous les dix ans. De telles difficultés expliquent que, le plus souvent, on ait renoncé à ce genre de représentations. Et elles mettent en lumière ce fait que l'économie politique est une science moins précise et moins susceptible d'achèvement que la physiologie animale.

L'homme, dans ses créations propres, s'inspire de la nature, mais il sait la dépasser. Nos outils, nos appareils copient sans doute certaines formes que nous avons trouvées dans notre milieu, mais pour une part ils sont le produit de notre invention. Ainsi la fourchette imite la main, ainsi l'aile de l'aéroplane imite l'aile de l'oiseau, mais en les perfectionnant; et entre ces deux inventions, quel écart dans l'ingéniosifé et dans la portée, mais aussi combien de siècles d'efforts! Ce que nous disons là des œuvres humaines particulières peut se dire de l'œuvre humaine la plus générale, l'organisation de la vie collective. Elle est partie d'une base étroite et simple, que la nature fournissait. Elle s'est élevée, à travers les âges, par un effort continu, par une accumulation de découvertes, pour former une superstructure 
d'une hauteur et d'une largeur extrêmes. Seulement, l'éclat de cet édifice en fait deviner la fragilité.

En somme, il reste quelque chose d'organique dans cet édifice. Mais cet élément organique a été recouvert, en même temps qu'utilisé et développé, par des rapports mentaux successifs et innombrables. Suivant une frappante expression de M. Espinas, la société est en quelque sorte " un organisme d'idées ". Et déjà Spencer lui reconnaissait ce caractère, à la vérité indéniable. Car il parlait, d'ordinaire, moins de l'organisme que de "l'hyper-organisme " social.

Par là, nous arrivons à apercevoir la conciliation possible, que nous annoncions au début de ce chapitre, entre la théorie organique et la théorie contractuelle des sociétés. L'une et l'autre ont un élément exact; chacune traduit une face de la réalité ; ces deux faces ont seulement besoin d'être juxtaposées, ou plutôt superposées. La théorie organique fait comprendre le point de départ des sociétés; la théorie contractuelle, leur point. d'aboutissement. Les sociétés naissent à la façon des organismes et s'agencent d'abord suivant les mêmes lois que ceux-ci. Eles progressent ensuite par un mode plus spécifiquement humain, en se dirigeant vers un idéal conçu par l'esprit: idéal de justice, de paix, de liberté, de lumière. Elles tendent, par là, à réaliser entre leurs membres une égalité et une solidarité contractuelles. Du monde organique on passe ainsi au monde social, sans secousses et sans interruption, par.l'intermédiaire 
du monde mental. La société, telle du moins que notre âge la conçoit et la veut, sera véritablement, comme Alfred Fouillée l'a si bien dit ( 1 ), un " organisme contractuel ". L'idée d'évolution rapproche les deux termes de la formule et en fait comprendre l'unité (2).

(1) Dans La science sociale contemporaine.

(2) Nous avions adopté les principes de la théorie organiciste et les avions même développés dans un volume paru en 1896 et intitulé Organisme et société. L'étude, l'expérience et la réflexion nous ont appris ultérieurement à restreindre l'adhésion que nous leur avions d'abord don. née, ou plutôt à faire place, à côté d'eux, à des principes assez distincts. Aussi ne les trouvera-t-on plus admis que sous une forme atténuée dans nos écrits ultérieurs : les trois volumes de la Philosophie des sciences sociales (1903-07 ; seconde édition, 1913-20), Les principes biologiques de l'évolution sociale (1910), et les pages du présent ouvrage qui vont suivre. Nous espérons, du reste, pouvoir donner prochainement une deuxième édition d'Organisme et société où seraient distingués, dans cette théorie, ce qui est caduc et ce qui demeure. 


\section{CHAPITRE $\mathrm{X}$}

D̈IVISION DE LA SOCIOLOGIE.

Si les sociétés sont des réalités, dans une certaine mesure comparables aux organismes, les études qui portent sur elles doivent elles-mềmes se rapprocher sur certains points des sciences de la nature. Celles-ci sont actuellement fort avancées. On doit donc pouvoir en tirer quelque lumière pour la projeter sur celles-là. Nous croyóns que tel est le cas en effet. Et nous pensons que cette utilisation peut se faire de deux manières: D'une part, des considérations de cet ordre peuvent servir à préciser la division de la sociologie. D'autre part, des vues du même genre peuvent servir à en préciser la méthode. Dans le présent chapitre, nous n'aborderons que la première de ces deux questions.

Le problème de la division de la sociologie a été posé par le fondateur de cette science, Augusté Comte. Pour le résoudre, il s'inspira d'un rapprochement entre la sociologie et la mécanique. Et 
cela était assez normal, puisque pour lui la science nouvelle était une " physique sociale ". Il proposa donc de la diviser en deux parties: la statique sociale et la dynamique sociale. Dans son Cours de philosophie positive, dont la sociologie occupe trois volumes. sur six, il consacre à la statique le tome IV, à la dynamique les tomes $\mathrm{V}$ et VI. Et dans son Système de politique positive, en quatre volumes, la statique occupe-le tome II et la dynamique le tome III. Pour lui, la statique est la théorie de l'ordre humain et la dynamique est la théorie du progrès humain. La première étudie les éléments stables de la société et la seconde envisage ses éléments changeants. Il croit de très bonne foi que cette division est comparable à celle de la mécanique et aussi à cellè de la biologie. Car la statique, dit-il, correspond à l'anatomie, et la dynamique à la physiologie. Sur ces derniers points, son erreur est palpable, et nous aurons à y revenir dans un instant. Mais il n'est que juste de montrer d'abord ce qu'il y avait d'exact et de suggestif dans ses vues.

L'idée intéressante du système, et qu'on doit en retenir encore aujourd'hui, c'est que la société peut être étudiée, soit au repos, c'est-à-dire dans son aspect à un moment donné, soit en mouvement, c'est-à-dire dans la série des formes qu'elle revêt aux périodes successives. Ainsi, veut-on décrire la société française? ou, pour prendre un objet ' plus limité et plus saisissable, veut-on décrire tel de ses éléments régionaux, par exemple la Normandie ou la Gascogne, ou encore tel 
de ses éléments fonctionnels, par exemple la magistrature ou le service de l'enseignement? On pourra prendre cet objet, vaste ou limité, dans son état présent et le faire connaître dans tout son détail; on aura alors fait de la statique sociale.' On pourra aussi, à l'opposé, envisager le même objet dans son devenir historique, commencer par ses origines et descendre ensuite le cours des temps pour arriver jusqu'à l'état de choses actuel; on aura fait alors de la dynamique sociale. Les deux façons d'opérer sont également légitimes. Toutes deux ont leurs avantages et, naturellement aussi, leurs inconvénients. Aucune d'entre elles ne donne la vérité totale. Mais elles se complètent parfaitement l'une l'autre. Elles doivent donc être associées, ou plutôt employées tour à tour. Auguste Comte l'a bien vu, et il est à son honneur. de l'avoir montré.

Malheureusement, sur presque tout le reste, nous devrons faire d'expresses réserves. I1 semble d'abord qu'Auguste Comte ait accordé à la statique trop d'importance par rapport à la dynamique. Il s'exprime comme s'il existait un ordre immuable des sociétés, une base véritablement fixe au milieu de leur mouvement incessant. Or c'est ce qui est extrêmement douteux. Pour les sociologues contemporains, l'évolution est le fait capital; toute la vie sociale change à chaque instant; l'arrêt y est un fait exceptionnel. Ou plutôt même, à vrai dire, il n'est qu'une apparence. Nous disons qu'il y a arrêt lorsque le mouvement ne frappe pas nos sens ou notre esprit. Mais ce n'est 
là qu'une impression subjective, ne correspondant pas à la réalité, où le mouvement ne s'arrête jamais. - Si cette vue est exacté, la dynamique seule atteindrait le fond des choses. La statique ne serait qu'un commode procédé de l'esprit. Avec celle-ci, on fixerait l'imagé d'un état social qui, un moment auparavant, était autre, et qui, un moment après, sera tout autre de nouveau. Une telle image ne saurait avoir que la valeur d'un " instantané ". Elle peut être exacte, sans doute, et à ce titre instructive. Mais le renseignement qu'elle fournit n'a qu'une portée très limitée. Il est purement provisoire. La science ne saurait, en aucun cas, s'en contenter.

Ce n'est pas tout. A la division de Comte, on peut reprocher d'autres erreurs. La première est une vicieuse terminologie, qui provient d'un conception inexacte. Comte veut comparer la sociologie à la mécanique, et c'est pourquoi il y distingue une statique et une dynamique. Mais la mécanique, elle, se divise en trois parties : la statique, la dynamique et la cinématique. C'est la cinématique qui y forme l'étude des mouvements, ce n'est pas la dynamique. Comte n'ignorait évidemment pas cette notion élémentaire, étant luimême mathématicien, et exerçant à l'Ecole Polytechnique les fonctions de répétiteur d'analyse et de mécanique. On s'étonne donc qu'il n'ait pas cru devoir appeler cinématique sociale l'étude des mouvements sociaux, et qu'il ait donné à cette étude le nom de dynamique sociale, sans d'ailleurs s'expliquer sur cette méconnaissance des 
termes reçus. La conséquence en a été, qu'il a ramené à deux les trois éléments de la mécanique sociale, et qu'il a apporté dans cette science extrêmement complexe une réduction arbitraire, que la science pourtant plus simple de la mécanique physique repousse. Mais, s'il a fait ainsi, c'est en raison d'une autre méprise qu'il, commettait, et sur laquelle il nous faut maintenant nous expliquer.

Cette seconde erreur consiste dans une assimilation vicieuse entre la division générale de la mécanique et celle de la biologie. Cette dernière se divise en anatomie et en physiologie. Or Comte estime que l'anatomie correspond à la statique et la physiologie à la dynamique. Mais, avec sa conception de la dynamique, c'est inexact. L'anatomie est l'étude des organes, la physiologie est l'étude des fonctions. La' statique est pour Comte 1'étude du repos, et la dynamique celle du mouvement. Il a dit que l'anatomie étudie les organes quand ils sont au repos, et que la physiologie les envisage quand ils se mettent en mouvement, c'est-à-dire quand ils fonctionnent. Sans doute; mais ce n'est là qu'une première vue de la question. Au sens le plus profond du mot, le mouvement des organes n'est pas le simple exercice normal de leurs fonctions, c'est leur transforma tion par l'effet de ce fonctionnement lui-même: - c'est, en un mot, leur évolution. L'étude de cette évolution constitue la vraie dynamique des corps vivants, au sens où Comte prend le terme de dynamique, c'est-à-dire au sens de cinématique. Quant 
à la physiologie, elle 'n'est pas, en un certain sens, moins statique que l'anatohie, car elle étudie les fonction̉s à un moment donné, tout comme 1'anatomie étudie les organes à un instant déterminé (1).

Poúrquoi Comte, qui était très versé en biologie, n'a-t-il point fait cette analyse, en somme assez simple? Pourquoi a-t-i1' confondu le fonctionnement, fait statique, et la transformation, fait (dans sa terminologie) dynamique? C'est, d'abord, parce que ces deux phénomènes sont liés dans la nature. La transformation des êtres ré-. sulte de leur fonctionnement. Le jeu de leurs organes explique leur croissance et leur déclin. Mais il faut se garder d'absorber l'effet dans la cause. Si Comte 1'a fait, c'est, en seqond lieu, parce qu'il n'avait pas la notion d'évolution organique telle que nous la possédons actuellement. I1 connaissait Lamarck; sans doute, et même il l'estimait fort, car il l'appelle toujours " l'illustre Lamarck "); mais il n'avait pas adhéré à sa doctrine, la voyant repoussée par toute la science de son temps. Quant à Darwin, il est à peine besoin de dire qu'il n'a pu le connaître, puisqu'il mourut deux ahs avant la publication de L'origine des espèces. Il n'était donc pas évolutionniste, et par suite son attention n'était pas appelée sur les phénomènes de transformation dans lesquels il

(I) Nous nous sommes eflorcé d'élucider toute cette question dans notre livre sur l'Objet des sciences sociales, au chapitre $\mathrm{X}$.

worms 
eût dû voir la vraie matière de ce qu'il nommait. la dynamiquè organique.

Et pourtant, s'il ignorait l'évolution des especes, il n'a évidemment pas pu ignorer l'évolution des individus. Il savait, comme chacun, qu'un organisme passe par des phases successives áppelées naissance, adolescence, maturité, sénescence, disparition. Ces transformations organiques, il a senti à un certain moment qu'elles ne sont pas identiques au simple fonctionnement des organes. Et c'est alors que, pour l'expliquer, i1 a tenté, à une date assez tardive, d'introduire une distinction entre l'existence et la vie (I). Plus tard son principal disciple, Littré, la reprendra en distinguant une physiologie d'entretien et une physiologie de croissance. Mais ce sont là des concessions insuffisantes, et il faut davantage.

Ce qu'il faut, à notre avis, c'est suivre la division tripartite de la mécanique dans les sciences plus complexes: biologie et sociologie. En biolo-. gie, si l'anatomie correspond. à la statique, la physiologie correspondra à la dynamique, et l'étude de l'évolution à la cinématique. Nous ne tenons pas d'une façon absolue à ces correspondances, mais du moins nous semblent-elles plus correctes que celles que Comte établissait. En sociologie, on pourra procéder comme en biologie.

(I) Dans le Système de politique positive, tome- II, chapitre VI. Il y écrit notamment : " la vie de chaque être. consiste dans les modifications qu'éprouve son existence ». 
Sans se faire illusion sur la portée du rapprochement entre les organismes et les sociétés, on devra étudier, dans celles-ci comme dans ceux-là : $\mathbf{I}^{\circ}$ les éléments constituants et leur agencemènt, c'està-dire les structures sociales, les organes sociaux ; $2^{\circ}$ le fonctionnement de ces éléments et de ces organes; $3^{\circ}$ leur évolution, c'est-à-dire à la fois la transformation des structures et celle des fonctions correspondantes. On pourra donc distinguer dans la sociologie trois parties, que l'on nommera, pour reprendre les termes usités en biologie: lianatomie sociale, la physiologie sociale, l'ontogénétique sociale ( $\mathrm{I}$ ). On pourra aussi les appeler, si l'on préfère les expressions usitées en mécanique: la statique sociale, la dynamique sociale, la cinématique sociale. Mais nous avouons que chacune de ces terminologies a ses inconvénients, parce qu'elle rapproche trop la sociologie d'une science plus simple, et tendrait à faire croire qu'elle s'y absorbe presque. Noús préférons donc dire, en termes moins savants peut-être, mais aussi moins contestables, que la sociologie a trois objets d'étude: les structures sociales, les fonctions sociales, l'évolution sociale.

(I) On sait que, sous le nom d'entogénèse, Ernest Hæckel a proposé de désigner tout ce qui se rattache au développement de l'organisme. 


\section{CHAPITRE XI}

LES ÉLÉMENTS SOCIAUX.

La première question que le sociologue ait à se poser, en présence d'un groupe social déterminé dont il veut faire 1'étude, est de savoir quels sont les éléments de ce groupe et comment ils sont agencés.

Parmi ces éléments, deux grandes catégories doivent être distinguées. Ceux auxquels on songe tout d'abord, ce sont les êtres humains compris dans le groupe envisagé. Mais à côté d'eux, il y a des choses sur lesquelles s'exerce leur action. Celles-ci n'appartiennent pas moins qu'eux à la société. Bỉen que leur rôle ne soit pas du même ordre, bien qu'il soit d'ordinaire passif (pas toujours, néanmoins), elles sont indispensables à la vie collective. Sans elles, les humains ne sauraient subsister. Elles ont donc leur place marquée dans les préoccupations du sociologue. Et c'est d'elles que nous allons parler tout d'abord, pour réser- 
ver ensuite, comme il est juste, nos plus longues explications aux hommes eux-mêmes.

I. - Les éléments non-humains de la société, les choses, se divisent, pour la sociologie, en deux classes. Les uns sont des produits de la nature; les autres sont des produit de l'art humain.

Voici une simple énumération de ceux qui dérivent de la nature seule. C'est, d'abord, le sol, emplacement indispensable à toute activité humaine. Sa configuration, particulièrement aux points de vue orographique et hydrographique, peut avoir une influence déterminante sur cette activité. - C'est, en second lieu, le sous-sol. Par ses richesses minérales, par les aliments qu'il fournit aux plantes, il domine l'industrie et l'agriculture. - C'est, en troisième lieu, ce que, par antithèse ou plutôt par symétrie, on pourrait nommer le "sur-sol ". Nous voulons désigner par là 1'atmosphère, y compris le climat, les météores, les forces physiques. Et nous y comprenons aussi les espèces végétales et animales que porte le sol. Tous ces éléments agissent grandement sur l'homme; mais l'homme, à son tour, sait les utiliser à ses fins et les faire entrer comme parties composantes dans ses œuvres.

C'est de là que naissent, précisément, les choses de la seconde classe, nous voulons dire les produits de l'art. L'art, au sens où nous prenons ici cette expression, est bien, suivant le mot de WORMS 
Bacon, "1'homme ajouté à la nature ". Car, si la nature fournit la matière de ses produits, c'est 1'homme qui leur donne la forme. Ces produits, ce sont les objets servant à satisfaire nos principaux besoins: les alimenț préparés, les vêtements, les habitations et les meubles. Ce sont encore les objets de luxe, tels que les parures, "ce superflu, chose si nécessaire " aux yeux de la majeure partie de 1'humanité. Ce sont aussi les armes. Ce sont enfin iles outils destinés à la fabrication de tous les objets précédents.

De l'unè de ces deux classes à l'autre, on passe par des transitions insensibles. Ainsi un fruit, tant qu'il reste sur l'arbre qui l'a produit, est'de la première classe. Mais quand il est cueilli, il passe dans la seconde, surtout s'il est immédia tement comestible. Il faut donc se garder d'exar. gérer la portée, et surtout la rigidité, de cette classification. Ici comme presque partout, 1'esprit est obligé, pour mettre quelque ordre dans son cont tenu, d'établir entre les choses, ou plutôt entre les idées qu'il s'en fait, des distinctions que la: réalité ne connaît point.

On ne saurait oublier de faire leur place, en sociologie, aux éléments non-humains de la société. On est parfois, au.contraite, tenté de la leur faire un peu trop grande. Certains syste tèmes ont été édifiés, qui sans doute l'exagèrent. Tel fut celui d'Edmond Demolins, pour lequel les caractéristiques de 1'homme viennent de son alimentation, et les traits distinctifs de chaque raced de la toute qu'elle a suivie dans ses migrations 
anciennes ( $\mathrm{I}$ ). Tel fut encore, dans un esprit assez différent, celui de Karl Marx, suivant lequel l'outillage de la production économique est, de tous les facteurs sociaux, le plus important, car " le mode de production de la vie matérielle domine en général toute la vie politique, intellectuelle et sociale " (2.). Ce sont là des affirmations outrancières, dont les esprits avertis se gardent d'ordinaire actuellement (3). Elles provenaient pourtant, il faut le réconnaître, d'une idée en soi-même exacte : la nécessité-des choses pour les hommes.

II. - Quelle que soit l'importance des éléments non-humains, celle des éléments humains nous paraît encore plus grande dans la structure sociale. La description de ces éléments relève à la fois des sciences naturelles et des sciences sociales proprement dites. Les premières envisagent 1'homme dans les caractères qui sont communs à toute l'espèce. Les secondes l'envisagent dans ceux qui sont spéciaux à ses diverses fractions. Ainsi 1'anatomie décrit la composition générale du corps humain; la démographie et l'ethnographie décrivent la composition particulière de chaque population et de chaque race. Pour compren-

(I) Voir ses livres : Les Franf̧ais d'aujourd'hui ; Les grandes routes des peuples.

(2) Le capital.

(3) Voir notamment Jean Brunhes, La géographie humaine. 
dre comment elles opèrent et comment la sociologie utilise leurs données, il faut se fixer sur la façon dont les hommes s'agrègent. Demandons-? nous donc comment les structures sociales résultent de l'agencement des éléments humains de la société.

L'observation montre que cinq modes au moins de groupement peuvent 'être relevés parmi ces éléments. Le plus anciennement connu, celui qui forme la base des sociétés primitives, c'est le groupement par parenté. Les êtres qu'unit le lien du sang sont les premiers qui restent adhérents les uns aux autres. Ce lien, du reste, peut être compris de bien des manières. On a cru très longtemps que la famille patriarcale, celle qui repose sur la parenté masculine, était la plus ancienne de toutes. Les exemples de Rome, de la Grèce, de 1'Inde, de la Judée, venaient à l'appui de cette conception. Mais, depuis un demi-siècle, les idées ont changé. Les travaux de Bachofen, de Mac Lennan, de Lewis Morgan et de leurs nombreux émules ont mis en lumière l'existence et 1'ancienneté de la famille matriarcale, dont la mère est le centre. Il semble que dans les sociétés primitives, ou du moins dans un grand nombre d'entre elles, aient prévalu des principes extrêmement contraires à ceux qui nous paraissent si " naturelš " aujourd'hui : l'exogamie obligatoire, l'union matrimoniale par classes, impliquant à la fois la polygamie et la polyandrie générales; l'ignorance de la paternité, l'absence de foyer 
individuel (I). Sans vouloir discuter ici la place de semblables. organisations dans le temps et dans l'espace - laquelle pourrait être plus restreinte qu'on ne le suppose souvent - bornonsnous à constater que ces recherches ont élargi les notions courantes sur le principe originaire de la famille, mais n'ont rien changé à la constatation faite il y a un instant par nous : à savoir, que le premier groupement humain a été la famille, quelqu'ait été le mode d'organisation de celle-ci, patriarcal ou matriarcal.

Après ce premier type social, s'en est constitué un second, fondé, non plus sur la parenté, mais sur le voisinage. Les diverses familles, venues en contact, se sont plus ou moins fusionnées. Des agglomérations plus vastes se sont établies. Les hommes s'y sont sentis unis, par le seul fait qu'ils étaient rapprochés les uns des autres dans l'espace. Au stade de la famille a succédé celui de la tribu. - Naturellement, n'ayant pas ici à faire même une esquisse de l'histoire de l'humanité. nous n'entrerons pas dans les débats qui se poursuivent aujourd'hui autour de ces appellations.

(.I) Voir Giraud-Teulon, Les origines du mariage et de la famille. Depuis la publication de ce livre, bien des recherches intéressantes ont été faites et publiées. Leurs résultats, consignés notamment dans les volumes successifs de L'Année sociologique, restent 'fragmentaires. Il n'a pu encore en être fait une synthèse digne d'être citée, ce qui tend à faire croire qu'elles n'ont qu'une portée assez limitée. 
Nous ne chercherons pas, notamment, si les groupements initiaux n'auraient pas mieux mérité les noms de clans, comme le proposait Letourneau, ou de hordes, comme le voudrait Durkheim. Nous dirons seulement que de la tribu sont sortis de nouveaux types sociaux, quand elle s'est fixée au sol et s'est réunie à d'autres tribus analogues, soit pacifiquement, soit plus souvent à la suite dé guerres. C'est ainsi que sont nées les cités grecques, les royaumes asiatiques, l'empire romain, et finalement les nations modernes. - Dans celles-ci, d'ailleurs, on retrouve les modes d'agrégation primitifs. D'une part, la famille y subsiste ; et même le principe de parenté s'est élargi, car sur lui repose maintenant la race. Cette dernière est comme une famille agrandie, dont tous les membres ne sont point parents, mais pourraient l'être: car ils se reconnaissent vaguement une origine commune et ils admettent la possibilité de s'unir entre eux ; c'est le groupe dans lequel existe, comme on 1'a dit, "la libre circulation du sang ". Une même race peut s'étendre sur diverses nations, et une même nation englober diverses races. D'autre part, le voisinage préside aux rapports habituels des membres d'une nation. Ceux-ci sont groupés dans notre pays en communes, cantons, arrondissements, départements ; ailleurs, en districts et en provinces. La façon dont s'effectue cette agglomération est suivie attentivement par les démographes ; ils ont montré l'importance qui s'attache, non seulement au chiffre total de la population d'un Etat, mais aussi à sa 
distribution entre les centres urbains et les campagnes, à sa densité et même à ce qu'on a appelé sa " densité morale ", c'est-à-dire à la facilité et à la rapidité des communications entre ses membres. En somme donc, les deux principes de rapprochement originaires, parenté et voisinage, se maintiennent à travers les siècles.

Mais, à côté d'eux, il s'en est établi d'autres. Ce fut le cas, d'abord, pour le principe professionnel. Dans toute société un peu étendue, s'opère une division du travail. Certains hommes se consacrent à une tâche particulière. De là naissent entre eux des liens de collaboration, puis de confraternité. L'analogie de leurs préoccupations les réunit. C'est ainsi que se sont formés, au moyen-âge, les corporations, de nos jours les syndicats. Il suffit de citer ces noms pour faire deviner l'importance de ce mode de groupement. On peut dire que c'est lui qui constitue les organes de la vie sociale moderne.

Avec les professions, il ne faut pas confondre les classes. La profession embrasse tous les hommes dont l'activité sert à produire un résultat donné, quel que soit le rang de ces hommes sur l'échelle sociale. Ainsi, la profession universitaire embrasse, en France, tous ceux qui donnent ou dirigent l'enseignement public, depuis les recteurs jusqu'aux instituteurs des moindres villages. Au contraire, la classe embrasse tous les hommes qui se trouvent à un même niveau sur l'échelle sociale, quel que soit leur genre spécial d'activité. Pour reprendre le précédent exemple, les recteurs 
feront partie de la classe la plus élevée, côte à côte avec les chefs, des grands services nationaux et avec les dirigeants du monde politique, intellectuel et économique; tandis que les instituteurs appartiendront à une classe modeste, où ils voisineront avec la plupart des employés des administrations publiques et avec certains travaili: leurs de l'industrie privée. Le groupement par' classes prend aujourd'hui une importance considérable, depuis que les classes les plus humblest ont appris à établir entre leurs membres une étroite solidarité pour formuler et défendre des revendications communes. La notion de classe sociale, qui a donné lieu à tant de controverses (I), arrive, par l'effet de la lutte des classes, à se préciser.

A côté de ces groupements, il.faut enfin en signaler d'autres, qui reposent sur la communauté de buts librement choisis. Ce sont les sociétés ou associations privées, nées dans les intérêts si variés que le jeu complexe de la vie moderne fait surgir. Dans l'ordre économique, on citera comme. telles les sociétés de commerce ; dans l'ordre intellectuel, les associations scientifiques, littéraires, artistiques ; dans l'ordre religieux, les congrégations; dans l'ordre politique, les partis. Le seul plaisir de se trouver réunis fait naître les salons, les cercles, les associations amicales. Celui de faire

(1) Voir notamment Cyr. Van Overbergh, La classe sociale, et Arthur Bauer, Les classes sociales. 
le bien en commun crée les sociétés philanthropiques. La coopération sous toutes ses formes s'organise et. s'étend. Elle multiplie ses œuvres et diversifie ses produits. Par elle, toutes les fins les plus nobles que l'homme peut concevoir se voient peu à peu atteintes, au moins partiellement. Sans doute, un tel mouvement porte aussi en lui-même ses faiblesses et ses dangers. Au total, cependant, il est généreux et utile. L'avenir, croyons-nous, lui appartiendra. 


\section{CHAPITRE XII}

LA VIE SOĊIALE.

Les éléments de la société étant déterminés, il faut les voir à l'œuvre. Quel est donc leur rôle,? et quelles fonctions remplissent-ils? C'est leur activité qui va produire les phénomènes sociaux, Nous devons dès lors chercher à énumérer ceux-: ci, ou plutôt les ordres divers auquels ils se rattachent. Puis nous examinerons les rapports de ces différents ordres entre eux.

Les faits sociaux sont si nombreux, si divers et si complexes, que leur classification constitue une tâche éminemment délicate et périlleuse. Les tentatives à cet égard ont été multiples, et au. début fort divergentes. Depuis quelques années, des rapprochements se sont produits, et il semble qu'on soit assez près d'arriver à l'accord. Les: grandes catégories de phénomènes sociaux peu-: vent être considérées comme dès maintenant éta- 
blies. Indiquons les principaux points de vue où l'on s'est placé pour les distinguer.

Un renommé sociologue belge, $M$. Guillaume De Greef, qui fut recteur de 1'Université Nouvelle de Bruxelles, a voulu appliquer à leur classification un principe tiré d'Auguste Comte (I). On sait que ce grand esprit, en établissant sa série hiérarchique des sciences, les disposait dans l'ordre de la complexité croissante et de la généralité décroissante de leurs objets. Il distinguait ainsi les phénomènes astronomiques, physiques, chimiques, biologiques, sociaux. M. De Greef a sousdistingué. Il a tenté, pour les phénomènes sociaux, ce que Comte avait fait pour l'ensemble des phénomènes naturels. Il a ainsi divisé les phénomènes sociaux en sept catégories: phénomènes économiques, génésiques (c'est-à-dire familiaux), artistiques, intellectuels (comprenant à la fois la religion et la science), moraux, juridiques, politiques. Cette classification, qui a obtenu un certain succès, témoigne à coup sûr d'une véritable ingéniosité et contient des données à retenir. On pourra toutefois regretter que l'auteur ait trop strictement voulu suivre Auguste Comte. C'est ainsi qu'il confond en un même ordre la religion et la science, parce qu'il croit celle-ci appelée à remplacer celle-là, sans voir que la religion peut

(r) Dans ses ouvrages intitulés : Introduction \& la sociologie (2 yolumes) ; Les lois sociologiques ; Le transformisme social, etc... 
conserver son domaine propre à côté et en dehors de celui des recherches proprement scientifiques. C'est ainsi encore qu'il insiste à l'excès sur le caractère de généralité décroissante des phénomènes compris dans ses catégories successives. L'on peut dire, sans doute, que l'organisation politique est la plus complexe de toutes, en ce sens qu'elle porte la marque de l'organisation économique, de l'organisation intellectuelle, de 1'organisation morale. Mais est-on autorisé par làmêmé à ajouter qu'elle est moins générale que toutes celles-ci ? $\mathrm{Y}$ a-t-il des groupes sociaux qui n'aient aucune organisation politique? Là où l'on croit le constater, n'est-ce pas parce que l'organisation domestique en tient lieu? Et, en ce cas, c'est elle qui devient l'organisation politique. De même, qui peut établir que les phénomènes moraux sont moins généraux que les phénomènes artistiques? On voit, par ces simples remarques, ce qu'il y a de conjectural dans cette intéressante : tentative.

La théorie de M. De Greef était inspirée par: Comte. Mais elle l'était aussi, dans une certaine mesure, par Herbert Spencer. L'école organiciste a suivi plus exclusivement les traces de ce. dernier. Elle estime qu'il. convient de distinguer, dans la vie sociale, les trois grandes fonctions que la biologie a relevées dans la vie individuelle: celles de nutrition, de reproduction et de relation.. La fonction de nutrition est représentée, ici, par les phénomènes économiques. Car ceux-ci ont tous pour but la richesst, qu'ils se réfèrent à sa 
production, à sa circulation, à sa répartition ou à sa consommation. Or la richesse sociale correspond bien à l'aliment organique, à prendre ces deux termes dans les acceptions larges que leur donnent les sociologues et les biologistes, puis- . que la richesse est tout ce qui sert à l'entretien de la vie. Quant à la fonction de reproduction, elle se traduit par ce que M. De Greef appelle les phënomènes génésiques, d'une' expression qui est peut-être trop strictement biologique, et à laquelle il vaudrait sans doute mieux substituer son synonyme proprement social : les phénomènes domestiques. Enfin la fonction de relation est en quelque sorte double. Parmi les faits sociaux qu'elle renferme; les uns n'impliquent pas et les autres impliquent 1'intervention de 1'Etat. Comme on le sait, l'Etat n'est pas toute la société. C'est seulement la société unifiée pàr un gouvernement et par des lois. En dehors de lui, demeure logiquement une grande partie des relations sociales. Ce sont les relations morales, intellectuelles; artistiques, religieuses. Celles-ci forment donc un groupe distinct des relations juridiques et politiques. Les dernières ont un caractère plus précis, mais aussi plus coercitif. C'est en elles surtout qu'on voit, disent les organicistes, s'affirmer l'unité de l'être social.

Dépouillons ces vues de ce que leur expression a de trop particulier, de ce qui met sur elles la marque exclusive d'une école, pour en dégager un fond d'idées qui puisse être accepté par tous. Pour cela, on peut renoncer d'abord à faire une 
classe à part pour les phénomènes de l'ordre domestique. Car, abstraction faite de leur base organique, laquelle relève de la physiologie, ils rentrent dans la catégorie des phénomènes moraux, dont on les considérera comme une simple section. Puis, on peut aussi renoncer à dire que les phénomènes économiques représęntènt la vie de nutrition; car cela n'ajoute pas grand chose à leur définition, et cela pourrait la rétrécir à l'excès. Ils relèvent d'ailleurs, à dire vrai, de la vie de relation, aussi bien que tous les autres faits sociaux. On aperçoit de la sorte que la division des phénomènes sociaux se simplifie grandement. D'un côté sont les faits de la vie matérielle, c'està-dire les phénomènes économiques. De l'autre côté sont les faits de la vie mentale. Certains de ceux-ci sont concevables sans 1'Etat: ce sont les phénomènes moraux, intellectuels, artistiques, religieux. Certains sont inconcevables sans lui, et ils en font la substance même : ce sont les phénomènes juridiques et politiques. Une semblable classification est éminemment simple. Elle n'emploie. que des termes connus et compris de tous. Elle utilise des distinctions que le sens commun. avait faites depuis longtemps, et qui étaient passées dans toutes les langues. N'est-ce pas une assez forte présomption en faveur de son exactitude?

Nous venons d'indiquer les principales catégories dans lesquelles se rangent les phénomenes sociaux. Il faut maintenant aborder la ques- 
tion des rapports que soutiennent entre elles ces diverses catégories.

D'ordinaire, on pose cette question comme il suit: quelle est celle de ces catégøries qui domine les autres? Et des réponses fort diverses lui sont alors données. Elles se rattachent généralement à l'un des deux grands courants opposés, que l'on pourrait appeler le courant matérialiste et le courant idéaliste. Suivant le premier, le fait social fondamental, c'est le fait économique. Suivant le 'second, 'c'est le fait intellectuel. Le type le plus connu des systèmes du premier groupe, c'est la theorie de Karl Marx. D'après celle-ci, les besoins primordiaux de la vie étant d'ordre économique, ce seraient les moyens de les satisfaire, et tout d'abord la constitution de 1'outillage productif, qui donneraient l'essor à tous les autres ordres de phénomènes sociaux : ils seraient la "base " dont ceux-ci formeraient la " super-structure ". Cette théorie a été inspirée à Marx par le spectacle des faits auxquels il avait assisté : la transformation de l'ancienne production à la main en production à la machine avait amené dans les deux pays où il vécut, l'Allemagne et l'Angleterre, de profondes mọdifications sociales des espèces les plus variées. C'est en généralisant cette constatation qu'il était arrivé à sa doctrine du matérialisme historique, ainsi appelée parce qu'elle croit tout le développement de l'histoire dominé par le facteur matériel .Nous aurons à peine besoin de faire ressortir ici ce qu'elle a d'étroit, d'incomplet, d'unilatéral. D'une part, la constitution de l'ou- 
tillage productif ne commande même pas l'ordre économique tout entier. M. De Greef, qui dans sa large synthèse a fait une certaine place aux idées de Marx, estime que, parmi les phénomènes économiques, c'est plutôt la circulation qui est le phénomène socialement primordial. Car c'est en passant de mains en mains que la richesse prend vraiment le caractère social. D'autre part, et sans contredit possible, l'ordre économique ne commande pas l'intégralité de la vie sociale. Il est clair, en effet, que le progrès des lettres, des beaux-arts, des sciences même, s'il se relie dans une certaine mesure au progrès économique, a pourtant ses causes propres qui sont fort distinctes de celui-ci. Pour n'en citer qu'un exemple, les systèmes philosophiques naissent les uns des autres, beaucoup plus que chacun d'eux ne naît des conditions économiques du milieu ambiant. La doctrine de Marx apparaît donc ici avec évidence comme inadéquate à la réalité.

Tout à fait contraire est la théorie d'Auguste Comte. On pourrait la prendre comme le type des systèmes du second groupe: nous voulons dire de ceux qui voient dans le fait intellectuel le phénomène social fondamental. Car Comte est, sur ce point, franchement et pleinement idéaliste. Pour lui, c'est la pensée, et avant tout le savoir abstrait, qui domine toute la constitution sociale. La conception que l'homme se fait de l'univers est, à ses yeux, ce qui caractérise le mieux chaque état social. On sait, en effet, qu'il divisait toute 1'histoire humaine en trois états: 1'état théologi- 
que, l'état métaphysiquẹe, l'état positif ; qu'il distinguait dans le premier les âges successifs du fétichisme, du polythéisme, du monothéisme, et que dans le troisième il entrevoyait, après l'âge actuel, caractérisé par le développement des savoirs particuliers, ou âge de la spécialité, un âge futur où florirait une philosophie synthétique et positive à la fois, l'âge de la généralité. A chacune de ces époques, c'est la science et la philosophie qui, d'après lui, ont inspiré l'art, la politique et la technique. - Nous reconnaissons bien volontięrs ce qu'il y a d'élevé et partiellement d'exact dans une semblable doctrine. Nous croyons toutefois qu'elle ne peut pas être poussée aussi loin que son auteur l'aurait voulu. Tout à l'heure nous revendiquions l'indépendance de la philosophie par rapport à 1'économique; ici nous admettrons 1'indépendance relative de l'àrt, de la politique et même de la technique par rapport à la science. Les initiatives du musicien, celles de l'homme d'Etat, d'ordinaire même celles de l'agriculteur ou de l'industriel, ne se puisent pas dans les laboratoires du physicien, encore moins dans le cabinet du penseur. Le facteur proprement intellectuel ne régit pas tout dans la société.

Que conclure de là ? C'est qu'il n'y a point un ordre de phénomènes sociaux auquel tous les autres se subordonnent. Mais cela fait-il qu'il n'y ait pas de liaison entre les divers ordres? En aucune manière. A notre avis, au contraire, tous sont très étroitement unis. Même, dans la plupart des cas, ils se pénètrent réciproquement. Pre- 
nons un exemple. Une guerre aujourd'hui est, essentiellement, un phénomène politique. Car elle résulte de l'inimitié de deux ou plusieurs nationalités, ou du moins de leurs gouvernements respectifs. Mais elle a, simultanément, une face économique: la concurrence de deux séries de producteurs sur le marché international. Elle a une face intellectuelle: le contraste de deux civilisations, de deux mentalités, de deux langues. Même des ordres de faits qui y semblent assez étrangers s'y voient mêlés : des deux côtés on fait appel à l'art, à la religion, à la morale, au droit, pour exalter l'ardeur des combattants. Ainsi toutes les catégories de phénomènes sociaux se trouvent représentées dans une semblable tragédie. Sans prendre un aussi vaste exemple, portons nos yeux sur un drame plus réduit: un procès en cour d'assises, si l'on veut. Celui-ci est d'ordre juridique par la procédure suivie, d'ordre moral par la question qui s'y pose. Les impulsions auxquelles les acteurs ont obéi sont souvent d'ordre économique. Des influencés d'ordre politique viennent. dit-on, parfois s'y exercer. Et, pour ne pas oublier les côtés accessoires, la science $y$ intervient avec les experts, la religion avec les aumôniers des prisons, l'art par la reproduction des scènes d'audience. C'est dire qu'il $\mathrm{v}$ a, à chaque instant, concours et interaction des divers ordres. Tantôt 1'un domine, et tantôt c'est un autre. Mais aucun n'est négligeable et, dans 1'ensemble. chacun a un rôle indispensable à jouer.

Seulement, ce qu'on peut se demander, c'est si 
l'analyse qui les sépare a une portée vraiment objective. Nous venons de raisonner comme si chacun de ces ordres possédait une existence séparée. C'est même parce que nous admettions leur séparation initiale que nous devions ensuite chercher à opérer un rapprochement partiel entre eux. Mais avions-nous raison de l'admettre? On en peut assęz sérieusement douter. Il se pourrait fort bien, en effet, que les catégories créées fussent sans réalité objective. En somme, les hommes en société agissent et leur action, suivant la face par laquelle on l'envisage, paraît surtout économique, ou surtout morale, ou surtout politique, etc... Au fond, pourtant, elle est toujours et uniquement sociale. Les distinctions que nous y intro duisons sont l'œuvre de notre esprit. Et c'est parce qu'il en reconnaît le caractère subjectif, donc un peu factice, qu'il est amené à les tempérer par l'établissement ultérieur de rapports entre les objets distingués. Ici, comme partout, la science a du fragmenter, pour le comprendre, ce qui dans la réalité était indivis. De l'un elle a fait du multiple, sauf à essayer ensuite d'en reconstituer l'unité (I).

(I) Entre les classifications des phénomènes sociaur indiquées dans cette étude, on n'a pas trouvé jusqu'ici celle qui est adoptée dans L'Année sociologique, et l'on va en savoir la raison. Voici les grandes divisions de ce livre : sociologie religieuse ; sociologie morale et juridique ; sociologie criminelle et statistique morale ; socio- 
logie économique ; morphologie sociale ; divers (langage, art, technique). Nous aurions beaucoup d'objections à faire a une semblable classification. Elle réunit la morale et le droit, et dans. le droit elle absorbe la politique. En revanche, elle dissocie le droit pénal et le droit civil. Les éléments réunis dans la rubrique " divers " sont vraiment trop hétérogènes. Mais surtout, on ne sait pas dans quel sens prendre ce tableau. La sociologie relig̀ieuse est placée en tête, sans doute parce que la religion a paru aux auteurs de L'Année, la forme primitive de toute culture. Seulement, c'est oublier que la pensée religieuse est en quelque sorte la fleur poussée sur une tige constituée par le solide réseau de la morale et du droit, et nourrie par une racine d'ordie économique. C'est aussi oublier que, avant les fonctions sociales, il faudrait envisager les structụres où elles se manifestent et dont l'étude constitue, suivant les expressions de L'Année, la morphologie sociale. En somme, cette classification a quelque peu l'apparence d'une pyramide rénversée. Nous estimons qu'i1 n'y a pas lieu de s'y arrêter longuement. Car elle peut n'être, dans la pensée de ses auteurs, qu'un ordre commode pour ranger les livres parus récemment et les matieres y contenues. Et à ce titre, l'arbitraire luị est permis, comme il le serait à l'ordre adopté pour disposer les volumes d'une biblióthèque. 


\section{CHAPITRE XIII \\ 1 \\ L'ÉVOLUTION SOCIALE.}

La société ne demeure point immobile. Ses structures se modifient sous 1'action de leur fonctionnement. De multiples et complexes problèmes se posent au sujet de cette évolution.

D'abord, quelle en est la cause? Il ne suffit pas de dire que le jeu des organes sociaux les transforme. Car le jeu d'une machine, par exemple, ne fait que l'user, et tout au contraire, dans la mécanique sociale, non seulement l'usure se répare, mais le mécanisme se perfectionne normalement par son usage même. D'où vient cette curieuse propriété?

Deux causes, croyons-nous, concourent à l'expliquer. L'une est la nature du moteur initial de l'évolution. L'autre est la nature du principal moyen employé.

Le moteur, c'est le désir du mieux. L'homme, incessamment, aspire à une amélioration dè sa condition. Il ne tend pas seulement, comme tout 
être, suivant la définition de Spinoza, à persévérer dans son être. Il aspire aussi 'à l'agrandir, à l'élever. Telle est, à nos yeux, la caractéristique de l'espèce humaine entre toutes les espèces vivantes.

Quant au moyen, c'est le développement de l'intelligence. Comme tout autre organisme, l'homme cherche à s'adapter à son milieu. Mais cet effort pour l'adaptation prend chez lui un aspect tout particulier. Il revêt très vite et très généralement la forme mentale. Chez l'animal, il est impulsif et irraisonné. Chez 1'homme, il est réfléchi. La volonté, ici, est guidée par le jugement. Aussi l'effort acquiert-il une tout autre ampleur et une tout autre sûreté. Une extension presque illimitée devient possible pour lui. L'individu ne subit plus simplement, comme dans l'animálité, 1'action de son milieu. Il réagit sur lui à chaque instant, et, s'il est modifié par lui, il parvient à le modifier à son tour.

L'avantage considérable que présentent les créations de l'intelligence, c'est qu'elles ont la propriété de pouvoir s'additionner, s'accumuler. Ainsi s'explique que, au cours des siècles, les découvertes se continuent et s'engendrent l'une l'autre. Le total des idées qui guident 1'humanité, des pratiques qu'elle suit, des objets qu'elle utilise, va de la sorte en s'amplifiant sans cesse. Gabriel Tarde, en étudiant la nature du capital (I),

(1) Dans sa Psychologie économique, 
a dit qu'il n'est autre chose que l'ensemble de nos inventions șuccessives. Pour que cette ingénieuse définition soit exacte, il faut seulement ajouter qu'il s'agit là d'inventions concrétisées dans des produits ouvragés; ou tout au moins il faut distinguer un capital mental et un capital matériel, 'en appliquant au second cette restriction. Ainsi, notre espèce, en principe, voit s'accroître d'âge en âge le trésor de son savoir théorique, de son savoir pratique et de ses moyens de subsistance et d'activité.

Toutefois, l'on sait bien qu'il n'y a rien d'absolu dans ce principe. La règle que nous venons de poser né souffre que trop d'exceptions. S'il $y$ a le plus ordinairement marche en avant, il $y$ a aussi, fort souvent, des arrêts et même des reculs. D'où cela vient-il? Parfois, d'une sorte de torpeur qui envahit les esprit et entrave l'essor de l'intelligence. Plus souvent peut-être, de l'action destructive d'un groupe moins avancé sur un autre qui l'est davantage. L'immobilité de la civilisation chinoise pendant des siècles paraît dûe à une cause du premier ordre. La ruine de la civilisation romaine au $\mathrm{v}^{\theta}$ siècle de notre ère tient à une cause du second. - Existe-t-il, maintenant, une loi de ces reculs? On a plusieurs fois tenté d'en formuler une, mais non, semble-t-il , avec un entier succès. On a parlé, Taine notamment, de la réversion des formes sociales. L'édifice politique se détruirait dans l'ordre inverse de celui qui a présidé à sa création. Des strates superposés qui le forment, les plus récents s'écrouleraient 
d'abord, et les plus anciens résisteraient davantage. L'idée est juste d'ordinaire, car ce sont les premières acquisitions de la culture qui, étant les plus fondamentales, les plus indispensables, risquent le moins d'être anéanties. Elle ne saurait pourtant être prise à la lettre. La régiression ne. ramène pas toujours des formes disparues. Ainsi, sur les ruines de l'empire romain,. n'ont pas, reparu de petites cités autonomes comme celles qui existaient en Grèce et en Italie au vir $^{\ominus}$ siècle avant notre ère, mais se sont formés de vastes royaumes, ébauches des grandes nations modernes. Tarde avait même cru pouvoir établir, à ce sujet, une règle contraire à celle de la réversion. Il l'appelait le principe d'irréversibilité. Jamais, disait-il, une société ne repasse par un stade antérieur. Dans ses reculs il lui reste, de son évolution, quelque chose qui différencie l'état nouveau de l'état originaire. Nous le voulons bien, mais à condition qu'on admette qu'il s'y trouve aussi certaines choses, et même d'ordinaire en plus grand nombre, qui le rappellent. En somme, tout en ces matières est question d'espèce, et i1 serait téméraire de poser une formule trop générale. Signalons seulement un dernier cas, assez curieux, de réversion: celui du retour intentionnel au passé. Des sociologues polonais, assez peu connus en France, Paul Weisengrün, Casimir de Kelles-Krauz, l'ont indiqué sou's le nom de: rétrospection révolutionnaire (I). En cas de révolution,

(I) V. Casimir de Kelles-Krauz, La loi de retrospection 
un pays reprend volontiers des institutions poli. tiques d'autrefois. La Révolution française a cru rétablir certaines magistratures de la République romaine : c'est ce qu'indiquaient notamment, dans ses constitutions, - les termes de consulat, de tribunat, de sénat. La Restauration a voulu faire renaître l'ancien régime. La seconde République a donné à ses assemblées les noms de celles de la première. Le second Empire a cherché à copier . le premier. Présentée ainsi, cețte vue n'aurait qu'un intérêt limité. Mais elle peut être généralisée et alors on trouve derrière elle une conception d'ensemble de l'évolution.

Cette conception, c'est celle qui voit dans l'histoire humaine un retour périodique à des formes antérieures disparues. Elle a pris chez les sociologues divers aspects qu'il importe de noter. Auguste Comte remarquait (après son maître Henri de Saint-Simon) qu'il se produit une alternance entre deux sortes de périodes: les unes qu'il appelle organiques, les autres qu'il nomme critiques. Dans les premières, un type social s'élabore; dans les secondes, il se détruit. Le moyenâge a été une période organique. De la Renaissance à la Révolution française se prolonge une période critique. De nos jours se crée une organisation nouvelle. - Herbert Spencer, pareillement, a posé une loi, qu'il appelle la loi du

révolutionnaire, travail paru dans le tome II des Annales de l'Institut international de sociologie, 1896. 
rythme, et qui dépasse le monde social pour régir l'univers entier. En vertu de ce principe, il y aurait tour à - tour évolution et dissolution, ou encore intégration et désintégration, c'est-à-dire concentration de la matière avec dissipation du mouvement, puis absorption du mouvement avec diffusian de la matière. Sous d'autres expressions, et avec le souci de choisir des termes qui relient la mécanique sociale à la mécanique physique, c'est à peu de choses près la théorie d'Auguste Comte.

Dans l'une comme dans l'autre, les phases de l'histoire forment en quelque sorte des couples, des dyades. Pour un grand penseur différent, Hégel, elles forment des triades. Une thèse, une antithèse et une synthèse se succèdent incessamment. C'est la loi de l'histoire, c'était déjà la loi de la nature, c'est la loi de la logique étèrnelle. Dans la société, cette alternance prend l'aspect que voici. Un régime s'institue : c'est la thèse. 'Mais il suscite des mécontentements qui finissent. par le renverser; les opposants dominent à leur tour: c'est l'antithèse. Les partisans de l'ancien état de choses reprennent courage, obtiennent oertaines satisfactions; i1 s'établit un régime mixté : c'est la synthèse. A son tour, celle-ci servira de point de départ pour une nouvelle évolution ternaire. On pourrait caractériser ces trois étapes, dans le domaine social, en les appelant: l'action, la réaction, la transaction. Et l'on en trouverait un assez bon exemple dans ce qui s'est passé en France, il y a un siècle environ, avec le 
premier empire, la restauration, la monarchie de juillet.

Comme on le voit, dans le système de Hegel, la synthèse marque un progrès sur les deux stades 'antérieurs. C'était d'ailleurs aussi l'idée d'Auguste Comte, pour lequel chaque nouvelle période organique bénéficie, par rapport à la précédente, des apports de la période critique intermédiaire. C'était également l'idée d'Herbert Spencer. Une expression intéressante de ce qu'il y a de commun à ces systèmes a été proposée par un sociologue français, assez récemment disparu, Raoul de la Grasserie, dans son étude intitulée: De la forme graphique de l'évolution. Il a tenu compte de l'idée émise autrefois par Vico, 1'idée des ricorsi ou retours cyćliques de la nature sur elle-même; mais il l'a combinée avec l'idée du progrès. Suivant lui, l'évolution ne se fait pas suivant une ligne droite, comme le vulgaire l'admet volontiers. Elle n'affecte pas non plus la forme d'un cercle, comme le voulait Vico dans sa conception un peu trop simpliste. Elle prend plutôt 1'allure d'une spirale (voir le graphique de la page suivante). A chaque tour de spire, l'humanité arrive à un point nouveau, A', A', placé sur la même ligne verticale que le point initial $A$, mais sur une ligne horizontale plus haute. Dans l'intervalle s'est effectué le jeu des thèses et des antithèses, comme celui des organisations et des critiques, des intégrations et des désagrégations. Le résultat est une synthèse qui, malgré les oscillations et les reculs partiels, marque une certaine progression. 
Toutes les théories que nous venons d'indiquer nous paraissent avoir une portée sérieuse. Chacune d'elles peut s'autoriser d'exemples historiques. Chacune renferme donc une certaine

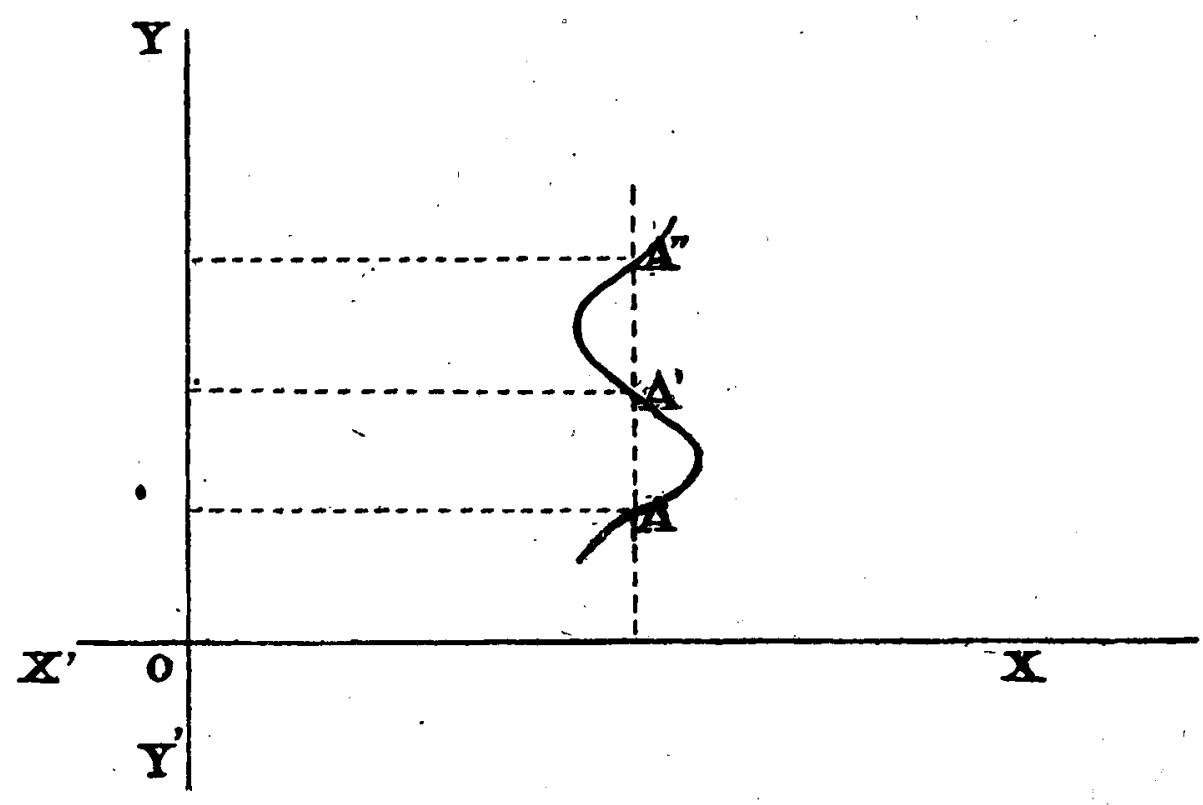

part de vérité. Mais aucune, à notre avis, ne saurait être donnée comme exprimant la vérité intégrale. L'évolution humaine est chose trop complexe et trop diverse pour tenir dans une formule simple. Elle ne s'est pas partout et toujours accomplie suivant les mêmes règles. Il faut distinguer ici entre les temps, entre les lieux, entre les institutions. Suivant l'époque qu'on envisage, suivant le pays étudié, suivant l'ordre des faits dont on 
suit le développement, on voit apparaître des aspects nouveaux de l'histoire, qui ne relèvent point de règles connues. Cela ne veut pas dire que les formules relatées plus haut soient inutiles. Au contraire, plus on avance dans les études sociales, plus on a l'occasion de constater des cas auxquels certaines d'entre elles s'appliquent d'une façon frappante. Mais tantôt c'est l'une et tantôt c'est l'autre, ce qui prouve que nulle ne suffit à tout expliquer, voire même à tout résumer. Et à côté d'elles toutes, il faut tenir compte de la multitude des cas qui ne rentrent dans aucune, il faut réserver les, droits de l'incompris et de l'inconnu....

En tout cela, un point paraît se dégager avec certitude : c'est qu'évolution ne signifie pas nécessairement progrès. Evolution est un terme neutre qui, n'implique aucun jugement de valeur sur la qualité du processus constaté. Progrès comporte au contraire une qualification louangeuse. Or, il faut bien reconnaître que, dans une foule de cas, cette qualification ne peut être donnée aux évolutions accomplies. Non seulement les progrès sont toujours coupés de temps d'arrêt et de régressions; mais on doit ajouter qu'ils ne sont jamais que-locaux et partiels. Ce qui fait le progrès d'un peuple - une victoire par exemple, fût-ce une victoire diplomatique ou industrielle - fait le recul de l'autre. Et, chez une même nation, le progrès dans une sphère peut s'accompagner d'un recul dans un autre domaine. Ainsi le progrès dans l'organisation militaire peut en- 
traîner une régression vers les mœurs belliqueuses et la barbarie atavique. Les diverses formes de progrès ne sont pas nécessairement solidaires les unes des autres. C'est même une question de savoir si celle qui sans doute importerait le plus, à savoir le progrès moral, est liée étroitement au progrès intellectuel et au progrès matériel. Nous croyons, pour notre part, que le développement. de la culture est en principe favorable au perfectionnement éthique. Mais nous ne pouvons méconnaître qu'il y a, à cette règle, de nombreuses et graves exceptions.

Le mot de progrès soulève, d'ailleurs, un dernier problème. Tout jugement de valeur est nécessairement subjectif. La qualification de progrès l'est par là-même. Nous appelons progrès la marche de la société vers notre idéal. Mais cet idéal est tout personnel, et qui nous garantit qu'il soit fondé ? On est porté à en douter, quand on constate les oppositions qu'il rencontre. L'idéal varie en effet avec les individus. Aussi jugent-ils différemment presque toute chose. Ce qui parait progrès à l'un semble donc recul à l'autre. En matière politique, par exemple, un parti juge excellentes et progressives des mesures que les autres estiment néfastes et régressives. - Comment échapper à cette difficulté? -Son examen complet sortirait évídemment des cadres de la présente étude. Ce qu'on peut dire toutefois, c'est qu'il est logique d'adopter comme critères du progrès les principes généralement reçus dans les nations: qui tiennent la tête du mouvement humain. On 
considérera donc légitimement comme progressives les évolutions qui assurent à la grande masse de notre espèce plus de satisfactions matérielles, intellectuelles et morales; pour régressives, celles qui diminuent ces biens si ordinairement souhaités. Au cas où ces critères eux-mêmes paraîtraient incertains ou contestables, une seule règle pourrait être recommandée au sociologue: celle qui consisterait, pour lui, à s'abstenir de toute qualification des événements constatés. Il se bornerait à décrire, il ne jugerait jamais. Ce serait une attitude plus strictement scientifique. Il est douteux toutefois que l'on puisse toujours l'observer. Le sociologue qui s'y tiendrait, devrait rigoureusement éviter de prononcer jamais le nom de progrès. Le terme d'évolution lui suffirait. , 


\section{CHAPITRE XIV}

\section{LA MÉTHODE SOCIOLOGIQUE.}

Dans les pages qui précèdent, nous avons indiqué les questions dont s'occupe la sociologie. Evidemment, elle n'est pas encore parvenue à les résoudre. Elle date de trop peu de temps pour cela. Mais enfin, elle tend avec activité vers ce but. Comment donc le fait-elle? Quelle voie peutelle suivre? En un mot, quelle est sa méthode?

Pour l'expliquer, nous distinguerons trois choses que trop souvent l'on confond. Ou plutôt, nous rappellerons d'un seul mot la distinction posée, au début de cette étude, entre trois séries de disciplines: les sciences sociales particulières, qui examinent les aspects spéciaux de la réalité; la sociologie, qui en dégage les principes les plus généraux ; les arts sociaux, qui s'occupent d'améliorer l'état de choses existant. Chacune de ces trois séries a sa méthode, déterminée par sa nature propre. 
I. - Voyons ce qui concerne, tout d'abord, les sciences sociales particulières. Sous réserve d'une liste plus complète qui en sera dressée ultérieurement, citons parmi elles, à titre d'exemples, la démographie, l'ethnographie, la science économique, l'histoire ou science des mœurs, l'histoire ou science des religions.

Ce qui caractérise leur méthode à toutes, c'est qu'elle est essentiellement inductive. Elle part de. la constatation des faits pour s'élever à la découverte des lois. Ses étapes successives sont les suivantes.

En premier lieu, vient l'obervation proprement dite. Quand il s'agit de faits qui se passent sous ses yeux, l'homme de science peut directement les constater. L'économie politique et sociale a décrit, à cet effet, différents procédés fort utiles. C'est d'abord le procédé statistique, qui donne à l'observation la précision des chiffres. C'est ensuite le procédé monographique, qui a des avantages et des inconvénients opposés à ceux du précédent: il fouille un point, au lieu de relever une surface; il pénètre plus avant, mais il manque de * généralité ; les esquisses qu'il permet de faire ont plus de vie, mais moins de portée. C'est enfin le procédé dit de l'enquête, qui associe dans une certaine mesure la monographie et la statistique, tâchant de bénéficier ainsi des prérogatives de l'une et de l'autre.

Mais quand il s'agit de faits qui ne se passent point sous ses yeux, 'l'homme de science n'a aucun de ces moyens à sa disposition. Il ne peut plus WORMS 
faire par lui-même ses constatations. Il est obligé de les émprunter à autrui, sauf à vérifier le témoignage ainsi porté. C'est ce qui a lieu dans deux cas: pour les phénomènes sociaux qui sont éloignés dans l'espace, et dont le contrôle direct est à la rigueur possible, et surtout pour ceux qui sont dépassés dans le temps, sur lesquels ce contrôle est devenu impossible. Dans l'un et l'autre cas, on tâche de recueillir des documents aussi précis qu'il se peut. Les uns sont des documents humains: les dires des voyageurs, dans le premier cas, ou ceux des hommes d'autrefois, dans le second. Les autres sont des documents non humains; ce sont les traces que les sociétés laissent d'elles-mêmes: monuments, habitations, vêtements, outils, armes, parures, etc... Ces documents matériels sont souvent plus utiles que les dires des hommes, parce qu'ils ne risquent pas d'être mensongers, au moins quand ils n'ont pas été dressés pour nous. Les uns et les autres doivent d'ailleurs faire l'objet d'une critique des plus attentives, et leur interprétation ultérieure est encore entourée de diffficultés de toutes sortes. L'ensemble de ces procédés d'utilișation des témoignages constitue ce qu'on pourrait appeler 1'observation indirecte.

A côté de l'observation directe et de l'observation indirecte, on cite parfois 1'expérimentation. Mais nous craignons que ce ne soit par suite d'une confusion. L'expérimentation est fort utile aux arts sociaux, comme nous le verrons tout à 1'heure. Dans les sciences sociales, au contraire, 
elle ne nous paraît pas de mise. Expérimenter, en effet, c'est produire soi-même un fait pour le constater avec précision. Or, en matière sociale, ce serait un procédé peu admissible. L'organisation sociale est chose trop grave, trop précieuse, pour qu'on veuille la changer uniquement afin de voir ce qui en résultera. On peut faire un tel changement en vue d'une amélioration à réaliser. On ne doit pas le faire dans un but de simple curiosité. Voilà pourquoi, alors que l'art social peut recommander l'expérimentation, la science sociale ne le doit pas (I).

Les faits une fois réunis par l'observation, il s'agit de lés grouper. Pour cela interviennent de nouveaux procédés : la classification, la recherche des causes, l'induction ou établissement des lois.

Sur la classification, nous ne nous étendrons pas longuement. Car ses procédés sont, en matière sociale, ce qu'ils sont en toute autre matière. Il s'agit, ici comme là, de rapprocher les êtres ou les faits qui présentent entre eux plus de similitudes que de différences, et qui en même temps offrent plus de ressemblances entre eux qu'avec tout autre être ou fait. Et partout il convient de peser les caractères plutôt que de les compter, c'est-â-dire de noter surtout ceux qui ont une im-

(i) Ajoutons d'ailleurs qu'elle risquerait de la conseiller en vain, l'homme de science ne $x$ disposant pas d'ordinaire de la force publique, qui permet les expériences les plus fructueuses. 
portance primordiale et qu'on peut nommer les caractères dominateurs, parce qu'ils apparaissent comme la cause des autres.

Nous sommes ainsi amené à nous poser le problème de la causalité. Ce problème ne revêt 'pas, dans les sciences sociales, le même aspect que dans les sciences physiques. I1 aurait plutôt le: même aspect que dans les sciences organiques. Expliquons-nous. Dans la physique proprement dite, un phénomène est appelé la cause d'un autre, lorsque d'une manière invariable il se produit avant lui et l'entraîne à sa suite. Par exemple, l'échauffement de l'eau est la cause de son ébullition. Mais, déjà dans le monde vivant, il n'en est plus de même. Ici la cause et l'effet apparaissent souvent d'une manière concomitante. Ou bien encore, un phénomène qui en entraînait un autre après lui subit ultérieurement l'influence de celuici, de telle sorte que ce qui était cause devient effet et réciproquement. Des exemples se trouvent dans les rapports de chaque organe avec sa fonction. C'est la structure d'un muscle qui explique son jeu, mais c'est aussi ce jeu qui rend raison de cette structure. Et, si une malformation dans, la structuure entraîne une entrave dans le jeu, réciproquement un obstacle qui paralyserait le jeu risquerait d'altérer la structure. D'autres' exemples se trouveraient encore dans les rapports des divers organes entre eux, des diverses fonctions entre elles. Un organisme est un tout harmonieux, dans lequel chaque partie a son influence sur chacune des autres. Ainsi son système 
nerveux et son appareil digestif sont en corrélation incessante; tout ce qui trouble l'innervation trouble par contre-coup la digestion, et réciproquement. - Il en est de même dans le monde social, à ceci près que, les structures et les fonctions étant ici plus complexes que dans le monde organique, les actions et réactions entre elles sont plus complexes également. Dans une société, la vie politique, la vie morale, la vie intellectuelle; la vie politique se pénètrent sans cesse, et leurs organes, c'est-à-dire les corps qui en assurent l'exercice, opèrent les uns sur les autres une pression ininterrompue. Comme l'on sait, c'est surtout pour servir des intérêts économiques que les chemins de fer ont été construits et développés ; mais ils ont aidé à la diffusion des idées et à l'établissement d'un régime démocratique; et ces phénomènes, à leur tour, ont fait évoluer l'organisation économique. Voilà un exemple tiré de la cinématique sociale. Il montre la transformation des causes en effets, à travers la succession des phénomènes. Mais considérons maintenant les choses du point de vue de la statique sociale. A un moment et en un lieu donnés, par exemple aujourd'hui et en France, coexistent ces trois choses: un réseau complet de voies ferrées, un intense mouvement des idées, un régime démocratique, ef chacune d'entre elles est, dans une certaine mesure, la cause des deux autres. Par là, on peut saisir le caractère de réciprocité que présentent les causes et les effets à une même date. - On vait ainsi combien la notion de cause est WORYS 
différente, dans le monde social et dans le monde organique, de ce qu'elle est dans le monde phy sique. Certains esprits ont été tellement frappés. de cette différence, qu'ils ont cru illégitime de désigner par un même mot deux choses si distinctes, et qu'ils ont proposé d'éliminer le terme de cause du domaine social. On l'y remplacerait par le terme de fonction. On ne dirait plus que deux ordres de phénoménes sociaux ont des rapports de causalité, mais simplement qu'ils' varient en fonction l'un de l'autre. - Nous ne croyons pas nécessaire, pour notre part; d'aller aussi loin, d'introduire un tel bouleversement dans les dénominations reçues. Nous dirons simplement qu'il y a, dans le monde social comme dans le monde organique, deux types différents de relations à envisager. dans chacun desquels peut se trouver la causalité. Les premières sont des relations de coexistence, les secondes sont des relations de succession. Dans les premieres, un des phénomènés en rapport peut sembler dominateur; mais le plus souvent les phénomènes subordonnés ont sur lui des influences réciproques qui font de lui, à son tour, leur tributaire partiel. Dans les secondes, le phénomène antécédent peut être dit la cause du phénomène conséquent ; mais d'ordinaire ce dernier prend bientôt sa revanche, si 1'on peut ainsi parler: il réagit sur son antécédent et il en devient, partiellement tout au moins, la cause ultérieure. - C'est assez dire combien est délicat, dans le monde social, ce problème de la recherche des causes . On comprend notamment qu'il faille s'y garder des affir- 
mations étroites, unilatérales. L'hormme d'étude -peut rarement se flatter d'y avoir découvert la cause d'un phénomène. Cela tient d'abord à ce que, dans ce monde complexe, de multiples causes concourent presque toujours à un même effet. Cela'tient aussi à ce que, comme nous venons de le montrer, une cause et son effet intervertissent fréquemment leurs caractères. - De ce qui préoède, découle enfin une règle pratique, que voici. On connaît les quatre méthodes pour la découverte des causes, distinguées par John Stuart Mill et dénommées par lui respectivement: méthode de concordance, méthode de différence, méthode, des variations concomitantes, méthode des résidus. Eh bien, les deux premières et la quatrième sont d'un emploi assez peu fructueux en matiere sociale. Car rarement on aura pu isoler des faits d'une manière assez complète pour réaliser des accòrds ou des contrastes parfaits entre eux. Mais la troisième peut rendre de précieux services. C'est elle en effet qui montre des séries de phénomènes variant en fonction l'une de l'autre. La statistique, notamment, en permet l'emploi. Par exemple, on a pu montrer, en France, le parallelisme des deux séries que forment dans le temps et l'espace la réduction de la natalité et l'accroissement de l'épargne, et on a été amené à penser que le second de ces faits peut avoir exercé sur le premier une influence décisive. Il v a même là un résultat qui paraît nettement acquis aux démographes (I). L'un au moins des procédés décrits

(I) J.-V. Talqvist, Recherches statistiques sur la ten- 
par Mill dans sa Logique reste donc, socialement parlant, de première importance.

Quand des rapports de causalité ont été découverts, il faut les généraliser. C'est l'opération qui porte le nom d'induction. On sait la prudenceavec laquelle elle doit être faite, en quelque matière que ce soit. Mais c'est en matière sociale que cette précaution s'impose tout particulièrement, et voici pourquoi. Logiquement, un rapport une fois établi avec certitude doit être vrai d'une vérité générale. Une cause produira toujours son effet, une connexion de phénomènes subsistera toujours. Mais, en fait, des causes qui n'agissaient pas dans le cas primitivement étudié peuvent intervenir dans le cas nouveau, des phénomènes qu'on. n'avait point remarqués dans le premier peuvent apparaître dans le second. Les prévisions se trouvent dès lors bouleversées. Ce n'est pas que 1'effet attendu ne se produise aucunement. Seulement, il est masqué par les effets plus apparents des facteurs qui viennent cette fois d'entrer en jeu. Tout cela est dû à la complexité et à la variabilité du monde social. Pour celui-ci donc, des lois ne peuvent être posées par induction qu'avec une extrême circonspection. Et, quand on les formu-

dance à une moindre fécondité des mariages, 1886. Arsène Dumont, Dépopulation et civilisation, i89o. D $\mathrm{D}^{\mathrm{r}} \mathrm{Jac}-$ ques Bertillon, La dépopulation en France, 19ri. Paul Leroy-Beaulieu, La question de la population, $2^{\circ}$ édition, 1913. René Worms, Natalité et régime successóral, 19i7. 
lera, il faudra se garder de leur attribuer une valeur universelle. Cette épithète serait excessive. On pourra simplement dire qu'elles ont une portée générale. Avec cette qualification plus modeste, on se garde contre les démentis de l'expérience; on réserve, comme il le faut toujours, l'avenir.

Parmi les lois sociales, il y a lieu naturellement de reconnaître deux catégories: les lois de coexistence et les lois de succession. Cela va de soi, puisque les lois sont des rapports de causalité généralisés, et que ces rapports comportent la dite division. Mais nous devons ajouter ici un mot en ce qui concerne les lois de la première sorte. La façon scientifique dont on considère aujourd'hui l'histoire a permis de ranger les organisations sociales dons un certain nombre de types "généraux. Tels sont le type de la cité dans l'antiquité gréco-latine, le type de la ville médiévale, le type de l'Etat occidental contemporain. Tels sont encore les types de la tribu arabe et de la tribu peau-rouge. Qu'est-ce qui caractérise chacun de ces types? La coexistence d'un certain nombre de traits, les uns tenant à l'organisation économique, les autres à l'organisation domestique, d'autres encore aux organisations morale, religieuse, intellectuelle, politique, etc... Ces traits sont liés les uns aux autres, ils se supposent et s'entraînent réciproquement, on les retrouve tous quand on découvre l'un d'eux. Le type résulte justement de leur liaison. Par conséquent, il consiste en une loi de coexistence. Ainsi, il n'est pas 
besoin de faire, de la découverte des types, l'objet d'une opération particulière des sciences sociales. Elle rentre dans les procédés que nous avons précédemment énumérés. Elle est une forme de l'induction.

Mais, avec l'induction, nous n'avons pas tout dit. Il reste un dernier procédé à signaler: la déduction. Il est vrai que celle-ci s'est vu parfois contester une place dans les sciences sociales. La raison nous en paraît être la suivante. Une des disciplines sociales qui s'étaient le plus vite et le plus complètement constituées, l'économie politique, fit souvent un emploi presque exclusif $\mathrm{du}$ procédé déductif. L'abus en devint palpable chez des écrivains comme Ricardo et Karl Marx. Une réaction était inévitable. Elle se fit sentir, sous la forme d'une protestation contre l'emploi de la méthode déductive, et d'un appel à l'induction. Les économistes de l'école historique ont mené cette campagne avec une grande vigueur et ils ont partiellement triomphé. Une sorte de délimitation est assez ordinairement admise aujourd'hui entre les domaines des deux méthodes. On laisse la déduction à l'art social, où nous verrons bientôt qu'elle joue nécessairement un rôle fondamental ; et on déclare que la science est le champ propre de l'induction. - Mais cela n'est pas, à notre avis, tout à fait exact. Sans doute, dans les sciences sociales, les procédés qu'on englobe sous le nom de méthode inductive (observation, classification, découverte des causes, induction proprement dite) sont les principaux. Toutefois, 
l'induction une fois faite, on peut légitimement asseoir sur elle la déduction. Quand un principe général a été solidement posé par une induction valable, on est en droit d'en tirer déductivement les conséquences, et de faire état de celles-ci dans la science. - Ce n'est pas tout. Dans le corps même de la recherche inductive, certaines déductions s'interposent. Car la déduction, c'est au fond le mode de raisonnement le plus usuel, celui qui de propositions établies fait sortir leurs suites logiques. Or, un semblable raisonnement, on s'en sert à toutes les minutes, et qui voudrait s'interdire d'y avoir recours? Eliminer la déduction, c'est mutiler l'esprit. A vrai dire même, c'est le frapper d'impuissance. On 'peut très correctement demander que la science n'emploie point la déduction sans la contrôler par la vérification directe des faits. On ne saurait sans absurdité proposer qu'elle n'y fasse jamais appel.

II. - Toutes les explications qui précedent, concernent la méthode des sciences sociales par;ticulières. Mais, pour nous, la sociologie générale ne se confond pas avec ces sciences. Elle n'en est pas la juxtaposition, elle en est la synthèse. Elle tire d'elle ses éléments, mais avec ceux-ci elle fait son œuvre propre. Il était donc nécessaire de montrer comment ces sciences procèdent, puisqu'ainsi seulement on peut se rendre compte de la façon dont la sociologie reçoit ses matériaux. Mais il reste à dire comment elle édifie sa construction. 
C'est, nous semble-t-il, par rapprochement qu'elle opère. Elle examine les résultats les plus généraux, acquis aux diverses sciences sociales: Puis elle les compare entre eux, les complète les uns par les autres, et les fusionne. Sòn rôle, en effet, nous l'avons vu, est de juxtaposer les diverses faces de la réalité sociale, dont chacune est du ressort d'une science sociale particulière. Elle tire aussi parti, pour sa construction, des données de la psychologie, et de celles des deux. autres sciences générales constituées avant elle, nous voulons dire la biologie et la cosmologie. Elle tâche ainsi de découvrir les lois d'ensemble? du monde social, en les rattachant même, quand cela est possible, à celles du monde vivant et à celles du monde physique. Les indications que nous donnons ici peuvent paraître un peu succinctes. On les verra se préciser dans un instant, quand nous aurons énoncé, au chapitre suivant:: les principales lois que la sociologie a dès à présent formulées. Bornons-nous à dire, pour le moment, que son procédé essentiel est la coordinar. tion.

III. - I1 reste enfin à nous expliquer, en peu de mots, sur la méthode des arts sociaux. Ceux-ci, à nos yeux, sont les disciplines tendant à organiser, à améliorer l'action sociale. Voici comment ces arts procèdent. Chacun d'eux pose d'abord un idéal supérieur qui doit dominer toute action. L'art économique, par exemple, prend pour idéal la diffusion du bien-être; 1'art moral, le règne du 
bien, ou celui du devoir. Puis cet idéal, l'art le confronte avec la réalité; pour voir le précepte qu'il doit formuler. Il fait ainsi ce qu'on a appelé "le syllogisme de l'action ". Etant donné l'idéal (majeure), étant donnés les obstacles qu'il trouve dans le réel (mineure), voici la règle à suivre pour s'y conformer autant que possible (conclusion). On voit qu'ici l'art opère par déduction. En somme, tandis que la science tend à s'élever du particulier au général, l'art descend du général au particulier.

Mais les conclusions de ses syllogismes, il les soumet à l'épreuve des faits. Si la pratique leur donne tort, il les abandonne, ou plutôt il les corrige en conséquence. Il emploie donc, au dernier stade de son processus, l'expérimentation. Celleci a été fort préconisée pour l'art politique (r), et elle n'est pas moins utile à l'art économique et à l'art moral. Nous ne pouvons insister ici sur la méthode des arts sociaux, qui est en dehors de notre sujet. Mais nous avons tenu à indiquer pour-

(1) Dans 'son livre sur La politique expérimentale, Léon Donnat a montré comment le législateur peut s'éclairer sur la valeur des textes qu'il adopte, en ne les appliquant d'abord que pour une certaine période (législation temporaire), ou pour une certaine fraction du territoire (législation séparée), ou bien encore en remettant leur application aux pouvoirs locaux (législation facultative), ce qui lui permet ensuite de les modifier, compléter ou abroger en plus complète connaissance de cause. 
tant d'un mot comment l'expérimentation et la déduction y jouent un rôle, beaucoup plus grand que celui qui leur revient dans la science (I).

(I) Pour le développement des principes posés en ce chapitre, on pourra consulter notre volume-intitulé Méthode des sciences sociales, qui forme le tome second. de notre Philosophie des sciences sociales. 


\section{CHAPITRE XV}

\section{LES LOIS SOÇIOLOGIQUES.}

La sociologie, étant la synthèse des sciences sociales; doit tendre à découvrir les lois les plus hautes du monde social. En a-t-elle déjà trouvé et établi de semblables? Il ne faut certes pas se montrer trop exigeant vis-à-vis d'elle, puisqu'elle n'est qu'à ses débuts. On peut pourtant lui demander légitimement de formuler déjà plus que dès promesses. Essayons donc de dresser l'inventaire sommaire de ses apports.

Trois sortes de lois sociales peuvent être distinguées, en se plaçant aux trois points de vue précédemment dégagés ( $\mathrm{I}$ ) et que nous appellerons ici, pour simplifier, les points de vue statique, dynamique et cinématique.

La statique établit les lois de coexistence. Une loi de ce genre caractérise un type social. Nous

(1) Dans notre chapitre $\mathbf{X}$. 
nous sommes un peu plus haùt expliqué sur ce point (I). I1 suffit donc ici 'd'indiquer les types sociaux qui sont aujourd'hui admis. Voici les principaux. D'une part, l'histoire de l'occident montre qu'on y a connu, dans le monde grécoromain antique: $\mathrm{I}^{\circ}$ le type du genos et de la gens; $2^{\circ}$ celui de la cité $; 3^{\circ}$ célui de l'empire. Les deux. premiers ont été représentés par de multiples exemplaires; le troisième, au contraire, n'a eu qu'un modèle unique. Au temps de l'empiré romain, entrent en scène les tribus barbares, qui répondent à un type très différent. La destruction de l'empire par ces tribus, ou plutôt leur fúsion, amènent la constitution de formes sociales nouvelles. C'est ainsi qu'on voit naître : aux $\mathrm{v}^{\bullet}$ et $\mathrm{VI}^{\ominus}$ siècles, la villa et le domaine seigneurial; au $\mathrm{XI}^{\ominus}$ siècle, la commune; au $\mathrm{XV}^{\theta}$ siècle, 1'Etat moderne. - D'autre part, des études poursuivies sur des lieưx très différents ont singulièrement, de nos jours, élargi nos idées. Cette fois, ce n'est plus l'histoire européenne, c'est l'ethnographie, qui a éclairé la sociologie. On a étudié sur place les tribus arabes et berbères. On en a rapproché ce que 1'on savait, par la Bible, des tribus d'Israël. On a décrit la cité chinoise, le village annamite, la tribu mongole. Des formes sociales plus éloignées encore des nôtres ont apparu avec précision. Parmi elles, le type des tribus peaux-rouges s'est le premier constitué. Puis s'est dessiné le type

(I) Au chapitre XIV. 
des'tribus australiennes. On commence 'à fixer certains types de tribus du centre africain, celles des populations bantoues en particulier. - Pour caractériser les deux genres de travaux précédents, on pourrait citer les noms de deux auteurs éminents qui ont tenté d'en donner des synthèses: celui de Fustel de Coulanges pour l'antiquité et te moyen-âge (I), celui de Lewis H. Morgan pour les groupes extra-européens (2). Enfin, il convient de rappeler que, en utilisant ces diverses données, et en se servant aussi de çelles que fournissent l'archéologie préhistorique et même la zoologie, de hardis chercheurs essaient aujourd'hui de reconstituer le ou plutôt les types humains primordiaux, tentative qu'on ne doit pas dire prématurée, mais qui du moins est certainement assez loin' d'avoir abouti.

Venons-en maintenant aux lois dynamiques. Si l'on prend cet adjectif en son sens véritable, qui n'est pas celui qu'Auguste Comte a fait généralement accepter, l'expression désigne les lois qui régissent le jeu des forces sociales. On pourrait dire que ce sont les lois physiologiques des sociétés. La première de ces lois, celle qui domine toutes les autres, c'est la loi de moindre action ou

(1) Dans ses livres La cité antique et Histoire des institutions politiques de l'ancienne France.

(2) Ses travaux ont été résumés par Frédéric Engels dans ses Origine's do la famille, de la propriété privée et de l'Etat (traduction française par H. Ravé, I893). 
loi du moindre effort: Elle peut se formuler ainsi : "l'homme (ou le groupe) cherche à atteindre un résultat avec le moindre effort ou la moindre dépense possibles ", ou bien encore "il cherche, pour un effort ou une dépense donnés, à atteindre le résultat le plus élevé possible " (I). Cette loi a ceci de remarquable, qu'elle n'est pas seulement vraie dans le monde social, mais aussi dans le monde organique et déjà dans le monde physique. On reconnaît aisément en elle, ęn effet, un principe de la mécanique, le principe de moindre résistance. Ce qu'elle a de particulier dans le monde social, c'est qu'elle y prend un caractère psychologique. Ici, en effet, son application est consciente et vaulue. Elle y devient, suivant 1'expression du sociologue américain Lester Ward, "la loi d'économie ", laquelle préside, non seulement à l'activité proprement économique, mais à toutes les formes de l'activité humaine. - Après elle, on peut citer immédiatement les " lois de 1'imitation ") dégagées par Gabriel'Tarde. Nous rappelons que, pour celui-ci, le processus par lequel la société s'unifie, c'est l'imitation de ses membres les uns par les autres. Les lois de oe processus sont, suivant lui, au nombre de deux: l'imitation est d'abord unilatérale, puis

(I) Mais évidemment il ne faut pas dine que " il cherche, avec l'effort minimum, à obtenir le résultat maximum ", car cette formule ne comporte aucun élément de. détermination. 
réciproque (l'inférieur copie le supérieur, et plus tard est' aussi copié par lui); l'imitation va de l'intérieur à l'extérieur (on prend 1'esprit de son modèle avant de prendre sa forme). Sans vouloir les discuter ici à fond, il est juste de reconnaître que leur auteur a appelé l'attention sur des faits négligés avant lui, qu'il a tenté d'introduire quelque précision dans leur étude, qu'il a fait par là œuvre originale et suggestive.

Un sociologue belge que nous avons déjà cité,M. Guillaume De Greef, voit parfois rattacher à la théorie de Tarde son système propre, en raison d'une curieuse allitération ou consonnance. Ce qui fait le centre de ce nouveau systeme, c'est " la loi de limitation". Suivant celle-ci, toute action a forcément un champ limité, dans le domaine social. Pour ne prendre que ce dernier, les produits d'une industrie ne se diffusent pas partout, les dogmes d'une religion non plus; un code ne se fait admettre que dans un pays, ou tout au plus dans quelques-uns; les plus grands conquérants ne sauraient soumettre la terre entière. C'est la sauvegarde des autonomies, des initiatives, des diversités. Cette loi de limitation sert même en quelque sorte de contrepoids aux lois de l'imitatation, puisqu'elle empêche le jeu de celles-ci de rayonner indéfiniment. Nous n'hésitons pas à croire, pour notre part, qu'elle contient au moins autant de vérité que ces dernières.

Bornons-nous ici à une remarque. Ces diverses lois forment une sorte de transition entre les lois dynamiques et les autres lois sociales. La loi de 
limitation règle l'action des forces sociales en la réfrénant: elle a donc pour but d'assurer leur équilibre, et par là même elle a un caractère en partie statique. Les lois de l'imitation, à l'inverse, sont les lois d'une force transformatrice; ellesmêmes, dans leur propre énoncé, impliquent l'idée d'une succession de phases, d'une évolution ; elles ont déjà quelque chose de cinématique.

Nous sommes ainsi conduit aux lois proprement cinématiques. Ce sont, naturellement, des lois d'évolution. Elles se rangent elles-mêmes en deux groupes. Les unes s'attachent à l'évolution des structures; les autres, à cellès des fonctions.

C'est à ces dernières qu'appartient la plus connue des lois de la sociologie, celle qui constitua pour ainsi dire cette science tout entière à sa fondation, la loi des trois états d'Auguste Comte. On sait que, suivant elle, 1'humanité aurait passé, par trois grands états mentaux : état théologique (fétichisme, polythéisme, monothéisme), état métaphysique, état positif (âge de la spécialité, âge de la généralité). A chacune de ces formes de la mentalité correspond une forme de gouvernement: dans l'état théologique, le pouvoir appartient aux guerriers; dans l'état métaphysique, aux jurisconsultes; dans l'état positif, aux savants. A chacune correspond aussi une forme de l'industrie et une forme de l'art. - I1 y a certainement une grande hauteur de vues dans cette théorie, et l'on ne saurait contester qu'elle parte d'un principe exact: la marche de l'humanité vers un état 
où domine le savoir positif. Mais nous avons déjà dû faire nos réserves sur un autre de ses principes: la prépondérance du facteur intellectuel sur tous les autres facteurs sociaux (r). En outre, pour l'évolution du facteur intellectuel lui-même, nous ne voyons pas les choses tout à fait comme Auguste Comte. Depuis lui, la science a fait des progrès, et il faut aujourd'hui tenir compte d'éléments qu'il ne pouvait guère connaître: les recherches sur la proto-histoire et sur la pre-histoire. Pour lui, 1'état théologique était le règne de l'imagination; l'état métaphysique, celui du raisonnement ; l'état scientifique, celúi de l'expériénce. Mais les recherches auxquelles nous venons de faire allusion nous ont appris que le règne de l'imagination n'est pas le plus primitif de tous. Avant que cette faculté se développât, il y eut un 'temps où 1'humanité ne connaissait que la sensation brute, ou tout au plus que des perceptions coordonnées par une mémoire rudimentaire. A cette époque, les hommes étaient, suivant l'expression de M. S.-R. Steinmetz, professeur d'ethnographie à l'Université d'Amsterdam, "des matérialistes comme il n'y en a plus ". Ie développement de l'imagination a constitué un grand progrès par rapport à cet état primordial, et ainsi la théologie apparaît comme une forme déjà élevée de l'évolution intellectuelle. Si donc l'on tenait à. garder la division tripartite d'Auguste Comte, il

(I) Voir' chapitre XII. 
faudrait tout au moins y faire une importante correction. L'état métaphysique, dans lequel luimême ne voyait du reste qu'une transition, serait fondu avec ses deux voisins; l'état originaire, le plus long de tous, recevrait une place indépendante ; et l'on pourrait s'exprimer ainsi. L'humanité est partie d'une ère primitive, où toute pensée était étroitement concrète. Elle s'est ensuite élevée à l'abstraction, et elle a même failli s'y enfermer exclusivement. Elle revient de nos jours au concret, mais en bénéficiant des habitudes mentales dûes à l'abstraction. De la sensation, elle est passée à l'imagination et au raisonnement, et elle tend à arriver au plein savoir, qui utilise tous ces procédés à la fois ( $\mathrm{I}$ ).

Après le nom d'Auguste Comte, le plus grand de ceux dont la sociologie s'honore est le nom d'Herbert Spencer. Ce penseur a, lui aussi, formulé une loi générale qui concerne l'évolution des: fonctions sociales. Suivant elle, l'humanité passe progressivement du militarisme à 1'industrialismè. Au début, certains groupes vivent de leur tra-

(x) Mais d'ailleurs il ne paraît nullement indispensable de maintenir à tout prix le schéma d'Auguste Comte. Des écrivains de mérite en ont indiqué d'autres. C'est. ainsi que, à la loi des trois états, M. Louis Weber, dańs un livre récent sur Le rythme du progrès, propose de substituer une " loi des deux états ", fondée sur l'alternance habituelle des périodes où le progrès se fait dans l'ordre de la pensée et de celles où il s'opère dans l'ordre de l'activité matérielle. 
vail, mais d'autres subsistent à leur détriment, en les pillant. Plus tard, les choses se régularisent, et a la fin tous les groupes vivront de leur propre travail. L'activité prédatrice fait place partout, progressivement, à 1'activité créatrice. Nous estimons qu'il y a beaucoup à retenir de cette loi, malgré les cruels démentis que des événements récents lui donnèrent, montrant que le militarisme peut se développer avec l'industrialisme, et empêchant ainsi qu'elle soit prématurément généralisée. Mais nous ferons observer qu'elle n'est pas propre à Herbert Spencer. Déjà Comte avait insisté sur le fait que la prééminence sociale passe des guerriers aux industriels. Et Henri de SaintSimon, dont c'est un des titres de gloire, l'avait signalé avant lui. - D'autre part, un éminent historien du droit, Sir Henry Sumner Maine, avait présenté des vues analogues sous une forme plus proprement juridique. Il avait dit que l'évolution conduit l'humanité, du régime du statut, au régime du contrat. Le premier est celui où le gouvernement " statue " par voie d'autorité; le ' second, celui où les citoyens ne se sentent tenus que par leurs libres ententes. Mais le premier suppose le règne de la force armée; le second coïncide avec la pacifique organisation de l'industrie. On voit donc l'accord de la théorie de Maine avec celle de son compatriote Spencer. - Enfin, on peut noter que toutes deux ont inspiré de nos jours la formule d'un notoire économiste français, M. Yves Guyot, suivant laquelle, au cours de l'histoire, l'homme cesse d'exploiter son semblable pour exploiter la nature. 
Herbert Spencer a indiqué une seconde loi de l'évolution, non moins importante à ses yeux que la précédente. Celle-ci porte, non plus sur l'évolution des fonctions, mais sur celle des structures. Elle nous amène à nous expliquer sur les lois de ce dernier genre, qui sont peut-être, de toutes, celles qui pénètrent le plus au fond des choses. Cette seconde loi de Spencer se formule comme suit : l'évolution va, de l'hơmogène confus, à l'hétérogène coordonné. C'est, pour lui, une loi universelle de la nature. Dans le monde physique, elle caractérise la transformation des nébuleuses en planètes. Dans le monde vivant, elle préside au développement qui d'un embryon amorphe fait un organisme différencié. Dans le monde social, de même, elle conduit, de la horde primitive dont tous les membres se ressemblent et que rien'de précis ne dirige, à 1'Etat moderne où chaque individu a ses traits distinctifs, mais où mille liens définis l'unissent à ses divers associés. Peu de formules sont, croyons-nous, plus profondes que celle-là. Aucune ne " subsume " plus de faits. Nous observerons, en passant, qu'elle donne une solution originale à l'un des plus hauts problèmes que la philosophie se pose depuis Socrate et Platon: celui de la conciliation de l'un avec le multiple. Elle montre en effet, que dans tout être, l'unité et la multiplicité croissent à la fois, et elle porte par là à admettre que leur opposition n'est qu'apparente, puisqu'elles 'sont soumises à une loi de développement simultané.

Avant Spencer, un écrivain moins célèbre, peut- 
être mais éminent aussi, avait proposé une autre formule pour l'évolution des structures sociales. Nous voulons parler d'Adolphe Quetelet, contemporain d'Auguste Comte, et l'un des fondateurs de la statistique scientifique. Dans son remarquable Essai sur le développement. des facultés de l'homme (I), il expose que, par le fait de l'évolu-
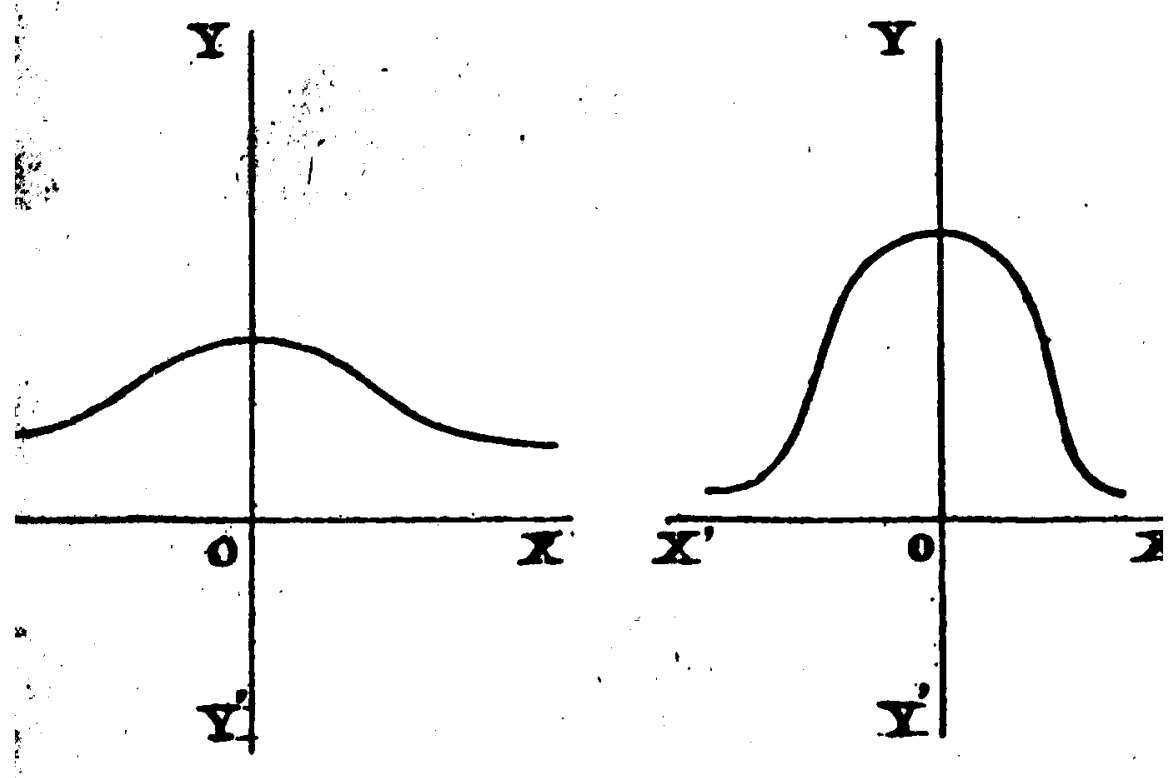

tion, les groupes humains s'unifient. Les écarts entre leurs membres se réduisent : écarts en force physique, en richesse, en savoir, en puissance. Le

(1) Bruxelles, 1835. Réédité en 1865 sous le titre de Physique sociale. 
noyau de la nation devient plus dense; les individus anormaux se font plus rares. Le type moyen, dans un groupe donné, s'affirme et prédomine. sans cesse davantage. - Quetelet donnait de cette. formule une illustration graphique saisissante. Il représentait les groupe par une " courbe en cloche " dont les individus moyens occupent le centre et dont les exceptions forment les extrémités. Avec le temps, disait,il, on voit le centre se relever et les extrémités se réduire et se rapprocherr (comme le montre la figure de la page I2 I). Ces vues semblent avoir eu quelque action sur la pensée de Cournot, et par elle sur celle de Tarde.

Un sociologue allemand contemporain, M. Ferdinand Tœennies, professeur de philosophie à 1'Université de Kiel, est l'auteur (I) d'une théorie que nous croyons devoir mentionner ici, comme: résumant des idées qui ont trouvé certain crédit chez quelques-uns de ses plus distingués compatriotes (2). Il sépare profondément la communauté et la société. Sous le nờn de communauté, il désigne les modes d'agrégation fermée : la famille domestique, l'Eglise, l'Etat. Sous le nom de société, il entend l'agrégation ouverte: la grande famille humaine, societas humani generis. L'évolution a consisté, d'après lui, dans le passage d'une communauté étroite à une communauté plus large, et dans la constitution progressive de la société pro-

(1) Dans son livre Gemeinschaft und Gesellschaft.

(2) Voir à cet égard Gaston Richard, La sociologie. 
prement dite. Combien le généreux auteur de ces vues doit regretter qu'en ce siècle même sa propre patrie aît entravé la venue du dernier stade évolutif par lui annoncé et souhaité!

S'il nous était permis, à notre tour ,de proposer une conception personnelle en ces délicates matierés, nous esquisserions la suivante. Deux mouvements simultanés et en apparence contraires, mais en réalité liés l'un à l'autre et complémentaires, se produisent dans les structures sociales. En vertu de l'un, les groupements s'amplifient. En vertu de l'autre, ils se divisent. Prenons le monde noderne à partir de l'invasion barbare. Les premières unités sociales sont très petites. Elles sont étroitement limitées au domaine de chaque chef. Peu à peu elles grandissent : par l'effet des luttes, des mariages, des héritages, un même chef devient Enaître de plusieurs domaines. Les comtés, les duchés se forment ainsi. Puis, la royauté englobe peu à peu toutes ces seigneuries : un véritable Etat se fonde. Ou plutôt il s'en fonde côte à côte une dizaine en Europe. Mais le mouvement ne s'arrête pas là. Le commerce devient, à partir du seizième siècle, international. Les idées scientifiques et philosophiques, comme précédemment les idées religieuses, franchissent les frontières. Les codes des divers pays se modèlent les uns sur les autres. Les mœurs tendent à s'unifier. Les lettres et les arts viennent, comme on l'a dit (I), "établir entre

(I) L'expression est de M. le recteur Gréard, dans un discours en Sorbonne aux étudiants étrangers. 
ceux qui les cultivent les liens d'une commune patrie ". Il se crée donc une unité européenne, ce qu'Auguste Comte appelait la république occidentale. Et comme ses principes rayonnent sur l'Amérique, puis de proche en proche sur les autres continents, on voit poindre l'unité humaine. Sans doute, il y a des reculs possibles, il y en a, hélas! de réels à l'heure présente, comme il y en eut un considérable dans l'antiquité, lorsque l'empire romain, produit d'une évolution du même genre, fut détruit par les invasions barbares. Mais enfin le mouvement est lancé et tout porte à croire qu'il ne s'arrêtera plus, qu'il triomphera des résistances et que son ouvre un jour se parfera. Seulement, en même temps que lui, on en aperçoit un autre tout différent. A l'intérieur des grandes nations qui se constituent, des divisions s'établissent. Des groupements très divers se créent, sur des principes nouveaux, suivant la profession, suivant la classe, suivant les " affinités électives ", sources de mille associations privées'de tout genre. Ainsi, à mesure que la société générale grandit, elle se fragmente en une multitude de sociétés particulières (I). Ce processus pourrait se traduire graphiquement par une série de figures représentant les stades successifs de la société. Dans la première, il y aurait une quantité de petits cercles indépendants, représentantt

(I) On trouverait d'ailleurs une loi analogue pour la croissance des organismes particuliers. 
chacun une société fermée (un domaine seigneurial). A la suivante, leur nombre se réduirait par leur fusion en des sociétés plus grandes (les comtés). A la troisième, il n'y en aurait plus que quelques-uns (les Etats). Mais déjà, dans la seconde, chaque cercle social verrait apparaître à son intérieur quelques petits cercles subordonnés, et le nombre de ceux-ci deviendrait très considérable dans la dernière. - En somme donc, l'évolution des structures sociales se résume, à nos yeux, en deux courants: l'un, qui diminue le nombre des groupements, et qui par conséquent unifie ; l'autre qui augmente ce nombre, et par conséquent diversifie Il n'y a là aucune contradiction : car les unités que le second courant fait naître ne sont pas du même ordre que celles que le premier élimine. On peut donc dire, cette fois encore, que l'unité et la multiplicité se sont accrues simultanément.

Voila; en somme, quelques-unes des lois que la sociologie a proposées. On ne saurait dire que toutes soient établies, ni même qu'aucune le soit entièrement. Mais toutes ont des faits à leur base, toutes semblent avoir une portée assez générale. Quoiqư'elles ne forment point jusqu'à présent un corps très cohérent, elles ne sont point opposées les unes aux autres. En les considérant, peutêtre voudra-t-on bien penser que, si la sociologie n'est pas faite, elle est du moins en voie de s'édifier. 


\section{TRÓISIÈME PARTIE}

\section{ATTACHES DE LA SOCIOLOGIE}

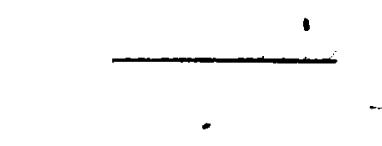

\section{CHAPITRE XVI}

RAPPORTS DE LA SOCIOLOGIE AVEC LA COSMOLOGIE ET LA BIOLOGIE.

Nous venons de voir en quoi consiste l'œuvre propre de la sociologie. Cela nous permet de cher-. cher quels sont ses rapports avec les disciplines voisines. Et cette investigation elle-même préciséra encore la nature des études sociologiques.

Il faut d'abord se demander quelles relations. la sociologie soutient avec les autres sciences génés 
rales qui furent constituées avant élle : nous voulons dire la cosmologie et la biologie.

I. - Le concept de cosmologie a quelque chosé d'assez peu précis. On peut, croyons-nous, désigner sous ce nom les théories d'ensemble relatives à la nature inorganique. Les vues les plus félevées sur la constitution et les mouvements des êtres matériels constituent cette étude. Elle est donc la synthèse des principes fondamentaux de la mécanique, de l'astronomie, de la physique et de la chimie.

De cette définition, on conclura immédiatement que la sociologie devra assez peu à une semblable science. Lês sociétés sont, en effet, trop loin des corps bruts pour que la considération de ceux-ci puisse apprendre grand chose sur celles-là. De nos jours pourtant, on a quelquefois méconnu cette vérité, éviđente a priori. On a essayé de constituer une mécanique sociale purement déductive. Les tentatives faites en ce sens, par des auteurs qui ne manquaient pourtant ni de savoir ni de talent - tels que Léon Winiarski en Suisse, Spiridion Haret en Roumanie et M. A. Portuondo y Barcelo en Espagne (I) - ont visiblement échoué. Ces

(1) Léon Winiarski, Deux théories d'équilibre économique, 1896 ; Quelques rectifications au sujet des essais récents de sociologie pure, I90o ; Le matérialisme historique et la mécanique sociale, I902. Sp. C. Haret, Mécanique sociale, 1910. A. Portuondo y Barcelo, Apuntes sobre mecanica social, rg12. 
auteurs ne sont arrivés, tout au plus, qu'à donner des expressions curieuses de faits ou de principes très connus. Rien de nouveau n'a pu être découvert par cette méthode. Et même la plupart des grands problèmes sociologiques n'ont pu être mis en équations. La complexité des facteurs qui y intervient, et leur incessante mobilité, rendaient la tâche impossible (I).

Est-ce à dire toutefois que la mécanique n'ait rien à enseigner à la sociologie? Nous ne saurions le croire. Au contraire, nous avons montré précédemment (2), en complétant et en rectifiant une vue d'Auguste Comte, que la division de la' mécanique peut être suivie dans les études sociales. - De même, nous admettons vountiers que l'astronomie, la physique et la chimie fournissent certaines' données de fait à la sociologie. Ainsi Comte a eu raison de dire que la vie sociale serait tout autre, si la longueur de l'année ou celle du jour venaient à changer. Pareillement, les lois. physico-chimiques créent à notre existence 'des' limites ou lui donnent des appuis que le sociologue ne peut ignorer. Mais c'est. tout. Quant à. l'utilisation des forces cosmiques par tel ou tel art

(I.) Il est à noter que M. Vilfredo Pareto, 1'un des: adeptes les plus autorisés de la méthode mathématique en économie politique, n'a pas tenté de l'introduire en: sociologie, lorsqu'il a abordé cette dernière science. Voir son Traité de sociologie générale, volume I, traduction. française, I9I7 ; volume II, traduction française,' 19 Ig.

(2) Dans notre chapitre $X$. 
social - par exemple, l'emploi des minéraux divers ou de la "houille blanche " par les industries - elle ne relève plus de la sociologie proprement dite.

II. - La biologie est plus voisine de la sociologie, puisque les phénomènes sociaux sont la vie externe des individus, dont les phénomènes organiques sont la vie intérieure. Le sọciologue aura donc besoin d'une initiation biologique. Mais ici encore, il faut se garder soigneusement de toute zxagération. La sociologie ne saurait être un simple prolongement de la biologie. Car d'abord, on peut remarquer que le concept de biologie, comme celui de cosmologie, n'est pas d'une précision extrême. On en est frappé, si l'on examine les ouvrages consacrés à cette science, tels que le Traité de biologie de F. Le Dantec ou les volumes de L'Année biologique dirigée par feu le professeur Yves Delage. Sous le nom de biologie, ces savants auteurs entendent des notions qui ne relèvent exclusivement, ni de la zoologie, ni de la botanique, ni de la protistologie ou microtiologie, ni de la paléontologie, mais qui les dominent toutes ( $\mathrm{r}$ ). Et sans doute, c'est une tentative

(I) Voici notamment les principales rubriques de L'Année biologique : la cellule ; la reproduction ; l'ontogénèse ; la tératogénèse ; la corrélation (des organes et des fonctions) ; le sexe ; la mort ; la morphologie générale et la chimie biologique ; la physiologie générale ; l'hérédité ; la variation ; l'origine des espèces et leurs caractè- 
intéressante que de fixer ces notions, mais pour beaucoup d'entre ellès on est extrêmement loin d'y être arrivé. Nous pouvons prendre comme exemple celle de la sexualité, qui est l'une des plus fondamentales. On ne sait nettement jusqu'à présent, ni sous quelles influences le sexe se détermine, ni même pourquoi la différence des sexes est apparue chez les êtres vivants (I). Cela n'impli-, que pourtant point que la biologie, quelque rudimentaire que soit son état actuel, ne puisśe rendre déjà de vrais services à la sociologie. Il est certain au contraire que Lamarck, Darwin et leurs émules. ont établi des principes rdont le sociologue, tout comme le naturaliste, peut faire son profit. Les notions d'adaptation, d'hérédité, de sélection, sont devenues primordiales, pour le premiey comme pour le second. Il n'est pas nécessairt. pour s'en convaincre, d'adopter la théorie de l'organisme social. Lors même qu'on se refuserait tout à fait à admettre que la société est un organisme, une chose resterait certaine : c'est qu'elle est un assemblage d'organismes, et que 'dès lors les lois qui régissent ces derniers ont leur répercussion sur elle. Aussi le sociologue ne, peut-i1

res ; la distribution géographique des êtres ; le système nerveux et les fonctions mentales.

(I) Voir le livre de M. Caullery, professeur de zoologie à la Sorbonne, sur Les problèmes de la sexualité, 1913, et notre propre ouvrage intitulé La sexualité dans les nais. sances françaises, I9I2. 
ignorer ces lois, et doit-il avoir fait une sérieuse etude des sciences organiques.

Mais ici il est à noter qu'une certaine réciprocité existe. Si la connaissánce de la biologie est utile au sociologue, celle de la sociologie l'est aussi au biologiste. Avant qu'il y eût une sociologie et même une biologie très développées, la plus avancée des sciences sociales, l'économie politique, avait inspiré d'éminents naturalistes. Henri Milne-Edwards, dans son Traité de physiologie, pour faire comprendre le fonctionnement de l'organisme humain, déclare proposer le terme de "division physiologique du travail ", sur le modèle de l'expression, courante chez les économistes, de "division du travail ". Charles Darwin lui-même, exposant ses vues célèbres sur la concurrence vitale ou lutte pour la vie, écrit dans l'Origine des espèces (I) que son propre système est " une généralisation de la loi de Malthus, appliquée au règne organique tout entier ". Les histologistes contemporains qui ont décrit la phagocytose, disent, dans leur interprétation de ce curieux phénomène, qu'il constitue une police de l'organisme, le moyen de défense que celui-ci emploie contre ses fauteurs de troubles intérieurs. On voit par là comment les sciences supérieures, après s'être inspirées des sciences inférieures (2),

(1) Chapitre III, $\$$ III.

(2) Nous ne voulons pas dire évidemment, que la sociologie soit plus noble ou plus avancée que la biologie, mais 
les peuvent à leur tour guider, et comment, entre ces deux séries de sciences, l'imitation, - suivant la formule de Tarde, quoiqu'en un sens opposé, après avoir été unilatérale tend à devenir réciproque.

seulement qu'elle s'occupe d'êtres plus complexes que ceux dont traite la biologie, donc d'êtres supérieurs. Nous employons simplement ici une expression abrégée qu'Au- : guste Comte a fait recevoir. 


\section{CHAPITRE XVII}

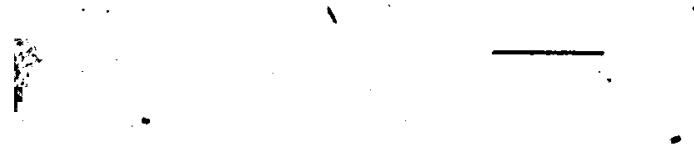

RAPPORTS DE LA SOCIOLOGIE AVEC LA PSYCHOLOGIE.

Dans son tableau hiérarchique des sciences fondamentales, Auguste Comte ne faisait aucune place à la psychologie. Il aimait peu celle-ci, à cause de la tournure que lui avait donnée l'école éclectique. I1 soutenait qu'elle se divisait réellement en deux parties, l'une rentrant dans la biologie ( la physiologie cérébrale), l'autre se rattachant à la sociologie et dominée par elle.

De nos jours, les esprits les plus positifs n'hésitent plus à voir dans la psychologie une science indépendante. Ils la placent tout naturellement -entre la biologie et la sociologie. Mais sur les rapports qu'elle a avec cette dernière, règne la plus Grande incertitude. Une série de propositions discordantes ont été émises à ce sujet. Ce qui complique la question, c'est qu'on y fait généralement intervenir un terme de plus, celui de "' psychologie collective " en l'opposant à celui de " psychologie individuelle ", mais sans définir suffisamment l'un 


\section{34}

ATTACHES DE LA SOCIOLOGIE

et l'autre. $\dot{A}$ notre sens, 1'emploi de ces deux móts est en effet nécessaire, mais ils doivent être pris en une acception différente de celle qu'on leúr attribue ordinairement. Nous allons essayer de la fixer tout d'abord.

La psychologie, nous semble-t-il,' peut porter ses investigations sur trois objets, nettement distincts par leur extension et par leur compréhension à la fois.

$I^{\circ}$ On peut faire la psychologie commune du genre humain, décrire les facultés qui se trouvent chez tous les hommes. C'est cette sorte de psychologie qui est couramment professée en France dans l'enseignement secondaire. C'est elle qu'aujourd'hui on nomme le plus souvent psychologie individuelle, pour la distinguer de la psychologie collective. Mais il nous paraît évident que cette appellation est tout à fait mal choisie. Car il n'y. a rien de moins individuel qu'une telle étude. Loin de porter sur l'individu, elle traite au contraire de ce qui est commun à tous les individus, ; de ce qui est identique chez eux tous. I1 serait donc logique de 1'appeler la psychologie générale ou psychologie humaine.

$2^{\circ}$ On peut, à l'intérieur du genre humain, en isoler une portion pour étudier sa mentalité propre: par exemple une nation, une ville, une profession, une classe, une association. Dans chacun des individus qui la composent, on trouvera simultanément des traits qui lui sont communs avec tous les hommes sans exception, et d'autres qui lui sont communs seulement avec les indi- 
vidus formant ce même groupè. Les premiers sont déjà connus par la psychologie humaine. Les seconds forment l'objet de cette nouvelle psychologie 'qu'on voit ici intervenir. On est convenu de l'appeler la psychologie collective. Bien que le mot ne soit pas très heureux, nous l'accepterons parce qu'il est déjà reçu pour désigner un ensemble de travaux intéressants, dont on peut voir dans l'Histoire de la littérature anglaise de Taine 1'un des meilleurs modèles.

$3^{\circ}$ Mais dans l'intérieur de ce même groupe, on trouve encore, en chaque individu, autre chose. A côté des traits qu'il partage avec tous les hommes, et de ceux qui le rapprochent des autres membres du groupe, il y a ceux qui n'appartiennent qu'à lui seul. Ces derniers constituent ses caractères originaux, sa différence propre. Ce sont eux que les biographies mettent le plus volontiers en évidence. Sans doute, par quelque côté, toute propriété mentale d'un homme le rapproche d'un de ses semblables. Mais la combinaison de ces propriétés est quelque chose d'original, d'unique. Et c'est à son étude que pourrait proprement s'appliquer le mot de psychologie individuelle.

Nous distinguons donc, en somme, trois sortes de psychologie: celle du genre humain, ou psychologie générale ; celle des groupes, ou psychologie collective; celle des personnalités, ou psychologie individuelle. I1 est évident que ces trois objets sont ici rangés dans leur ordre d'extension décroissante. Il est non moins clair qu'ils le sont, en même temps, dans leur ordre de compréhen- 
sion croissante. Car s'arrêter à la psychologie humaine, c'est forcément ne voir et ne décrire que des généralités abstraites (I). Envisager les caractères des différents groupes, c'est déjà faire œuvre plus complexe et plus concrète. Tenir compte des différences individuelles, c'est aller jusqu'au bout dans cette dernière voie.

Cela posé, il devient assez aisé de comprèndre les rapports de la sociologie avec la psychologie, ou plutôt avec les trois sortes de psychologie. La psychologie générale est celle qui confine le plus directement à la biologie. C'est celle qui a le moins de relations avec la sociologie. Au contraire, la psychologie collective implique essentiellement la notion de société. Elle ne peut se construire que par l'usage constant de données sociologiques. La mentalité d'une nation, par exemple, ne se comprend que si l'on connaît la constitution de ce groupe aux points de vue démique, ethnique, économique, domestique, juridique, politique, etc. Et réciproquement ces diverses questions s'éclairent par la connaissance de la mentalité collective. Enfin, la mentalité individuelle étant faite d'éléments sociaux diversement combinés, il faut, pour

(I) On essaie d'éviter cet inconvénient, dans l'enseignement secondaire, en décrivant les facultés humaines telles qu'elles se trouvent chez les hommes de notre pays et de notre temps, et chez les plus cultivés de tous. On fait ainsi appel aux deux dernières sortes de psychologie. Mais on se' met en dehors de la règle de la psychologie générale. 
l'étudier, connaître au préalable l'organisation sociale. On pourrait résumer tout cela en disant que, dans.l'ordre logique des sciences, la psychologie générale se place avant la sociologie (entre elle et la biólogie), que lá psychologie collective se place sur le même rang qu'elle, et que la psychologie individuelle se place après elle. Peut-être serait-ce, au fond, l'idée d'Auguste Comte, éclairée à la lumière des études postérieures à son œuvre.

- Les choses étant ainsi précisées, on est, croyonsnous, mieux en mesure de juger les thèses opposées qui.ont été émises sur les rapports entre la sociologie et la psychologie. Suivant Tarde, la sociologie sort tout entière de la psychologie; elle n'est, à vrai dire, qu'une psychologie agrandie, une inter-psychologie. Suivant Durkheim, à l'inverse, la sociologie peut et doit se constituer en dehors de la psychologie, ou du moins de la psycholdgie "individuelle ", ce mot étant pris à la fois dans son acception usuelle et dans son acception logique; et e'est au contraire la psychologie qui réclame pour s'établir des secours de la sociologie. La vérité est plus complexe que ces formules d'un décevante simplicité. La sociologie a besoin de la psychologie générale ou humaine. Car on ne peut rien comprendre à un édifice social quelconque si l'on ignore la constitution mentale de l'homme. Elle a même besoin de la psychologie collective, car les caractères particuliers d'une société déterminée ne sont intelligibles que pour qui connaît la tournure d'esprit de șes membres. Enfin, 
quelquefois elle utilise jusqu'à la psychologie individuelle, au sens vrai de ce dernier terme. Car tel homme éminent a exercé sur les destins d'une. société, ou même de 1'humanité, une action considérable, et il importe de connaître jusqu'aux moindres particularités de sa vie mentale. Tels sont, en bref, les services que les trois sortes de psychologie peuvent rendre à la sociologie, et ils sont de premier ordre.

Mais, réciproquement, la sociologie peut aussi leur en rendre de fort sérieux, à toutes les trois. Nous l'avons montré déjà :' la psychologie vraiment individuelle ne se constitue, normalement, qu'avec des données sociales, et la psychologiè collective s'organise en même temps que la socio-y logie, par un incessant échange de services réciproque. Mais même la psychologie générale utilise la sociologie. 'Parmi les facultés mentales communes à tous les hommes, les plus hautes se sont, nous ne saurions dire créées, mais du moins singulièrement perfectionnées, par l'effet de la vie sociale. Déjà, M. Izoulet avait écrit : " la raison est fille de la cité " (I) et cette formule a eu dú succès, bien qu'elle ne fût chez son auteur appuyée que de preuves sommaires. Durkheim semble. 1'avoir reprise en quelque sorte à son compte. I1 a voulu montrer, par des faits positifs, comment certaines facultés s'élaborent au sein du groupejo Par exemple, il a indiqué comment l'aptitude $\dot{x}$

(1) La cité moderne: 
classer s'y est constituée. Les primitifs ont d'abord divisé les membres de leur propre société en diverses séries. Puis ils ont étendu les notions tirées de là à tous les êtres vivants. Les animaux, les plantes, même les corps bruts, se sont ainsi vu diviser en classes parallèles à celles des humains. L'origine de la notion de genre est donc toute sociale (I): M. Lévay-Bruhl, se plaçant dans le même ordre d'idées, a recherché la raison de la parenté que les primitifs imaginent entre leurs clans et les espèces vivantes, et qui est le principe du totémisme. I1 la trouve dans l'imprécision des frontières qui pour le sauvage séparent les êtres, dans le fait que pour celui-ci toutes choses participent jusqu'à un certain point à la nature les unes des autres. Il a été ainsi amené à formuler une " loi de participation " régissant la mentalité des

(1) Voir E. Durkheim, Les formes elémentaires de la vie religieuse : le système totémique en Australie, 1912 ; notamment chapitre III : le système cosmologique du totem et la notion de genre. - E. Durkheim et M. Mauss, De quelques formes primitives de classification, dans L`Année sociologique, tome VI. Ces auteurs ont essayé de montrer que les divisions de l'espace sont tirées des divisions de la tribu. - Il est à remarquer que, avant eux, M. Henri Bergson avait indiqué la relation de la notion d'espace avec l'existence collective, en 1888 , dans son Essai sur les données immédiates de la conscience (ainsi, page ro4 : " l'intuition d'un espace homogène est déjà un acheminement à la vie sociale 
primitifs ( $\mathrm{I}$ ). - Il y a, dans les efforts de ces auteurs, quelque chose d'intéressant. Car tout ce qui nous renseigne sur les progrès de l'esprit humain vaut la peine d'être relevé, et il n'est guère douteux que ces' progrès se soient faits en grande partie par une application de l'effort mental aux choses de la vie sociale. Mais, si cette application a perfectionné des facultés, en a-t-elle créé? Sur ce point, la lumière n'est pas faite. La thèse de Durkheim ne se verra pas aisément accepter. Beaucoup y résisteront, dont les uns voudront maintenir l'indépendance de la psychologie par l'innéité des facultés humaines, dont les autres affirmeront les droits de la biologie et voudront faire remonter l'origine de ces facultés à l'évolution animale. Le débat n'aura quelques chances d'être tranché, et même la question ne pourra être posée avec une complète précision, que lorsque la proto-histoire et la préhistoire seront beaucoup plus avancées qu'elles ne le sont aujourd'hui.

En tous cas, une chose est établie. C'est que la psychologie et la sociologie peuvent se rendre, à tous les stades, des bons offices fort importants. Il est donc certain qu'il faut se garder d'opposer ces deux sciences, et que l'intérêt de toutes deux est d'observer l'une envers l'autre les règles d'une courtoise et confraternelle collaboration.

(1) L. Lévy-Bruhl, Les fonctions mentales dans les sociétés inférieures, rg1o. 
RAPPORTS DE LA SOCIOLOGIE AVEC LES DIVERSES SCIENCES SOCIALES.

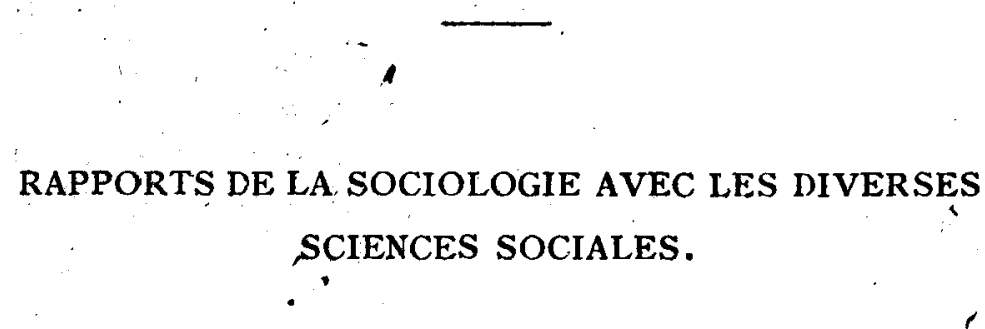

Quelles sont les relations de 1a sociologie avec les sciences sociales particulières? Nous avons déjà abordé cette question dans la première partie de ce travail, où nous avons conclu que la sociologie est la philosophie de ces sciences. Ici nous n'avons donc à y revenir que pour régler certains poințs, que les idées formulées dans la seconde partie vont nous permettre d'élucider.

Aux sciences sociales particulières, comme à la psychologie, la sociologie a beaucoup à demander et beaucoup à donner à la fois. Qu'elle ait beaucoup à leur demander, la chose ne peut pas être un seul instant douteuse. Nous avons vu, en effet, qu'elle constitue, à proprement parler, leur synthèse. Il est donc clair que c'est d'elles que lui viennent tous ses éléments. Elle est, par suite, incessamment leur tributaire. On ne peut guère 
concevoir, semble-t-il, un lien de dépendance plus étroit que celui qui l'attache à elles.

Mais ce lien est réciproque. Si la sociologie sort des sciences sociales, elle les domine. Elle est à la fois leur base et leur sommet. Elle est le point d'où elles partent toutes dans des voies divergeantes, et aussi le point où elles convergent quand elles ont achevé leurs œuvres propres. Essayons de montrer les services qu'elle leur rend à ce double sujet.

Au premier point de vue, elle leur sert d'introduction. C'est donc à elle à fixer la tâche qui revient à chacune de ces sciences dans l'œuvre commune de l'investigation sociale. Elle à dès lors à délimiter leurs objets et à tracer leurs méthodes. Et voici ce qu'elle peut indiquer à cet égard.

Les objets des sciences sociales sont, en quelque sorte, parallèles aux objets des sciences organiques. Prenons l'étude des êtres vivants, telle qu'on la fait aujourd'hui. Elle peut se traiter de deux façons. D'une part, on peut examiner tour à tour les diverses espèces, chacune avec tous ses caractères structuraux et fonctionnels. On constitue alors 1'histoire naturelle descriptive, comprenant la zoologie et la botanique. D'autre part, on peut envisager isolément les diverses structures et les diverses fonctions, chacune dans toutes les espèces qui la présentent. On constitue alors l'anatomie comparée et la physiologie comparée. Les deux procédés se valent. Ils sont indispensables l'un et l'autre. Ce sont les mêmes éléments qu'ils envisa- 
gent, mais en les disposant dans un ordre invèrse. Et il y a intérêt pour la science à ce que ces deux modes d'enquête soient poursuivis parallèlement.

Eh bien! il en est tout à fait de même pour les sociétés. Ici aussi, on peut et on doit envisager: d'abord, les diverses sociétés, chacune avec toutes ses particularités; ensuite, les divers éléments et les divers ordres de faits sociaux, chacun dans tơus les groupes qui le présentent. La première de ces deux études, comparable à l'histoire naturelle descriptive, c'est l'histoire humaine descriptive, l'histoire des nations et des autres groupes 'húmains, en un mot ce qu'on est habitué à entendre appeler l'histoire tout court. La seconde, c'est l'ensemble des sciences sociales comparatives, dont chacune limite son étude à l'un des éléments ou à l'un des ordres de faits dont nous avons donné précédemment l'énumération. Voici donc la liste de ces sciences. Examinent les structures sociales: la géographie sociale, ou géographie humaine, étude du milieu physique dans ses rapports avec les sociétés; la démographie, étude de la population (de son chiffre, de sa répartition, des phénomènes qui l'affectent); l'ethnographie, étude des races; enfin cette science très jeune qu'on a parfois' appelée la morphologie sociale, à laquelle revient l'étude des groupements compris dans les nations (professions, classes, associations). Examinent, d'autre part, les phénomènes sociaux: la science économique; la science des mours (dont l'étude des faits familiaux est une branche); la science des religions; l'histoire des 
sciënces, des lettres et des arts ; la science juridique ou histoire du droit; la science politique. Tel est, ce nous 'semble, le tableau général des sciences sociales.

C'est la sociologie qui le dresse, et qui seule a qualité pour le dresser, n'étant pas l'une d'entre ellés, mais se superposant à leur ensemble. Ce sera donc à elle; en cas de contestation, à leur assigner leurs frontières respectives. - 'Et de même, c'est elle qui élucide leur méthode. C'est à elle, notamment, à affirmer que ces sciences ne sont pas des arts, qu'elles' ne tendent pas à l'application, mais seulement à la connaissance. C'est ì elle encore à les garder contre les séductions de la méthode dialectique, pour les astreindre à suivre les règles de la minutieuse observation et de la prudente induction. Nous avons consacré plús haut, à ce dernier point, un chapitre spécíal (I). Sans doute en tout cela, comme d'ailleurs en ce qui concerne les objets des sciences sociales, elle ne fait que dégager ce qui résultait du développement spontané de ces sciences. Mais elle le fait. avec plus d'autorité qu'aucune d'elles ne pourrait le faire, attendu qu'elle ne se confine dans le champ étroit d'aucune, : et rayonne au contraire sur les domaines d'elles toutes. En utilisant leurs données, elle est amenée à juger celles-ci et par là à apprécier les procédés qui ont permis de les atteindre. Elle note dans ses synthèses ceux de

(I) Le chapitre XIV. 
ces procédés qui lui ont paru particulièrement dignes d'approbation. Et ainsi elle conseille implicitement à tous les chercheurs de s'en inspirer éventuellement ( 1 ).

Reste encore le second point de vue. La sociologie n'est pas setilement le point de départ des sciences sociales, elle en est aussi le point d'arrivee. A l'instant, nous venons de l'apercevoir en ce qui concerne les méthodes, puisque celles-ci, bien que théoriquement indiquées au début de chaque science, ne peuvent pratiquement être fixées qu'à son terme. Mais nous le voyons surtout en ce qui regarde le contenu même des sciences sociales particulières. Les lois qui les résument, les conclusions auxquelles elles aboutissent, sont transmises par elles à la sociologie, dont c'est la tâche de les synthétiser. Tâche éminemment délicate, mais aussi éminemment fructueuse! Faire tenir en quelques phrases simples un résumé de l'évolution humaine et de ses principaux aspects, quel but plus séduisạnt un penseur pourrait-il se proposer? Nous avons vu précédemment quelques-unes des tentatives déjà faites à cet égard (2). Nous avons dû conclure qu'aucune n'a définitivement réussi, mais que la voie demeure ouverte

(1) Par exemple, en signalant les méthodes d'investigation précise en usage dans la science économique (la otatistique, la monographie, l'enquête), la sociologie en amènera peut-étre la diffusion dans les autres sciences sociales particulières.

(2) Dans notre chapitre XV. works 
et que les chances de succès sont sérieuses. C'est quand ce succès aura été obtenu, qu'on pourra pleinement juger de ce nouveau service rendu par la sociologie aux sciences sociales. En condensant leurs découvertes en des formules lapidaires qui se graveront dans 1'esprit de tous, elle leur aura donné la consécration la plus haute que la science puisse espérer. L'ambition du savant n'est-elle pas de léguer à l'humanité une parcelle de vérité dont chacun fasse consciemment son profit? 


\section{CHAPITRE XIX}

RAPPORTS DE LA SOCIOLOGIE AVEC LES ARTS SOCIAUX.

Les arts sociaux sont, d'après une définition que nous avons antérieurement justifiée, des constructions théoriques tendant à l'amélioration du monde social. Ce ne sont point des sciences. Toutefois, la distinction de la science et de l'art ne s'est pas encore fait recevoir et comprendre partout, et l'on est assez habitué à entendre qualifier sciences des arts comme la morale et la politique.

En est-il ici des arts sociaux comme des sciences sociales? On pourrait croire $a$ priori, par analogie avec ce qui se passe pour celles-ci, que la sociologie a avec ceux-là des rapports de deux sortes, qu'elle leur emprunte et qu'elle leur donne. Mais ici, son apport à elle nous paraît de beaucoup supérieur à la contribution qu'elle peut recevoir. D'abord, aux arts sociaux, la sociologie rend le service de bien définir leurs objets et leurs méthodes. Elle précise la notion d'art, en la dis- 
tinguant nettement de la notion de science. Elle pose les limites réciproques des divers arts sociaux, en même temps que les limites de chacun d'eux et de la science sociale à laquelle il correspond. En principe, on peut penser qu'il doit $y$ avoir, pour chaque objet social, une science qui. 1'étudie et un art qui vise à le perfectionner. Toutefois, ce parallélisme n'est pas absolú. Il existe pleinement pour les sciences de fonctions. A la science économique répond 1'art économique; à la science des mours, la morale; à l'histoire des lettres et des arts, l'esthétique (envisagée comme règle du goût, dans ses diverses branches); à la science des religions, la dogmatique religieuse; à la science juridique ou histoire du droit, le droìt luimême, nous voulons dire ici la technique du législateur et du juge; à la science politique, la politique proprement dite. Mais déjà, pour les sciences de structures, des lacunes apparaissent dans la série des arts corrélatifs. A la démographie répondent l'eugénique (I) et l'hygiène sociale; une partie de la morphologie sociale répond la civique. A la géographie sociale répondent, si l'on veut, tous les arts utiles qui ont pour fin la bonne exploitation de notre planète; la corrélation pourtant devient ici assez vague. Elle dispa-

(I) L'eugénique donne des préceptes pour la bonne genèse des individus; la civique en donne pour la bonne construction des villes. Leurs noms sont venus d'Angleterre (eugenics, civies) et le second n'est pas encore tres répandu en France. 
raît presque, lorsque des sciences sociales còmparatives on passe à la science sociale descriptive, l'histoire proprement dite, l'histoire des nations. Comment trouver un art propre à l'améliotation de chaque nation? Ce qu'on peut seulement dire, c'est que chacun des arts sociaux précités tient compte du milieu national dans lequel ses préceptes devront s'appliquer, c'est qu'il nuance ceux-ci suivant ce milieu. Mais c'est tout. I1 ne faut donc pas vouloir pousser à l'extrême le parallélisme des'arts sociaux et des sciences sociales. Si imparfait qu'il soit, pourtant, il conserve quelque portée, et c'est la sociologie qui l'a reconnu et établi.

De même encore, c'est elle qui relève les procédés des arts sociaux, en les distinguant des procédés des sciences sociales. Nous avons indiqué précédemment que les arts ont une méthode descendante, par opposition avec la méthode ascendante des sciences. Nous avons montré ainsi que ceux-là font de la déduction et de l'expérimentation un plus grand usage que celles-ci (r). Ce sont des points sur lesquels il n'y a donc pas lieu de revenir ici.

Il convient, en outre, de noter le rôle que la sociologie peut jouer, au profit des adeptes des divers arts sociaux, comme propédeutique générale. Chacun de ceux qui veut cultiver un de ces arts doit évidemment connaître la science cor-

(I) Chapitre XIV. 
respondante. I1 serait bon qu'il connût aussi les autres sciences sociales. Mais on ne saurait l'exiger : on craindrait que ce ne fût trop lui demander. Peut-être du moins a-t-on le droit de vouloir qu'il en prenne une vue d'ensemble. Ce sera dans la sociologie, synthèse de toutes ces sciences, qu'il. pourra le mieux le faire. Un exemple va préciser. notre pénsée. Beaucoup de nos contemporains émettent des théories politiques, dans les Parlements, dans les livres, dans les journaux. Ils font donc de 1'art politique. Nécessairement, ils ont dû apprendre quelque peu de science politique: il faut qu'ils connaissent bien la constitution actuelle de leur pays, et qu'ils aient certaines notions sur 1'histoire de cette constitution et sur 1'organisation publique des principaux pays étrangers. Certes, il serait fort désirable qu'ils fussent, en outre, au courant du détail des sciences démographique, économique, morale, juridique: leur patrie en bénéficierait, et aussi leur propre carrière. Mais, s'ils ne le sont pas, ce qui pourra avoir plus d'une excuse, il y aurait avantage à ce que du moins ils eussent une notion sommaire de ce que sont ${ }^{\prime}$ les sociétés en général, leurs structures, leurs fonctions, leur évolution. Si la sociologie le leur a appris, les théories qu'ils formuleront ne pourront qu'être plus sages.

De tous les arts sociaux, il en est un sur lequel notre attention doit ici s'arrêter un moment : c'est l'art moral. C'est lui qu'on nomme d'ordinaire " la morale " sans plus. Quel est donc son lien avec la sociologie? Dans un livre assez récent, 
qui résụme tout un courant d'idées, on a soutenu que la sociologie était destructive de la morale (I). L'auteur de ce livre entendait sous le nom de sociologie le système de Durkheim, et sous le nom de morale celle de Saint-Thomas. Il n'est guère contestable, en effet, que les points de vue de ces deux penseurs ne soient pas les mêmes. Mais il y a quelque étroitesse à vouloir réduire toute morale au thomisme, et toute sociologie à une doctrine particulière. Il est assez peu exact en fait que les idées de Durkheim soient contraires aux principes de la morale traditionnelle. Et surtout, il est inexact que celles de tous les sociologues le soient. Au contraire, nous estimons qu'aucune étude ne peut, mieux que la sociologie, inspirer le respect de cette morale. Car, ce qui résulte des investigations sociologiques, c'est que la morale n'est pas l'invention de quelques individus, qui pourraient être des hommes de génie, mais qui pourraient être aussi des imposteurs; qu'elle est au contraire l'invention collective de l'humanité ; qu'elle est le produit d'une élaboration continue, poursuivie dans tous les temps et dans tous les lieux; qu'elle condense l'expérience de l'élite ; qu'elle formule les principes par la pratique desquels cette élite s'est naturellement " séligée " ; qu'étant le meilleur résumé du passé, elle est le guide le plus sûr de l'avenir. Peut-il y avoir pour

(x) Simon Deploige, Le conflit de la morale et de la sociologie, Louvain, 1911 . 
la morale traditionnelle, aux yeux d'un homme éclairé par la science, des titres plus beaux que ceux que la sociologie vient ainsi lui conférer?

Quant aux services que les arts sociaux rendent à la sociologie, ils ne nous apparaissent guere. Sans doute, pour bien connaître un état social, il faut savoir les théories de réforme qui y sont écla. ses et la suite qu'elles ont comportée. Mais en s'en informant, on ne fait œuvre, à vrai dire, que de science. - Sans doute encore, d'un autre côté, il serait intéressant, pour le sociologue, d'avoir été lui-même mêlé à l'action sociale: cela l'aiderait: à mieux comprendre les phénomènes qu'il doit interpréter. Mais la pratique n'est pas l'art: celuici est la théorie, celle-là est la réalisation. - En somme, on ne voit pas très bien à quoi servirait au sociologue d'avoir lui-même construit des théo. ries d'action, ce qui est le propre de l'art social. On peut supposer que de semblables theories? émises par lui, seraient supérieures à celles qui sortent de 1'imagination d'un fantaisiste quelconque. Seulement, ce serait encore là un service rendu par la sociologie à 1'art social, et non par celui-ci à celle-là. Nous ne croyons pas que la sociologie d'Auguste Comte ait beaucoup gagné à ses projets de réorganisation "sociocratique ", ni la sociologie d'Herbert Spencer à ses proposi, tion économiques d'un individualisme si intran. sigeant. Nous devons donc, au moins jusqu'à plus ample information, demeurer quelque peu sceptiques sur la valeur du concours à attendre par la sociologie des arts sociaux. 


\section{CHAPITRE XX}

RAPPORTS DE LA SOCIOLOGIE AVEC LA PHILOSOPHIE.

La dernière question à envisager par nous sera celle des relations qui unissent la sociologie à la philosophie. I1 est bien _évident que, par.cette expression, c'est la philosophie générale que nous entendons désigner. Au reste, en ce qui concerne la psychologie et la morale, que l'on considère souvent comme des fractions de la philosophie, nous avonis déjà examiné précédemment les liens qu'elles présentent avec la sociologie (I).

Mais la philosophia générale elle-même peut s'entendre en des sens différents. Ou du moins, il y a des façons assez variées d'en poser et d'en résoudre less problèmes capitaux. Chacune a sa raison d'être; chacune mérite l'attention et l'intérêt, Suivant celle qu'on adoptera, la question que nous nous posons ici changera d'aspect.

(I) Chapitre XVII es XIX. 
I. - L'une des manières de définir la philosophie, et l'une des plus récentes, consiste à y voir une synthèse générale des sciences. C'est ainsi qu'Auguste Comte et qu'Herbert Spencer ont éla-. boré, l'un sa Philosophie positive, l'autre sa $P$ hi-' losophie synthétique. Dans une semblable conception, le rôle de la sociologie par rapport à la philosophie peut se définir d'une manière assez simple. La sociologie, en effet, est alors la partie la plus élevée de la philosophie. Car cette dernière est un édifice à trois étages : philosophie cosmique, philosophie organique, philosophie sot ciale, et le couronnement de l'édifíce est ce qui s'y remarque le plus. La sociologie devient ainsi le point d'aboutissement de toute la recherche philosophique. On peut dire à certains égards que celle-ci est faite entièrement pour la préparer et la rendre possible. La préoccupation sociologique finit même par devenir parfois un peu excessive chez les deux illustres auteurs que nous venons de nommer. Ainsi, pour Auguste Comte, la science n'a de valeur que par rapport à l'humanité. Le principe est certain. Mais en doit-on conclure que la science ne vaut que par son usage social (I)? Comte le faisait, et c'est pourquoi il condamnait comme vaines et inspirées par une curiosité fort oiseuse, les recherches n'ayant aucun trait à l'humanité. Ainsi il voulait que 1'astrono-

(I) Nous ne disons pas : son usage matériel, car Comte aurait tout à fait répugné à aller jusque-là. 
mie se bornât à l'étude du monde solaire, et lui interdisait l'examen de l'univers stellaire. Nous ne croyons pas qu'on puisse, en aucun cas, le suivre sur ce point. L'enquête sur les parties les plus reculées 'de l'univers peut avoir la même valeur scientifique que l'investigation sur des domaines très proches. Elle satisfait, au moins autant que cèlle-ci, les plus nobles aspirations de l'esprit. Elle sert, au moins autant, à sa formation. Et nul ne peut dire qu'elle sera toujours dénuée de portée pratique (I). La scienče doit donc être cultivée pour elle-même, et non pour l'usage immédiat que la société en peut faire. - Il y a encore une autre manière vicieuse d'affirmer la suprématłe du point de vue sociologique. C'est celle qui consiste à introduire sans cesse des notions sociologiques dans les sciences organiques et même cosmiques. Certes, le supérieur ne peut pas s'expliquer tout entier par l'inférieur, et ce serait une prétention inacceptable que célle qui tendrait à réduire le monde social au jeu des lois physicochimiques, ou même biologiques. Mais il ne faut

(r) Qui peut savoir, du rèste, ce qui aura un jour une application pratique? Auguste Comte lui-même, dans cet ordre d'idées, a appelé l'attention sur un fait remarquable. Les spéculations des géometres grecs sur les sections coniques ont permis de comprendre l'orbite des planètes. Or l'observation des astres sert à guider le navigateur. Ces spéculations toute théoriques à leur origine ont donc ultérieurement reçu les applications les plus fécondes. 
pas, inversement, vouloir retrouver tout le superieur chez l'inférleur, et il serait très vain de parler sans mesure le langage social sur des questions organiques ou cosmiques. On n'arriverait ainsi qu'à embrouiller ces quéstions, et ce serait une fausse lumiere qu'on prétendrait y projeter. La sociologie doit se garder de semblables intru, sions, qui risqueralent fort de compromettre son autorité, admit-on celle des conceptions philosophiques qui lui fait la plus large place.

II. - A cette conception s'en oppose, d'une certaine manière, une autre. Lés partisans de celle-cl accepteralent bien encore de définir la philosophie en fonction de la science. Seulement, au lieu de placer la philosophie après la science, ils la placeraient avant. Nous n'entendons pas prendre ces expressions dans un sens hiérarchique, mais dans un sens logique. Pour cette seconde écolé, la phllosophie est l'étude des conditions a priori de la connaissance, des principes qui président à la formation et au développement de toute pensée. Elle est, pour reprendre ane expression de Kant, l'illustre initiateur de cette conception, une critique gênérale du savoir.

Que peut, aux yeux d'une philosophie de ee genre, valoir la sociologie? Sans parler des diffijcưltés particulières que rencontre sa tâche, elle est nécessairement soumise aux mêmes conditions reștrietives que toutes les autres sciences. Elle est obligé de se plier aux cadres de l'esprit humain, c'est-à-dire aux formes de la sensibilité et aux 
principes de la raison. La matière extérieure (dont la société est ici un élément) n'y entre qu'en sé déformant. Nous ne saisissons que le phénomène. La réalité dernière, le fond des êtres, le noumène, nous échappe. Ainsi la sociologie n'est, comme chaque science, qu'une science des apparences.

A cette critique, si fondée qu'elle soit, les sociologues pourraient répondre par une contre-critique. Oui, diraient-ils, notre étude est subordonnée aux conditions générales de la connaïssance. Mais les principales de ces conditions, d'où donc dérivent-elles? Cet élément que l'esprit met dans toute connaissance, et qu'il ajoute aux choses extérieures, ce n'est pas un principe premier et par suite inexplicable. C'est un trésor qui s'est lentement formé par l'accumulation d'apports successifs. Les uns sont d'ordre biologique, les autres sont d'ordre social. Déjà Spencer avait indiqué que les principes de la raison, quoique innés aujourd'hui chez l'individu, peuvent avoir été acquis, au cours de l'évolution, par l'effort cohérent et continu de l'espece. Ies sociologues contemporains poursuivent leurs recherches dans la même voie. Ils s'efforcent de montrer que notre constitution mentale actuelle dérive, en grande partie, de la vie collective, par l'attention que les hommes ont da porter aux conditions de l'organisation de leur groupe. A vrai dire, la preuve ne nous en paraît pas entièrement faite; et le fût-elle, la totalité de cette constitution mentale ne serait pas expliquée. On ne peut toutefois contester 
qu'il y ait quelque chose d'intéressant dans cette sentative, et qu'elle mérite d'être poursuivie. Si elle devait un jour aboutir, il faudrait reconnâ̂tre que dans les conditions de la connaissance intervient largement un facteur social, que dès lors la critique du savoir ne peut se faire a priori, et que, dans une forte mesure, elle relève de la sociologie elle-même.

III. - Il existe encore une autre conception de la philosophie générale. C'est la plus ancienne de toutes, celle qui peut se réclamer des penseurs les plus nombreux et les plus célèbres, la conception métaphysique. Quelques divergences que présentent les systèmes inspirés par elle, il est une. idée directrice qui leur est commune, et elle nous paraît la suivante. En dehors et au-dessus des réalités qui se comptent et se pèsent, il en est d'autres qui échappent à ces procédés de. mesūre et qui n'en ont que plus de prix. Le domaine des forces matérielles n'est pas tout: il existe aussi celui des forces morales. Celles-ci ne peuvent être saisies, du moins intégralement, par la science; mais elles tombent sous les prises de la conscience. On ne sauraif les exprimer pleinement en termes quantitatifs ; mais elles comportent une énonciation qualitative, et celle-ci les juge en même temps qu'elle les fait comprendre. En un mot donc, avec la métaphysique, on ne se borne plus, comme dans la philosophie des sciences, à résumer l'expérience sensible, ou, comme dans la critique, à en montrer la portée limitée; on la 
dépasse véritablement, par l'élan de l'esprit et du cœur. Derrière la surface mobile des phénomènes, on entrevoit la substánce stable de l'être. Par delà le relatif, le contingent et le fini, on pressent l'absolu, le nécessaire et le divin.

Que vaut une semblable conception? Ce n'est pas ici le lieu de l'étudier. Nous avons seulement à rechercher, en ce qui nous concerne, l'attitude qui en face d'elle convient à la sociologie. Et à cet égard il nous semble qu'il y a une méprise à dissiper. Trop souvent on a admis qu'il existe un antagonisme entre la métaphysique et la science, et, rangeant la sociologie parmi les sciences, on en a conclu qu'elle est une des ennemies de la métaphysique. Cette posture agressive a bien été prise, en effet, par certains sociologues. Mais nous n'hésitons pas à dire qu'elle nous paraît déplacée. De part et d'autre, en effet, des concessions sont souhaitables et faciles. Nous nous plaisons à reconnaître que, du côté des métaphysiciens, on les fait aujourd'hui volontiers. La métaphysique n'aspire plus - si même elle y a jamais aspiré - à remplacer la science. Elle demande simplement à garder son domaine propre, qui commence où celui de la science finit. De plus en plus elle professe de la sympathie pour le savoir positif, de plus en plus elle en utilise les données. C'est justice de constater combien, depuis un quart de siècle, elle s'est assimilé de données provenant des sciences mathématiques, physiques, biologiques, ou de la psychologie expérimentale et comparée. Croit-on qu'elle ne soit pas disposée 
à en faire autant vis-à-vis de la sociologie ? Le jour est proche, sans doute, où elle cherchera résolument à s'appuyer sur des bases sociales. Car, de tous les domaines naturels̀, le domaine social est celui qui peut fournir les données les plus directement utilisables par elle. C'est dans celuici en effet que l'on voit à l'œuvre les forces morales, que l'on trouve agissant l'esprit et le cœur. Or la sociologie constate les effets de ces causes que la métaphysique, elle, pense saisir dans leur essence même. La première livre donc à la seconde des faits que cette dernière peut immé. diatement interpréter. L'une voịt du dehors ce que l'autre espère pénétrer jusqu'au dedans. Celle-ci a donc tout avantage à s'appuyer sur celle-là, et nous pensons qu'elle le ferait très volontiers, 'si d'une part les résultats de la sociologie étaient mieux établis et plus précis, et si d'autre part les sociologues s'y prêtaient eux-mêmes de meilleure grâce.

Nous nous tournerons donc maintenant vers les sociologues et nous leur dirons : la métaphysique a droit à tous les égards, de votre point de vue. Elle résume, en effet, les idées générales qu'une société se fait de l'être, de la nature, de la vie, de la destinée, et en même temps les idées généreuses qui peuvent inspirer la conduite et l'action individuelles ou collectives. Connaître la métaphysique d'un peuple, c'est connaître le fond de son âme. Un pays et une époque sont éminemment caractérisés par leurs vues sur les problèmes derniers. Ainsi 1'histoire de la pensée philosophique 
est le centre de l'histoire de l'esprit humain, c'està-dire de l'histoire humaine presque entière. Elle est donc exceptionnellement instructive pour une science qui veut donner la synthèse de l'évolution de notre espèce. - Ce n'est point tout. Non seulement 1'histoire de la métaphysique, mais ses doctrines actuelles, sont de la plus haute portée sociale. Par son affirmation du facteur idéal, elle empêche l'esprit de se trop complaire dans les trivialités du réel. Elle revendique une place, à côté de la matière, pour la force ; à côté de l'étendue, pour le mouvement; à côté du corps, pour l'esprit ; à côté du visible et du palpable, pour ce que la raison seule atteint. Elle élève l'intelligence vers ce qui est grand, abstrait, désintéressé, intangible. Elle est, par là, une source intarissable d'énergie virile; à laquelle s'alimentent de nos jours et viendront sans doute longtemps s'alimenter ceux auxquels il appartient de présider à la marche ascendante de la civilisation.

Ainsi sociologie et métaphysique ne s'opposent point. Chacune doit respecter l'autre. Chacune peut trouver en l'autre un concours. Dans le champ des études philosophiques, il y a place côte à côte pour toutes les bonnes volontés. 



\title{
TABLE DES MATIERES
}

\author{
PREMIÉRE PARTIE \\ NATURE DE LA SOCIOLOGIE
}

Chapitres

Pages

ler.... Position de la question . . . . . . I

II .... La sociologie n'est pas un art . . . . 6

III... La sociologie n'est' pas une science spéciale . . . . . . . . . . . 12

IV.... En quel sens la sociologie est la science . générale des sociétés . . . . . . . $\quad 17$

V.... La sociologie est la philosophie des sciences sociales particulières . . . . 23

\section{SECONDE PARTIE}

\section{CONTENU DE LA SOCIOLOGIE}

VI... La société et les relations sociales. . . $\quad 27$

VII... Le fait social fondamental . . . . . 33

VIII .. Réalité de la société . . . . . . . 40

IX... Contrat social ou organisme social . $\quad 48$

X.... Division de la sociologie. . . . . . 56

XI.... Les éléments sociaux . . . . . . . 64

XII... La vie sociale . . . . . . . . . 74 


\section{TROISIĖME PARTIE}

\section{ATTACHES DE LA SOCIOLOGIE}

XVI .. Rapports de la sociologie avec la cosmologie et la bìologie. . . . . . . . $\quad{ }^{126}$

XVII.. Rapports de la sociologie avec la psychologie . . . . . . . . . . . ${ }_{1} 33$

XVIII. Rapports de la sociologie avec les diverses sciences sociales . . . . . . $14 \mathrm{I}$

XIX .. Rapports de la sociologie avec les arts sociaux . . . . . . . . . 147

XX... Rapports de la sociologie avec la phiflosophie . . . . . . . . . ${ }_{153}$ 